\title{
NUCLEOSYNTHESIS IN CHANDRASEKHAR MASS MODELS FOR TYPE IA SUPERNOVAE AND CONSTRAINTS ON PROGENITOR SYSTEMS AND BURNING FRONT PROPAGATION
}

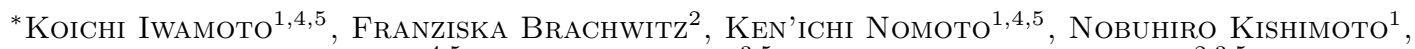 \\ HIDEYUKI UMEDA $^{4,5}$, W. RAPHAEL HIX ${ }^{3,5}$, FrIEDRICH-K. THIELEMANN ${ }^{2,3,5}$ \\ ${ }^{1}$ Department of Astronomy, University of Tokyo, Tokyo 113-0033, Japan \\ 2 Department of Physics and Astronomy, Universitty of Basel, CH-4056 Basel, Switzerland \\ 3 Oak Ridge National Laboratory, Oak Ridge, TN 37831-4576, USA \\ ${ }^{4}$ Research Center for the Early Universe, School of Science, University of Tokyo, Tokyo 113-0033, Japan \\ ${ }^{5}$ Institute for Theoretical Physics, Santa Barbara, CA 93106-4030, USA \\ Published in the Astrophysical Journal Supplement Series, 1999, v.125, pp.439-462
}

\begin{abstract}
The major uncertainties involved in the Chandrasekhar mass models for Type Ia supernovae (SNe Ia) are related to the companion star of their accreting white dwarf progenitor (which determines the accretion rate and consequently the carbon ignition density) and the flame speed after the carbon ignition. We calculate explosive nucleosynthesis in relatively slow deflagrations with a variety of deflagration speeds and ignition densities to put new constraints on the above key quantities. The abundance of the Fegroup, in particular of neutron-rich species like ${ }^{48} \mathrm{Ca},{ }^{50} \mathrm{Ti},{ }^{54} \mathrm{Cr},{ }^{54,58} \mathrm{Fe}$, and ${ }^{58} \mathrm{Ni}$, is highly sensitive to the electron captures taking place in the central layers. The yields obtained from such a slow central deflagration, and from a fast deflagration or delayed detonation in the outer layers, are combined and put to comparison with solar isotopic abundances. To avoid excessively large ratios of ${ }^{54} \mathrm{Cr} /{ }^{56} \mathrm{Fe}$ and ${ }^{50} \mathrm{Ti} /{ }^{56} \mathrm{Fe}$, the central density of the "average" white dwarf progenitor at ignition should be as low as $\lesssim 2$ $\times 10^{9} \mathrm{~g} \mathrm{~cm}^{-3}$. To avoid the overproduction of ${ }^{58} \mathrm{Ni}$ and ${ }^{54} \mathrm{Fe}$, either the flame speed should not exceed a few $\%$ of the sound speed in the central low $Y_{e}$ layers, or the metallicity of the average progenitors has to be lower than solar. Such low central densities can be realized by a rapid accretion as fast as $\dot{M} \gtrsim 1 \times 10^{-7} \mathrm{M}_{\odot} \mathrm{yr}^{-1}$. In order to reproduce the solar abundance of ${ }^{48} \mathrm{Ca}$, one also needs progenitor systems that undergo ignition at higher densities. Even the smallest laminar flame speeds after the low-density ignitions would not produce sufficient amount of this isotope. We also found that the total amount of ${ }^{56} \mathrm{Ni}$, the $\mathrm{Si}-\mathrm{Ca} / \mathrm{Fe}$ ratio, and the abundance of some elements like $\mathrm{Mn}$ and $\mathrm{Cr}$ (originating from incomplete Si-burning), depend on the density of the deflagration-detonation transition in delayed detonations. Our nucleosynthesis results favor transition densities slightly below $2.2 \times 10^{7} \mathrm{~g} \mathrm{~cm}^{-3}$.
\end{abstract}

\section{INTRODUCTION}

There are strong observational and theoretical indications that Type Ia supernovae (SNe Ia) are thermonuclear explosions of accreting white dwarfs (e.g., Wheeler et al. 1995; Nomoto, Iwamoto \& Kishimoto 1997; Branch 1998). Theoretically, both the Chandrasekhar mass white dwarf models and sub-Chandrasekhar mass models have been considered (see, e.g., Arnett 1996; Nomoto et al. 1994, 1996a, 1997b, 1997c; Canal, Ruiz-Lapuente, \& Isern 1997 for reviews of recent progress). Though these white dwarf models can account for the basic observational features of SNe Ia, the exact binary evolution that leads to SNe Ia has not been identified yet. Various evolutionary scenarios have been proposed, which include (1) a double degenerate scenario, i.e., the merging of two $\mathrm{C}+\mathrm{O}$ white dwarfs in a binary system with a combined mass exceeding the Chandrasekhar mass limit (e.g., Iben \& Tutukov 1984; Webbink 1984 ) and (2) a single degenerate scenario, i.e., accretion of hydrogen or helium via mass transfer from a binary companion at a relatively high rate (e.g., Nomoto 1982a). In the case of helium accretion at low rates, He detonates at the base of the accreted layer before the system reaches the Chandrasekhar mass (Nomoto 1982b; Woosley \& Weaver 1986, 1994a; Livne \& Arnett 1995). Currently, the issues of the Chandrasekhar mass versus sub-Chandrasekhar mass models and the double degenerate versus single degenerate scenarios are still debated (see, e.g., Renzini 1996 and Branch et al. 1995 for recent reviews), but they are being confronted with an increasing number of observational constraints.

The observational search for the double degenerate scenario led to the discovery of a few binary white dwarfs systems, but with combined mass being smaller than the Chandrasekhar mass (Renzini 1996). Theoretically, it has been suggested that the merging of double white dwarf systems leads to accretion-induced collapse rather than to SNe Ia (Nomoto \& Iben 1985; Saio \& Nomoto 1985, 1998). The Chandrasekhar versus sub-Chandrasekhar mass issue has recently experienced some progress. Photometric and spectroscopic features of $\mathrm{SNe}$ Ia in early phases clearly indicate that Chandrasekhar mass models give a much more consistent picture than the sub-Chandrasekhar mass models of helium detonations (e.g., Höflich \& Khokhlov 1996; Nugent et al. 1997). This leaves us with the most likely progenitor system, a single degenerate system with hydrogen accretion from the companion star, leading to a Chandrasekhar-mass white dwarf. However, the Chandrasekhar mass model W7 (Nomoto, Thielemann, \& Yokoi 1984; Thielemann, Nomoto, \& Yokoi 1986), widely used in galactic chemical evolution calculations, may require im-

*Present Address: Department of Physics, Nihon University, Tokyo 101-8308, Japan 
provements in terms of the Fe-group composition because it predicts significantly higher ${ }^{58} \mathrm{Ni} /{ }^{56} \mathrm{Fe}$ ratios than solar. The direct determination of $\mathrm{Ni}$ abundances in late time SN Ia spectra is therefore important (Ruiz-Lapuente 1997; Liu, Jeffery, \& Schultz 1997; Mazzali et al. 1998). The recent findings of supersoft X-ray sources, being potential progenitors of SN Ia events with high accretion rates, causing ignition at low densities (van den Heuvel et al. 1992; Rappaport, Di Stefano, \& Smith 1994; Di Stefano et al. 1997), leave hope for Chandrasekhar-mass models, which meet all these requirements, also for late time spectra.

The presupernova evolution of an accreting white dwarf depends on the accretion rate $\dot{M}$, the composition of the material transferred from the companion star, and the initial mass of the white dwarf (e.g., Nomoto 1982a; Nomoto \& Kondo 1991). Chandrasekhar mass white dwarfs can be obtained with a relatively high mass transfer rate of hydrogen of the order $\dot{M} \approx 4 \times 10^{-8}-10^{-5} \mathrm{M}_{\odot} \mathrm{yr}^{-1}$. At $\dot{M}>4 \times 10^{-6} \mathrm{M}_{\odot} \mathrm{yr}^{-1}$, the accreting white dwarf blows off a strong wind, which reduces $\dot{M}$ to an effective accretion rate below $10^{-6} M_{\odot} \mathrm{yr}^{-1}$ (Hachisu, Kato, \& Nomoto 1996, 1999a). This avoids the formation of an extended envelope in the accreting white dwarf. (Nomoto , Nariai, \& Sugimoto 1979). At such rates hydrogen and helium burn steadily or with weak flashes, leading to a white dwarf with a growing $\mathrm{C}+\mathrm{O}$ mass.

For the Chandrasekhar mass white dwarf model, carbon ignition in the central region leads to a thermonuclear runway. The ignition density depends on the stellar structure as a function of previous accretion history. High accretion rates lead to higher central temperatures, i.e. favoring lower ignition densities. A flame front then propagates at a subsonic speed $v_{\text {def }}$ as a deflagration wave owing to heat transport across the front (Nomoto et al. 1984). The major and yet not fully solved questions are related to the propagation of the burning front. Timmes \& Woosley (1992) have analyzed the propagation speed of laminar burning fronts in one dimension as a function of density and fuel composition. However, the propagation in three dimensions is influenced by instabilities that can enhance the effective radial flame speed beyond its laminar value.

The flame front is subject to various types of instabilities, namely, thermal instabilities (Bychkov \& Liberman 1995a), the Landau-Darrius (L-D) instability (Landau \& Lifshitz 1987), the Rayleigh-Taylor (R-T) instability, and the Kevin-Helmholtz (K-H) instability (Niemeyer, Woosley, \& Hillebrandt 1996). The turbulent burning regime associated with the R-T bubbles on global scales has been studied (Livne 1993; Arnett \& Livne 1994a; Khokhlov 1995; Niemeyer \& Hillebrandt 1995a; Niemeyer et al. 1996), but there remain many uncertainties, related partially to numerical resolution but also to the role and spectrum of turbulent length scales (Hillebrandt \& Niemeyer 1997). With the present uncertainties, it is essential to perform parameterized sets of calculations that explore the possible range of effective radial flame speeds.

In the deflagration wave, electron captures enhance the neutron excess. The amount of electron capture depends on both $v_{\text {def }}$ (influencing the time duration of matter at high temperatures, and with it the availability of free protons for electron capture and the high-energy tail of the electron energy distribution) and the central density of the white dwarf $\rho_{9}=\rho_{\mathrm{c}} / 10^{9} \mathrm{~g} \mathrm{~cm}^{-3}$ (increasing the electron chemical potential). The resultant nucleosynthesis in slow deflagrations (see, e.g., Khokhlov 1991b) has some distinct features compared with faster deflagrations like W7 (Nomoto et al. 1984; Thielemann et al. 1986), thus providing important constraints on these two parameters. The constraint on the central density is equivalent to a constraint on the accretion rate, as discussed above. After an initial deflagration in the central layers, the deflagration is accelerated as in W7, or assumed to turn into a detonation at lower densities, as in the delayed detonation models (Khokhlov 1991a; Woosley \& Weaver 1994a). For the latter, the uncertain transition density $\rho_{\text {tr }}$ would result in a variety of total masses of ${ }^{56} \mathrm{Ni}$ and expansion velocities of the outer layers.

To obtain constraints on the three parameters $\left(\rho_{\text {ign }}\right.$, $v_{\text {def }}$, and $\rho_{\text {tr }}$ ), we performed explosive nucleosynthesis calculations for slow deflagrations followed by a delayed detonation or a fast deflagration. These calculations assumed spherical symmetry and therefore might not be fully adequate for a realistic and consistent approach, but we expect that they lead at least to some clues how abundance features relate to the spherical average of these quantities in realistic models. Initially slower deflagrations cause an earlier expansion of the outer layers with respect to the arrival of the burning front (as information of the central ignition propagates with sound speed; Nomoto, Sugimoto, \& Neo 1976) and lead to low densities for the outer deflagration and detonation layers. In such a case, even a detonation does not lead to a pure Fe-group composition, as expected in central detonations, and intermediate mass elements from $\mathrm{Si}$ to $\mathrm{Ca}$ are produced at a wide range of expansion velocities. Therefore, if the deflagrationdetonation transition (DDT) density is well tuned, delayed detonations can meet these observational requirements as well as fast deflagration (Kirshner et al. 1993). Compared with the earlier delayed detonation models by Khokhlov (1991b), we adopt a larger and more detailed nuclear reaction network that alos includes electron screening. In comparison to the fast deflagration models by Woosley (1997b), we use initial models with lower central densities and smaller flame speeds, to concentrate on our main aim, which is to find the "average" SN Ia conditions responsible for their nucleosynthesis contribution to galactic evolution, i.e., especially the Fe-group composition.

From the very early days of explosive nucleosynthesis calculations, when no direct connection to astrophysical sites was possible yet, it was noticed (Trimble 1975) that the solar Fe-group composition could be reproduced with a superposition of matter from explosive Si burning with about $90 \%$ originating from a $Y_{e}=0.499$ source and $10 \%$ from a $Y_{e}=0.46$ source. As it has been shown that SN II ejecta with $Y_{e}<0.498$ could cause serious problems in comparison with observations (Thielemann, Nomoto, \& Hashimoto 1996), SNe Ia have to be identified with this second source and the appropriate conditions which lead to the best agreement with solar abundances. For this reason we present detailed yields of delayed detonation models as well as fast deflagration models and compare them with solar abundances for a number of "training sets" of ignition densities, flame speeds, and DDT densities.

These nucleosynthesis constraints can provide clues to 
the explosion mechanism (i.e., the speed of the burning front) and the ignition density (i.e., the accretion rate from the binary companion) for the "average" or dominating SN Ia contributions during galactic chemical evolution. This is the purpose of the present paper. We have to be aware that there are some systematic variations that manifest themselves in light curves (i.e., the brighter one is slower; Phillips et al. 1990; Hamuy et al. 1995) and might lead to a variation in nucleosynthesis as well (Höflich \& Khokhlov 1996, Höflich, Wheeler \& Thielemann 1998). It is further of importance to explore metallicity effects, which might have an influence on the evolution of the progenitor systems, with respect to the initial mass function (IMF) and composition of white dwarfs as well as the binary accretion history (e.g., Yoshii, Tsujimoto, \& Nomoto 1996; Höflich et al. 1998; Umeda et al. 1999; Höflich et al. 1999). Only the latter will clarify whether the nature of SNe Ia at high redshifts is the same as for nearby SNe Ia, which enters the determination of the cosmological parameters $H_{0}$ and $q_{0}$ (e.g., Branch \& Tammann 1992; Riess, Press, \& Kirshner 1995; Riess et al. 1999; Perlmutter et al. 1997, 1999). Observable spectral features could possibly help to identify the metallicity due to slight changes in nucleosynthesis (Höflich et al. 1998; Hatano et al. 1999; Lentz et al. 1999).

After a description of our model calculations in $\S 2$, including initial models, the hydrodynamic treatment, and a discussion of the input physics, we present in $\S 3$ detailed nucleosynthesis results from slow deflagrations and delayed detonations in comparison with the carbon deflagration model W7. In $\S 4$, the integrated abundances of SNe Ia models are combined with those of SNe II to compare with solar abundances. Finally, we discuss constraints on possible evolutionary scenarios and give conclusions in $\S 5$. Very preliminary accounts of the present investigations on nucleosynthesis in slow deflagrations and delayed detonations have been given in Nomoto et al. (1997c) and Thielemann et al. (1997).

\section{INITIAL MODELS, EXPLOSION HYDRODYNAMICS, AND INPUT PHYSICS}

\subsection{Initial Models}

We adopt two models with central densities of $\rho_{9}=1.37$ $(\mathrm{C})$ and $2.12(\mathrm{~W})$ at the onset of thermonuclear runaway, i.e., at the stage when the timescale of the temperature rise in the center becomes shorter than the dynamical timescale. Here $\mathrm{C}$ and $\mathrm{W}$ imply that these are the same models as calculated for $\mathrm{C} 6$ and $\mathrm{W} 7$, respectively (Nomoto et al. 1984). The initial white dwarfs of these models, before the onset of $\mathrm{H}$-accretion, have a mass of $M=1.0 \mathrm{M}_{\odot}$, a central temperature of $T_{c}=1.0 \times 10^{7} \mathrm{~K}$, and compositions of $X\left({ }^{12} \mathrm{C}\right)=0.475, X\left({ }^{16} \mathrm{O}\right)=0.50, X\left({ }^{22} \mathrm{Ne}\right)=$ 0.025 .

The outer layers of the mass grid extend to the steady hydrogen burning shell as an outer boundary. The temperature and density at the burning shell are determined from the boundary condition that the accreted matter is processed into helium with the mass accretion rate $\dot{M}$. These values increase from initally $8 \times 10^{7} \mathrm{~K}$ and $1 \times 10^{4} \mathrm{~g}$ $\mathrm{cm}^{-3}$ to $1 \times 10^{8} \mathrm{~K}$ and $1 \times 10^{6} \mathrm{~g} \mathrm{~cm}^{-3}$ at the point of central carbon ignition. The accretion rate for case $\mathrm{C}$ is due to steady and stable hydrogen burning correspond- ing to the $\mathrm{C}+\mathrm{O}$ core increase during an asymptotic giant branch evolution (see Nomoto 1982a). The rate for case W is kept constant up to the point of carbon ignition at the center. The exact values are given by equations (1) and (2), where $M$ indicates the mass of the accreting white dwarf.

$$
\begin{aligned}
\dot{M}(\mathrm{C}) & =8.5 \times 10^{-7}\left(M / \mathrm{M}_{\odot}-0.52\right) \mathrm{M}_{\odot} \mathrm{y}^{-1} \\
\dot{M}(\mathrm{~W}) & =4 \times 10^{-8} \mathrm{M}_{\odot} \mathrm{y}^{-1}
\end{aligned}
$$

During the accretion phase the white dwarf mass $M$ increases with time and the central temperature increases as a result of heat inflow from the H-burning shell as well as compressional heating. Cooling is due mostly to plasmon neutrino losses and neutrino bremsstrahlung. Cooling due to Urca shells and the convective Urca process is not taken into account. This has no effect on case $\mathrm{C}$, but could delay the ignition in case $\mathrm{W}$ to higher densities (e.g., Paczynski 1973; Iben 1982; Nomoto \& Iben 1985; Barkat \& Wheeler 1990). When the central density $\rho_{\mathrm{c}}$ reaches $1.5 \times 10^{9} \mathrm{~g}$ $\mathrm{cm}^{-3}$ (C) or $2.5 \times 10^{9} \mathrm{~g} \mathrm{~cm}^{-3}(\mathrm{~W})$, carbon is ignited in the center, where the nuclear energy generation rate exceeds the neutrino losses. When the central temperature increases owing to carbon ignition, a convective core develops. The convective energy transport is calculated in the framework of the time-dependent mixing length theory (Unno 1968). At $T_{\mathrm{c}} \sim 8 \times 10^{8} \mathrm{~K}$ convection can no longer transport energy in our model and the central region undergoes a thermonuclear runaway with $\rho_{\mathrm{c}}=1.37$ $\times 10^{9} \mathrm{~g} \mathrm{~cm}^{-3}$ (C) or $2.12 \times 10^{9} \mathrm{~g} \mathrm{~cm}^{-3}$ (W).

\subsection{Slow Deflagrations}

We know the absolute lower limit for the deflagration speed after central ignition from the one-dimensional analysis of laminar flame fronts (Timmes \& Woosley 1992), being close to $1 \%$ of the local sound speed $v_{s}$. On the other hand, any hydrodynamic instability can enhance the speed. Our parametrized "fast" deflagration studies, which reached $10 \%-30 \%$ of the sound speed and produced the model W7 (Nomoto et al. 1984; Thielemann et al. 1986), however, resulted in problematic Fe-group nucleosynthesis (see also discussion below). The flame speed found in multidimensional hydrodynamic simulations is still subject to large uncertainties, ranging from $v_{\text {def }} / v_{\mathrm{s}} \sim$ 0.015 (Niemeyer \& Hillebrandt 1995a) to $v_{\text {def }} / v_{\mathrm{s}} \sim 0.1$ (Niemeyer et al. 1996). Therefore, it is important to investigate how the nucleosynthesis outcome depends on the flame speed. In order to contrast our "fast" deflagration model W7, we study "slow" deflagrations here and choose the following parameter ranges: After the central thermonuclear runaway, we assume that a slow (S) deflagration propagates with speeds $v_{\text {def }} / v_{\mathrm{s}}=0.015$ (WS15, CS15), 0.03 (WS30, CS30), and 0.05 (CS50) and consider also extreme cases of fully and initially laminar flame fronts (WLAM, WSL). The location of the deflagration wave in radial mass coordinate $M_{r}$ and the changes in temperature and density are shown in Figure 1 i as a function of time. Behind the deflagration wave, the temperature rises quickly to values as high as $T=9 \times 10^{9} \mathrm{~K}$ and the material experiences nuclear statistical equilibrium (NSE). As 
the flame front propagates outward, the white dwarf expands slowly which reduces the central density $\rho_{\mathrm{c}}$. The decrease in $\rho_{\mathrm{c}}$ for these slow deflagrations is significantly slower than in W7.

\subsection{Transition from Deflagration to Detonation}

If the deflagration speed continues to be much slower than in W7, the white dwarf undergoes a large amplitude pulsation, as first found by Nomoto et al. (1976). In this pulsating deflagration model, the white dwarf expands and nuclear burning is quenched when the total energy of the star is still negative. In the following contraction more material burns, resulting in a positive total energy $E$. Eventually the white dwarf is completely disrupted. The model by Nomoto et al. (1976) resulted in $E=5 \times 10^{49}$ ergs and a ${ }^{56} \mathrm{Ni}$ mass of $M_{\mathrm{Ni}} \sim 0.15 \mathrm{M}_{\odot}$. Such a pulsating deflagration produces explosion energies too small to account for typical SNe Ia but might be responsible for rare events such as SN 1991bg.

In order to produce sufficient amounts of radioactive ${ }^{56} \mathrm{Ni}\left(\sim 0.6 M_{\odot}\right)$ to power SNe Ia light curves by a deflagration wave, the flame speed must be accelerated. The degree to which the flame speed is increased depends on the effect of R-T instabilities during the pulsation (Woosley 1997a). The deflagration might induce a detonation when reaching the low-density layers. In the delayed detonation model (Khokhlov 1991a; Woosley \& Weaver 1994b), the deflagration wave is assumed to be transformed into a detonation at a specific density during the first expansion phase. In the pulsating delayed detonation model (Khokhlov 1991b), the transition into a detonation is assumed to occur close to the maximum compression after recontraction, as a result of mixing.

Physical mechanisms by which such deflagration-todetonation transitions (DDTs) occur have been studied by Arnett \& Livne (1994b), Niemeyer \& Woosley (1997), Khokhlov, Oran, \& Wheeler (1997), and Niemeyer \& Kerstein (1997): (1) When a sufficiently shallow temperature gradient is formed in the fuel, a deflagration propagates as a result of successive spontaneous ignitions. Such an over-driven deflagration propagates supersonically. (2) If a sufficiently large amount of fuel has such a shallow temperature gradient, the deflagration may induce a detonation wave. The critical masses for the formation of a detonation are quite sensitive to the carbon mass fraction $X(\mathrm{C})$, e.g., $\sim 10^{-19} \quad \mathrm{M}_{\odot}$ and $\sim 10^{-14} \quad \mathrm{M}_{\odot} \quad$ at $\rho \sim 3 \times 10^{7} \mathrm{~g} \mathrm{~cm}^{-3}$ for $X(\mathrm{C})=1.0$ or 0.5 , respectively. (Niemeyer \& Woosley 1997). (3) Such a shallow temperature gradient region in the fuel may be formed if the fuel is efficiently heated by turbulent mixing with ashes. Such a mixing and heat exchange may occur when the turbulent velocity associated with the flame destroys the flame (Niemeyer \& Woosley 1997; Khokhlov et al. 1997). Note that whether the DDT occurs by this mechanism is controversial (Niemeyer 1999), and thus the exact density of the DDT is still debated (Niemeyer \& Kerstein 1997). Therefore, nucleosynthesis constraints on the DDT density are important to obtain.

Motivated by the discussion above we transform the slow deflagrations WS15 and CS15 artificially into detonations when the density ahead of the flame decreases to 3.0, 2.2, and $1.7 \times 10^{7} \mathrm{~g} \mathrm{~cm}^{-3}$ (DD3, DD2, and DD1, respectively, where 3,2 , and 1 indicate $\rho_{7}=\rho / 10^{7} \mathrm{~g} \mathrm{~cm}^{-1}$ at the DDT). Then the carbon detonation propagates through the layers with $\rho<10^{8} \mathrm{~g} \mathrm{~cm}^{-3}$. Figures 2 and 3 show the density distribution and expansion velocity after the passage of the slow deflagration and the subsequent delayed detonation. The explosion energy $E$ and the mass of synthesized ${ }^{56} \mathrm{Ni}$ of these WSDD/CSDD models as well as W7 and W70 are summarized in Table 1. Here W70 is the same hydrodynamical model as W7 except for the initial mass fractions of $X\left({ }^{22} \mathrm{Ne}\right)=0.0, X\left({ }^{12} \mathrm{C}\right)=0.50, X\left({ }^{16} \mathrm{O}\right)=$ 0.50 . This corresponds to zero initial metallicity because ${ }^{22} \mathrm{Ne}$ originates from the initial $\mathrm{CNO}$ elements.

The increase of the ${ }^{56} \mathrm{Ni}$ mass from W7 to W70 is due to the fact that the composition in W70 corresponds to a $Y_{e}=0.5$ or a proton/neutron ratio of 1, i.e., symmetric matter. In mass zones that are not affected by electron capture but which undergo complete Si burning, ${ }^{56} \mathrm{Ni}$ is then the dominant nucleus, without competition by more neutron-rich species. Detonations in lower density matter do not necessarily lead to complete Si burning. Therefore, the amount of ${ }^{56} \mathrm{Ni}$ is smallest in DD1 models and largest in DD3 models. The small difference between WS and CS DD-models is again a $Y_{e}$ effect. CS models have a smaller central density, which leads in the central regions to a smaller number of electron captures and larger $Y_{e}$ values, resulting again in more symmetric matter.

\subsection{Input Physics}

The methods for performing these calculations were the same as used in Shigeyama et al. (1992) and Yamaoka et al. (1992). We apply an implicit Lagrangian hydrodynamics code (Nomoto et al. 1984) for the slow deflagration and a Lagrangian PPM hydro code, as used in Shigeyama et al. (1992), for the detonation phase. Both codes use the same mass grid of 200 radial zones for the white dwarf. The nuclear reaction network and the reaction rate library utilized are the same as described in Thielemann et al. (1996), i.e., thermonuclear rates using the Hauser-Feshbach formalism (Thielemann, Arnould, \& Truran 1987), experimental charged particle rates from Caughlan \& Fowler (1988), neutron induced rates from Bao \& Käppeler (1987), and extensions towards the proton and neutron drip lines from van Wormer et al. (1994) and Rauscher et al. (1994) with Coulomb enhancement factors from Ichimaru (1993). Electron capture rates were adopted from Fuller, Fowler, \& Newman (1980, 1982, 1985). For nuclei beyond $A=60$, only ground state decay properties were used. We consulted the analysis of Aufderheide et al. (1994) so that the influence of a nucleus with a significant impact on $Y_{e}$ (either via decay or electron capture) was neglected in either of the conditions experienced in our calculations. It might, however, require a further study to test against the sensitivity of these electron capture rates. The present set of Fuller, Fowler \& Newman (1982) is based on estimates for average properties of the Gamow-Teller giant resonance rather than on more secure shell model calculations for fp-shell nuclei in the Fe-group (Dean et al. 1998).

One of the problems of nucleosynthesis calculations which follow a thermonuclear evolution on long timescales through high-temperature regimes is the lack of accuracy. This lack of accuracy is due to the fact that in 
such situations, where actually a nuclear statistical equilibrium should exist, the cancellation of huge opposing rate flows is only attained up to machine accuracy (i.e., $\approx 10^{-12}-10^{-13} \times$ the term size). In a similar way the term $1 / \Delta t$, appearing in the diagonal of the Jacobi matrix of the multidimensional Newton-Raphson iteration (Hix \& Thielemann 1999b), can become numerically negligible in comparison to reaction rate terms in the same sum, which leads to numerically singular matrices. For that reason, we have adopted here the accurate solution, i.e., we followed the nuclear evolution with a screened NSE network containing 299 species during periods when temperatures beyond $T=6 \times 10^{9} \mathrm{~K}$ are attained (see Hix \& Thielemann 1996, 1999a). This takes into account the changes in binding energy or reaction Q-values due to screening. Weak reaction rates (electron captures and beta-decays), which are not in an equilibrium and occur on longer timescales, were included by using the NSE abundances. In this way we could track accurately the evolution of the electron fraction $Y_{e}$.

\section{EXPLOSIVE NUCLEOSYNTHESIS}

\subsection{Slow Deflagration}

In the present study we follow the approach discussed above by varying the ignition density and the initial deflagration velocity and test specifically the effect on the Fegroup composition in the central part. Figure 4 shows the maximum densities and temperatures for the inner mass zones experiencing complete and incomplete $\mathrm{Si}$ burning in the models WS15, WS30, CS15, and W7 as a comparison. It can be recognized that WS15 and WS30 experience densities similar to that of $\mathrm{W} 7$, which is due to the common ignition densities of initial model W, with WS15 attaining the highest temperatures. CS15, based on initial model C, is shifted to smaller densities.

During the burning the central region undergoes electron captures on free protons and iron peak nuclei. The central densities, similar to W7 for both WS models but combined with higher temperatures, result in more energetic Fermi distributions of electrons and larger abundances of free protons. This leads to larger amounts of electron captures on free protons and nuclei and smaller central $Y_{e}$ values. Figure 5 shows the final $Y_{e}$ during charged-particle freeze-out and before long-term decay of unstable burning products. Table 2 lists this value of $Y_{\mathrm{e}}$ in the center, $Y_{\mathrm{e}, \mathrm{c}}$, together with nucleosynthesis information, which will be discussed later. In summary, $Y_{\mathrm{e}, \mathrm{c}}$ is lower for higher central densities and slower deflagrations, the latter leading also to higher central temperatures. Figure 5 and Table 2 also list two models WSL and WLAM that experience (partially or entirely) deflagration velocities as small as the laminar speed, which apparently do not follow this logic anymore. All models are discussed in detail in the following paragraphs.

WS15, corresponding to a slow deflagration with a burning-front propagation of $1.5 \%$ of the sound speed, reaches higher central temperatures than WS30. The case WS30, corresponding to a burning-front propagation of $3 \%$ of the sound speed, has similar densities, but slightly lower temperatures for each radial mass zone in comparison to WS15 (also for a shorter duration). Besides the different central value, both models also have a different central $Y_{e}$ gradient (see Fig. 5). The WS15 curve, with lower central values, reaches $Y_{e}=0.4985$ (inherited from the progenitor white dwarf after He burning) at smaller radii than WS30. A smaller deflagration speed causes a later arrival of the burning front at a given mass coordinate. Thus, matter at this mass coordinate has a longer time to preexpand between the arrival of the central information with sound speed and the arrival of the burning front. Therefore, burning occurs there at a lower density (and temperature) with smaller average electron energies, causing less electron capture. This means that a smaller propagation speed produces a smaller central $Y_{e}$, but reaches $Y_{e}=0.4985$ also at smaller radii, i.e., produces a steeper $Y_{e}$ gradient. W7, with a different description of the burning-front velocity, but on average a larger speed, led to a higher central $Y_{e}$ and a flatter $Y_{e}$ gradient.

CS15 and CS30, which also corresponds to a burning front propagation with $1.5 \%$ or $3 \%$ of sound speed, behave in a fashion very similar to WS15 and WS30 but are characterized by a smaller central ignition density. Because of a smaller ignition density the central $Y_{e}$ values are larger. Otherwise the $Y_{e}$-gradients are the same for CS15 and WS15 as well as CS30 and WS30, each pair having the same burning-front speed. CS50, a case with $5 \%$ of sound speed, has a more extended region of decreased $Y_{e}$ out to larger masses (coming close to the behavior of W7), but because of the lower ignition density and lower central temperatures the central $Y_{\mathrm{e}, \mathrm{c}}$ is larger again.

WSL is an additional case and corresponds to a calculation with an initially laminar flame speed (out to $0.05 \mathrm{M}_{\odot}$, see Fig. 5), before an artificial acceleration is induced via turbulent mixing of ashes. WSLAM starts in exactly the same manner; however, the front stays laminar, i.e., only the minimum flame speed is permitted. According to the previous discussion one would expect an even steeper $Y_{e}$-gradient, and thus a lower central $Y_{e}$, owing to higher temperatures during a longer duration time. The steeper gradient can be seen outside $0.06 \mathrm{M}_{\odot}$ for WSL, where the front starts to accelerate beyond the laminar speed. Inside the range of $0.06 \mathrm{M}_{\odot}$ the $Y_{e}$ is almost constant and larger than e.g., in WS15, which experienced the same ignition density and larger flame speeds. There are two reasons for this behavior in the very central zones:

1. Initially during the burning, the lowest central $Y_{e^{-}}$ values of $\sim 0.432$ are obtained as expected, but this value is - in contrast to all other cases encountered previously - smaller (more neutron-rich) than the Fe-group nuclei in the valley of stability (see the most neutron-rich entries in Table 2, i.e., ${ }^{50} \mathrm{Ti},{ }^{54} \mathrm{Cr},{ }^{58} \mathrm{Fe}$, and ${ }^{64} \mathrm{Ni}$ with $Z / A$ values of $0.44,0.444,0.448$, and 0.438 ). This leads to counterbalancing $\beta^{-}$-decays which win against electron captures as densities and temperatures (slowly) decrease while matter is still in an NSE. This causes an increase in $Y_{e}$ to the displayed values before charged-particle freeze-out (see Fig. 6).

2. During the laminar front propagation, the large amount of central electron captures leads to neutrino losses which reduce the local energy release to a point where the expansion is very small (see the time dependence of $\rho$ and $T$ in Fig. 1). This keeps temperatures and densities high for a prolonged period, and matter stays in an NSE (the differences between WSL and WLAM emerge when the 
burning front is accelerated in WSL beyond the laminar speed). This NSE distribution of nuclei - with a total $Y_{e}$ more neutron-rich than stable Fe-group nuclei - permits $\beta^{-}$-decay of short-lived nuclei toward a total $Y_{e}$ corresponding to neutron-rich, stable Fe-group muclei (beyond 0.44 ) as long as temperatures are high enough to ensure an NSE. This results in the surprising fact that a burning front that stays laminar (WLAM) causes a higher $Y_{e}$ after charged-particle freeze-out than WSL (see changes in Fig. 6 after $20 \mathrm{~s}$ ). Thus, a very small $Y_{e}$, which produces e.g., large amounts of ${ }^{48} \mathrm{Ca}(Z / A=0.417)$, can only be attained in models with a higher ignition density, causing large amounts of electron capture, but with nonlaminar burning fronts that permit a fast expansion rather than keeping matter in NSE for a long time.

With the exception of the laminar burning-front models just discussed, which show a different behavior in Figure 5, we can summarize the major options for slow deflagrations to change $Y_{e}$ values in the central part of SNe Ia explosions: (1) the burning-front speed determines the $Y_{e}$ gradient, and the slowest speeds lead to the smallest central values; (2) lower central ignition densities cause larger $Y_{e}$ values, with the gradient, however, depending only on the propagation speed. These features hold true as long as $Y_{e}$ values in explosive burning do not drop below 0.44 , when competing $\beta^{-}$-decays have also to be taken into account. While the correct conditions occurring in SNe Ia might be still forthcoming from detailed multidimensional hydrodynamic calculations, this parameter study shows how nucleosynthesis results can give important clues to $v_{\text {def }}$ and $\rho_{\text {cign }}$.

Figures 8ab, 9ab, 10ab, and 11ab show abundance plots (mostly of Fe-group nuclei) for the central parts of CS15, WS15, CS30, WS30, CS50, W7, WSL, and WLAM where electron capture plays an important role. We see that $Y_{e}$ values of 0.47-0.485 lead to dominant abundances of ${ }^{54} \mathrm{Fe}$ and ${ }^{58} \mathrm{Ni}$; values between 0.46 and 0.47 produce dominantly ${ }^{56} \mathrm{Fe}$; values in the range of 0.45 and below are responsible for ${ }^{58} \mathrm{Fe},{ }^{54} \mathrm{Cr},{ }^{50} \mathrm{Ti}$, and ${ }^{62,64} \mathrm{Ni}$; and values below $0.43-0.42$ are responsible for ${ }^{48} \mathrm{Ca}$. Figure 5 clearly indicates that because of the flat $Y_{e}$-gradient of W7, the total amount of matter experiencing the range of $Y_{e}=0.47$ 0.485 is much larger than in cases with slower deflagration speeds and larger $Y_{e}$-gradients. WS15 and CS15 have similar total amounts of ${ }^{54} \mathrm{Fe}$ and ${ }^{58} \mathrm{Ni}$, but at slightly different locations (see the $Y_{e}$-range in Fig. 5 and the different mass scales in Figs. 8a and 8b). WS30 and CS30 contain a larger amount of ${ }^{54} \mathrm{Fe}$ and ${ }^{58} \mathrm{Ni}$, owing to the flatter

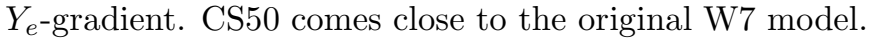

\subsection{Fe-Group Composition in Slow Deflagrations}

One of the motivations for the present exercise is to get constraints from comparison with solar Fe-group abundances. This can provide a better understanding of the burning-front propagation and test how the otherwise quite compelling features of the widely used model W7 can be improved. Figure 12 shows the ratio of abundances produced in W7 to solar abundances. These are the results of recalculations of the original W7 with the present reaction rate library and an increased accuracy in mass conservation in comparison to earlier studies due to the screened NSE treatment at high temperatures discussed in $§ 2.4$. Displayed are abundance ratios after the decay of unstable nuclei, normalized to unity for ${ }^{56} \mathrm{Fe}$. If SN Ia events are a relatively homogeneous class, the comparison of nucleosynthesis products with solar abundances is actually meaningful without averaging over a complete sample.

It is immediately obvious from Figure 12 that the production of Fe-group nuclei in comparison to their solar values is a factor of 2-3 larger than the production of intermediate nuclei from $\mathrm{Si}$ to $\mathrm{Ca}$. When considering that $\mathrm{SNe}$ Ia produce about $0.8 \mathrm{M}_{\odot}$ of Fe-group nuclei in comparison to $\approx 0.1 \mathrm{M}_{\odot}$ from SNe II, and that the Ia/(II+Ibc) ratio is about 0.15-0.27 in our galaxy (van den Bergh \& Tammann 1991; Cappelaro et al. 1997), SNe Ia are responsible for more than $55 \%$ of Fe-group nuclei. Thus, even if an isotope has no contribution from SNe II, this implies that the isotopic ratios among the Fe-group in the SNe Ia ejecta should not exceed the solar ratios by a factor of $\sim 2$, in order to result in solar ratios for $\mathrm{SNe}$ II $+\mathrm{SNe}$ Ia. If we assume on the other hand that an (Fe-group) isotope is made by SNe II in solar proportions, then it has also to be made in solar proportions in SNe Ia to obtain the solar mix for the sum of both contributions. An overproduction by SNe II would even ask for an underproduction in $\mathrm{SNe}$ Ia. If we are conservative and neglect the latter case, an overproduction of a factor 1-2 in SNe Ia would be permitted. Multiplying this with an average uncertainty factor of 2 would permit overproductions of $2-4$, dependent on the fact whether an SNe II contribution is existing or not. In general we do not know this at present, and we take an overproduction factor in SNe Ia of $\sim 3$ as an alarm sign for nucleosynthesis constraints. In this respect, we notice in Figure 12 the overproduction of ${ }^{54} \mathrm{Cr}$ and ${ }^{58} \mathrm{Ni}$ by a factor of $\sim 4-5$. Here ${ }^{54} \mathrm{Cr}$ is an $N=Z+6$ nucleus originating from the very central regions with low $Y_{e}$, while ${ }^{58} \mathrm{Ni}$ (and ${ }^{54} \mathrm{Fe}$ ) are nuclei with $N=Z+2$ measuring the bulk neutron excess of the material affected by the deflagration wave in the intermediate $Y_{e}$ range $\sim 0.48$ (see also Table 2 ). Outside of $0.3 \mathrm{M}_{\odot}$ in the exploding white dwarf, where electron capture is not effective, the neutron excess is only determined by the ${ }^{22} \mathrm{Ne}(N=Z+2)$ admixture to ${ }^{12} \mathrm{C}$ and ${ }^{16} \mathrm{O}$ in the original composition, stemming from ${ }^{14} \mathrm{~N}$ in $\mathrm{He}$ burning, which in turn originated from all $\mathrm{CNO}$-nuclei in $\mathrm{H}$ burning. The $Y_{e}$ or neutron-excess $\eta$ outside $0.3 \mathrm{M}_{\odot}$ is thus a measure of the metal abundance (nuclei heavier than $\mathrm{He}$ ) and the galactic age of the white dwarf. The quoted calculations were performed with $X\left({ }^{22} \mathrm{Ne}\right)=0.025$, which corresponds to $\eta$ of $\sim 30 \%$ higher than the solar metallicity, thus overestimating the average value for "old" white dwarfs undergoing a SN Ia event.

Using an averaged metallicity of SN Ia progenitor systems less than solar would reduce the overproduction of these nuclei. This is shown in Figures 13 for a deflagration model like W7 but with zero metallicity (W70). Figures $14 \mathrm{a}$ and $14 \mathrm{~b}$ give the comparison of the abundance distributions. Bravo et al. (1992) have performed a similar test. As the total SNe Ia contribution in our galaxy is given by an integral over time or metallicity up to the formation of the solar system, one expects average metallicities of 0.5-0.6 times solar. This brings the ${ }^{54} \mathrm{Fe} /{ }^{56} \mathrm{Fe}$, ${ }^{57} \mathrm{Fe} /{ }^{56} \mathrm{Fe},{ }^{58} \mathrm{Ni} /{ }^{56} \mathrm{Fe}$, and ${ }^{62} \mathrm{Ni} /{ }^{56} \mathrm{Fe}$ ratios within a factor of 2-3 of solar, respectively, which corresponds to the present uncertainty range of thermonuclear reaction rates 
and the minimum $\approx 50 \%$ contribution of $\mathrm{SNe}$ Ia to the $\mathrm{Fe}$ group. It does not reduce the ${ }^{54} \mathrm{Cr}$ abundance sufficiently, which originates solely from the central layers, where $Y_{e}$ is the smallest and entirely due to electron captures rather than the metallicity in terms of ${ }^{22} \mathrm{Ne}$. An interesting aspect of the change in metallicity, leading to reductions of ${ }^{54} \mathrm{Fe}$ and ${ }^{58} \mathrm{Ni}$, is the varying amount of early $\mathrm{Fe}\left({ }^{54} \mathrm{Fe}\right.$ before ${ }^{56} \mathrm{Ni}$ decay) and late $\mathrm{Ni}\left({ }^{58} \mathrm{Ni}\right.$ after ${ }^{56} \mathrm{Ni}$ decay) in SN Ia ejecta, leading to features that can be analyzed in observed spectra (see Höflich et al. 1998). This analysis covers one uncertainty regarding the initial composition. Another one would be a variation of the initial ${ }^{12} \mathrm{C} /{ }^{16} \mathrm{O}$ ratio, depending strongly on the initial white dwarf mass and metallicity. This has also been addressed recently by Umeda et al. (1999) and Höflich et al. (1999). Thus, while the metallicity and initial composition are one set of parameters, the ignition density and burning-front velocity represent another set, as outlined in $\S 2$. A burning front with a smaller velocity could reduce the amount of material in the $Y_{e}$ range $0.47-0.485$, where ${ }^{54} \mathrm{Fe},{ }^{58} \mathrm{Ni}$, and ${ }^{62} \mathrm{Ni}$ are produced in large amounts (see e.g., Table 2).

In Figures 15a and 15b, 16a and 16b, 17, and 18a and $18 \mathrm{~b}$ the ratios to solar abundances (normalized to ${ }^{56} \mathrm{Fe}$ ) are displayed. Here the results of the central slow deflagration studies have been merged with (fast deflagration) W7 compositions for the outer layers, where $Y_{e}$ is given by the initial ${ }^{22} \mathrm{Ne}$ and not by electron captures. Thus, these are not yet full delayed detonation models (which will be discussed in the next subsection), but more preliminary approximations to test the central Fe-group results. In comparison to Figure 12 we see that in all cases the ${ }^{58} \mathrm{Ni}$ problem is strongly reduced and would be fully resolved when using also smaller metallicities (see W7 vs. W70). This is due to the steeper $Y_{e}$ gradient which produces less matter in the intermediate $Y_{e}$ range 0.47-0.485. The smaller propagation speed has, however, also the consequence that $Y_{e}$ dips deeper in the central layers of WS15 and WS30 than in W7. Such $Y_{e}$ 's lead to the overproduction of ${ }^{50} \mathrm{Ti}$ and ${ }^{54} \mathrm{Cr}$. This is not the case for CS15 and CS30, due to the lower ignition density.

Another interesting point surfaces, which was also addressed preliminarily in Thielemann et al. (1996) and also Meyer, Krishnan, \& Clayton (1996) and Woosley (1997b). If one takes the results of presently existing and still crude SNe II nucleosynthesis calculations from initiated explosions as well as the results of W7, it turns out that for some intermediate mass and all Fe-group elements the most neutron-rich nuclei are drastically underproduced. The central $Y_{e}$ values of slow deflagration models comes close to conditions, where these nuclei are produced in a normal freeze-out. This has the effect that nuclei like ${ }^{50} \mathrm{Ti}$, ${ }^{54} \mathrm{Cr},{ }^{58} \mathrm{Fe}$ and partially ${ }^{64} \mathrm{Ni}$ or ${ }^{48} \mathrm{Ca}$ are produced for $Y_{e}$ values below 0.46 ( or $\eta=1-2 Y_{e}=0.1$ ). Whether this leads after integration over all mass zones just to solar abundances or to a strong overproduction will be tested here (see also Table 2). Khokhlov et al. (1992) undertook already a preliminary assessment of this question but did not consider all of these nuclei in their calculations. Woosley (1997b) tested models with higher ignition densities and burning-front velocities, whether such elements can be produced. Here we take again the philosophy to obtain constraints for the "average" case of SNe Ia, to test on the one hand whether they can produce such isotopes at all, and if so, whether constraints can be set for the conditions in order to avoid overproductions beyond the often mentioned factor of 3 .

It is a well-known fact that SNe Ia are not identical (see e.g., Hamuy et al. 1995) and that therefore a continuous superposition of such models has to be responsible for the solar Fe-group composition. On the other hand, we also know that the majority of SNe Ia come from a narrow window of conditions (Branch 1998), i.e., the notion of an average SN Ia event makes sense. If Fe-group elements are produced by SNe Ia to $\sim 55 \%$ and Ia's were the only sources of the neutron-rich Fe-group nuclei, we must conclude that an overproduction of a factor of 3 is permitted and that a larger overproduction has to be avoided for the average event. We see that for the cases discussed before (CS15, WS15, CS30, WS30) ${ }^{54} \mathrm{Fe}$ and ${ }^{58} \mathrm{Ni}$ can be produced within the permitted uncertainty limits $\left({ }^{58} \mathrm{Ni}\right.$ however having a tendency for overpoduction). These are the nuclei with intermediate $Y_{e}(0.47-0.485)$, which measure the $Y_{e}$ gradient via the amount of mass contained in that interval. CS50, which has a larger burning-front speed and a flatter slope, starts to overproduce these nuclei.

The more neutron-rich Fe-group nuclei depend more on the central layers, which experience lower $Y_{e}$-values. ${ }^{54} \mathrm{Cr}$ is produced within permitted limits in CS15 and CS30 but is overproduced in WS15 and WS30 owing to the higher ignition densities and central $Y_{e}$ values that are too low. ${ }^{50} \mathrm{Ti}$ is produced close to solar values for CS15, underproduced by CS30, well produced for WS30, and clearly overproduced in WS15, preferring the central $Y_{e}$ values of CS15 and WS30, which are similar. ${ }^{64} \mathrm{Ni}$ is essentially only made in large quantities for such low- $Y_{e}$ conditions as in WS15. Close to solar values cannot be attained for ${ }^{48} \mathrm{Ca}$ in any of these models, and its production would require lower $Y_{e}$ values. An attempt to do this was a purely laminar, i.e., the slowest possible, burning front. The display in Figure 18 a and 18b makes clear that this is not possible, as discussed in $\S 3.1$. The main reason is that such conditions produce nuclei which are unstable against $\beta^{-}$-decay. In intermediate phases sufficiently low $Y_{e}$ values are attained as a result of electron captures. NSE or Quasi-statistical equilibrium (QSE) redistributes abundances according to the then obtained smaller $Y_{e}$ values. $\beta^{-}$-decay of the short-lived neutron-rich isotopes leads to an increase in $Y_{e}$ during the expansion, when the densities and temperatures (and therefore the electron capture rates) decrease, before freeze-out from charged particle reactions and NSE/QSE (see Fig. 6). Thus, to produce a nucleus like ${ }^{48} \mathrm{Ca}$ in sufficient amounts, only very highdensity ignitions (followed by a fast expansion to avoid the influence of $\beta^{-}$-decays) of progenitor systems, which barely avoid an accretion induced collapse (AIC), might be responsible (see, e.g., Nomoto \& Kondo 1991; Woosley 1997b).

From this exercise we see that not all of these neutronrich nuclei can be made in similar proportions for one set of deflagration parameters unless a detailed fine-tuning of $v_{\text {def }}(r)$ and $Y_{e}(r)$ is performed or multidimensional propagation of the burning front produces exactly a superposition of conditions as needed to fit all abundance constraints. In general CS15 and CS30 seem to be better 
models than WS15 and WS30 in terms of avoiding a large overproduction of neutron-rich elements. The burningfront speed in the central layers seems to be constrained to values below $5 \%$ of the sound speed in order to avoid the overproduction of ${ }^{58} \mathrm{Ni}$ (see CS50 and Figure 17 as well as Table 2 with comparable problems found for W7 and W70). If on the other hand there were no other sites than SNe Ia to produce isotopes like ${ }^{50} \mathrm{Ti},{ }^{54} \mathrm{Cr},{ }^{58} \mathrm{Fe},{ }^{64} \mathrm{Ni}$ or ${ }^{48} \mathrm{Ca}$, we would have to overproduce in comparison to solar by about a factor of 2 , and some features of the models WS15, WS30 and even higher density events were needed, which can fill in the remaining deficiencies. Thus, one would need the majority of events similar to CS15 or CS30 and a smaller number of higher density ignitions to produce the more neutron-rich nuclei. This could guarantee on the one hand some overproduction, which (in combination with SNe II) would permit solar abundances in total, and avoid on the other hand not-permitted overproduction.

\subsection{Delayed Detonation}

The final aim, after constraining the central slow deflagration part of $\mathrm{SNe}$ Ia, is to find also composition constraints for the deflagration detonation transition (DDT). As discussed in $\S 2.3$, we chose transition densities of 3.0, 2.2 , and $1.7 \times 10^{7} \mathrm{~g} \mathrm{~cm}^{-3}$ for the given models WS15 and CS15, which turn them into WS15DD3, WS15DD2, WS15DD1 or CS15DD2 and CS15DD1. Figures 19 - 21 show the abundance distributions of slow deflagrations combined with delayed detonation models against the expansion velocity and $M_{r}$ of DD models. The central regions of these models have been shown before in Figures 8 and 9 , thus the abundance distributions of neutron-rich species such as ${ }^{54} \mathrm{Cr},{ }^{50} \mathrm{Ti},{ }^{58} \mathrm{Fe}$, and ${ }^{62} \mathrm{Ni}$ are not repeated here.

As the deflagration wave propagates outward, the white dwarf gradually expands to undergo less electron capture and thus mostly ${ }^{56} \mathrm{Ni}$ is synthesized. Eventually, the deflagration enters the region of incomplete Si burning and explosive O-Ne-C-burning, where the transition to a detonation occurs. For comparison the abundance distributions of W7 and W70 were shown in Fugure 14. The total masses of ${ }^{56} \mathrm{Ni}\left({ }^{56} \mathrm{Fe}\right)$ produced in these combined models have been summarized in Table 1 .

These theoretical abundance distributions can be compared with the observed expansion velocities of several elements as estimated from supernova spectra. It is seen that WDD2 and WDD1 produce two Si-S-Ar peaks at low velocity $\left(\sim 4,000 \mathrm{~km} \mathrm{~s}^{-1}\right)$ and high velocities (10,000 $\left.15,000 \mathrm{~km} \mathrm{~s}^{-1}\right)$. The intermediate mass elements at low velocities are important to observe at late times in order to distinguish between models. In particular, the minimum velocity of Ca in WDD models is $\sim 4,000 \mathrm{~km} \mathrm{~s}^{-1}$, which would be higher for a faster deflagration.

The Ca velocities should be compared with the observed minimum velocities of $\mathrm{Ca}$ indicated by the red edge of the Ca II H and K absorption blend (Fisher et al. 1995). The lowest velocities of $\mathrm{O}$ and $\mathrm{Mg}$ also provide interesting constraints. For example, SNe 1990N, 1992A, and 1991T show $\mathrm{O}$ in the wide velocity range from $\approx 10,000$ to $20,000 \mathrm{~km}$ $\mathrm{s}^{-1}$ (Leibundgut et al. 1991a; Jeffery et al. 1992; Mazzali et al. 1993; Kirshner et al. 1993). For W7 and WDDs, the minimum $\mathrm{O}$ velocity is $12,000-15,000 \mathrm{~km} \mathrm{~s}^{-1}$. The observed $\mathrm{O}$ velocity as low as $10,000 \mathrm{~km} \mathrm{~s}^{-1}$ may indicate a mixing of $\mathrm{O}$ in the velocity space.

Meikle et al. (1996) have observed a P Cyg-like feature at $\sim 1.05 / 1.08 \mu \mathrm{m}$ in SN $1994 \mathrm{D}$ and 1991T. They note that, if this feature is due to He, He in SN 1994D is likely to be formed in an alpha-rich freeze-out and mixed out to the high-velocity layers $\left(\sim 12,000 \mathrm{~km} \mathrm{~s}^{-1}\right)$. The maximum velocity of $\mathrm{He}$ is $5,000-6,000 \mathrm{~km} \mathrm{~s}^{-1}$ in WDDs, being slower than $\sim 9,000 \mathrm{~km} \mathrm{~s}^{-1}$ in W7, so that more extensive mixing of He would be required for WDDs than in W7. (Note that ${ }^{4} \mathrm{He}$ is plotted neither in Fig. 14 nor in Figs. 19 - 21, but its location can be easily identified with the region where the ${ }^{58} \mathrm{Ni}$ abundance forms a plateau up to the point where it turns over to ${ }^{54} \mathrm{Fe}$ on the same level. This is the transition from alpha-rich freeze-out to incomplete Si burning.) Alternatively, if the feature is due to $\mathrm{Mg}$, the $\mathrm{Mg}$ velocity is confined to $12,500-16,000 \mathrm{~km}$ $\mathrm{s}^{-1}$ in SN 1994D, which is consistent with W7 (13,000 $15,000 \mathrm{~km} \mathrm{~s}^{-1}$ ). For WDDs, on the other hand, the minimum velocities of $\mathrm{Mg}$ are 14,500 $\mathrm{km} \mathrm{s}^{-1}$ (WDD1), 16,500 $\mathrm{km} \mathrm{s}^{-1}$ (WDD2), and 18,000 $\mathrm{km} \mathrm{s}^{-1}$ (WDD3), and the latter two models seem to have too high velocities.

Mixing of ejected material in velocity space could occur convectively during the propagation of the deflagration wave (Livne 1993). Nonspherical explosions induced by delayed detonations could also produce nonspherical abundance distribution, i.e., elemental mixing in the velocity space. From the calculated synthetic spectra and their comparison with the observations, more advanced methods (Harkness 1991) do not favor mixing opposite to initial suggestion by Branch et al. (1985).

\section{YIELDS OF SNE IA AND GALACTIC CHEMICAL EVOLUTION}

\subsection{Features of SN Ia Nucleosynthesis}

Complete isotopic compositions of WDD and CDD models are given in Table 3. Table 3 assumes full decay of all unstable species. We provide separately in Table 4 abundances of long-lived radioactive nuclei, of importance either for gamma-ray detection, extinct radioactivities, or chemical evolution. The abundances are compared with solar abundances in Figures 22 - 24, which are normalized to ${ }^{56} \mathrm{Fe}$. These Figures complement the earlier Figures 15 - 18, where the fast deflagration W7 was utilized in the outer layers rather than delayed detonation models. The major conclusions on the Fe-group composition remain the same as discussed before, in $\S 3.1$, owing to the fact that they are given by the central slow deflagrations. What can be studied here is the additional variation in the ratio of Fe-group to intermediate mass nuclei, which depends on the deflagration-detonation transition. Of course the variation in ${ }^{56} \mathrm{Fe}$ (originating from ${ }^{56} \mathrm{Ni}$ ) in the outer detonation layers also influences the ratio of neutron-rich Fegroup isotopes (from central locations) to ${ }^{56} \mathrm{Fe}$. Table 3 includes besides all DD-models (CDD1 short for CS15DD1 etc.) also W7 and W70 updated with the latest reaction rate set and improved accuracy by using a screened NSE treatment for long duration times at temperatures beyond $6 \times 10^{9} \mathrm{~K}$ (Hix \& Thielemann 1996). The ratios to solar abundances for W7 and W70 were shown in Figures 12 and 13. The essential features due to the DDT, as displayed 
in Figures 22 - 24 and in Table 3 can be summarized as follows:

1. The synthesized amount of $\mathrm{Fe}$ and thus the ratio between the intermediate mass elements and $\mathrm{Fe}, \mathrm{Si}-\mathrm{Ca} / \mathrm{Fe}$, is sensitive to the transition density from deflagration to detonation, as was already shown in Table 1. Among the WDD models, WS15DD2 produces only about $25 \%$ more ${ }^{56} \mathrm{Ni}$ than $\mathrm{W} 7\left(\sim 0.6 \mathrm{M}_{\odot}\right)$ but more Si-Ca than $\mathrm{W} 7$ by $40 \%$ (Fig. 23b), since more oxygen is burned in the outer layers. Therefore, the $\mathrm{Si}-\mathrm{Ca} / \mathrm{Fe}$ ratios are moved up to a certain extent. WS15DD1 has even larger $\mathrm{Si}-\mathrm{Ca} / \mathrm{Fe}$ ratios, which are close to solar ratios (Fig. 23a). This is not indicated for SNe Ia, owing to observations of low-metallicity stars reflecting the average SN II behavior (a reasoning outlined below in more detail). However, direct observations of the $\mathrm{Si}-\mathrm{Ca} / \mathrm{Fe}$ ratio in SNe Ia remnants (Tycho, SN 1006, etc.) are highly important and needed in order to distinguish between the models (Hughes et al. 1995; Miyata et al. 1998; Hwang, Hughes \& Petre 1998).

2. Neutron-rich species such as ${ }^{54} \mathrm{Cr}$ and ${ }^{50} \mathrm{Ti}$ are mostly produced in the slow deflagration phase. The degree of their overproduction with respect to ${ }^{56} \mathrm{Fe}$ depends also on the mass of ${ }^{56} \mathrm{Ni}$ produced in the outer detonation layers, as seen in Figure 24. This also explains why in Table 2 the entries for the same central models (WS15, CS15 etc.) change for neutron-rich species, although the central part from which these neutron-rich species originate is unaffected by the detonation. The reason is that the ratios in comparison to ${ }^{56} \mathrm{Fe}$ are taken.

3. There are some Fe-group contributions from alpharich freeze-out and incomplete Si-burning layers that depend on the DDT. Figures 19 - 21 show that the mass region experiencing incomplete $\mathrm{Si}$ burning (indicated by the ${ }^{54} \mathrm{Fe}$ plataeu) decreases in the sequence DD1-DD3. The region indicated by the ${ }^{58} \mathrm{Ni}$ plateau experiences alpharich freeze-out (the He abundance is not shown here) and increases in the sequence DD1-DD3. ${ }^{52} \mathrm{Fe}$ (decaying to the dominant $\mathrm{Cr}$ isotope ${ }^{52} \mathrm{Cr}$ ) and ${ }^{55} \mathrm{Co}$ (decaying to the only stable $\mathrm{Mn}$ isotope ${ }^{55} \mathrm{Mn}$ ) are typical features of incomplete Si burning. ${ }^{59} \mathrm{Cu}$ (decaying to the only stable Co isotope ${ }^{59} \mathrm{Co}$ ) is a typical feature of an alpha-rich freeze-out. For these reasons we see the strongest ${ }^{52} \mathrm{Cr}$ and ${ }^{55} \mathrm{Mn}$ overabundances in DD1 and the strongest appearance of ${ }^{59} \mathrm{Co}$ (while still underabundant) in DD3.

\subsection{The Role of SN Ia and SN II Contributions}

The chemical evolution of galaxies is dominated by its main contributors SNe II, SNe Ia, and planetary nebulae. The latter do not contribute to the element abundances in the range $\mathrm{O}$ through $\mathrm{Ni}$ (although a few specific minor isotopes can be produced in the s-process). Thus for the aspects considered here, we have to explain galactic evolution and also solar abundances by the combined action of SNe II and Ia. The ratio of Fe-group elements to Si-Ca in $\mathrm{SNe}$ Ia is of specific importance, in order to see how the overabundance of $\mathrm{O}-\mathrm{Ca} / \mathrm{Fe}$ (in comparison to solar) in SNe II can be compensated. Combined nucleosynthesis products of SNe Ia and SNe II with varying ratios can be compared to solar abundances. An important aspect for such an undertaking is, however, to test the individual components against existing observations first, before trying to attain a good solar mix (with possibly wrong pre- dictions for the individual SN I and SN II components).

Although we do not have a good quantitative measure from SN Ia observations for the Fe-group to Si-Ca ratio, the fast deflagration model W7 seems to give a good overall agreement via synthetic spectra calculations with observed Ia spectra (see Branch 1998 for a review). W7 has specific deficiencies in the global isotopic Fe-group composition from the inner layers, as discussed before, e.g., with respect to ${ }^{58} \mathrm{Ni}$ and ${ }^{54} \mathrm{Cr}$, but this does not affect the element ratios too strongly.

A similar or even worse situation is found for the quantitative analysis of SN II spectra. However, an independent tool exists that measures the integrated SN II yields: observed surface abundances of low-metallicity stars, which witness the abundances of the interstellar medium at their point of formation during early galactic evolution, when only SNe II contributed. For metallicities in the range $-2<[\mathrm{Fe} / \mathrm{H}]<-1$ we expect the integrated (mass averaged) properties of SNe II (see e.g., Nakamura et al. 1999). Here $[\mathrm{x} / \mathrm{y}]$ is defined as $\log _{10}\left[(\mathrm{x} / \mathrm{y}) /(\mathrm{x} / \mathrm{y})_{\odot}\right]$. Such abundance features were reproduced with nucleosynthesis products of SNe II as a function of stellar mass, taken from the calculations by Nomoto \& Hashimoto (1988), Hashimoto et al. (1996), and Thielemann et al. (1996) as summarized in Hashimoto (1995), Tsujimoto et al. (1995), and Nomoto et al. (1997a). SNe II yields, integrated from $m_{l}=10 \mathrm{M}_{\odot}$ to $m_{u}=50 \mathrm{M}_{\odot}$ with a Salpeter IMF, are also given in Table 3. The upper mass bound $m_{u}$ is chosen to reproduce $[\mathrm{O} / \mathrm{Fe}]=+0.4$, which is consistent with the observations of low metallicity stars for $[\mathrm{Fe} / \mathrm{H}]<$ - 1. Such observations in low-metallicity stars give also the best constraints on average Fe-group abundances of SNe II, which are poorly known theoretically owing to the still existing lack of self-consistent core collapse supernova models. The representation of the Fe-group (beyond Ti) closest to the low metallicity observations seems to be the one of our $20 \mathrm{M}_{\odot}$ star (see Figure $5 \mathrm{~b}$ in Thielemann et al. 1996 and note that the observed Co abundance has come down to about -0.1, i.e., it shows a better agreement with the dashed line).

A possible deviation from solar ratios has to be made up by an opposite behavior of SNe Ia setting in at about $[\mathrm{Fe} / \mathrm{H}]=-1$ in order to attain solar values at $[\mathrm{Fe} / \mathrm{H}]=0$. Fe-group elements for which information is available are Ti, Sc, Cr, Mn, Co, Ni (Magain 1987, 1989; Gratton \& Sneden 1988, 1991; Gratton 1989; Zhao \& Magain 1990; Nissen et al. 1994). They lead to typical uncertainties of 0.1 dex and one finds average SN II values of $0.25,0$, $-0.1,-0.3,-0.1,-0.1$. Taken the typical uncertainty of 0.1 dex, this leaves $\mathrm{Ti}$ and $\mathrm{Mn}$ as elements with clear signatures for a SN II behavior different from solar, which asks for the opposite SN Ia behavior. Cu and Zn start having strong s-process contributions. We avoid their discussion because of these complications and because they are not a clear indication for the required SN Ia signature.

$\mathrm{Ti}$ is dominated by the isotope ${ }^{48} \mathrm{Ti}$ (see Table 3), i.e., we have to relate the element ratio $\mathrm{Ti} / \mathrm{Fe}$ to ${ }^{48} \mathrm{Ti} /{ }^{56} \mathrm{Fe}$. We see that for all models that underproduce $\mathrm{Si}-\mathrm{Ca}$ (as needed for SNe Ia), i.e., the DD2, DD3 and W7 models, ${ }^{48} \mathrm{Ti}$ is also underproduced by similar amounts. This agrees with the observational trend that $\mathrm{Ti}$ is dominantly produced by SNe II and can be understood from the ${ }^{48} \mathrm{Cr}$ abundances 
(decaying to the main $\mathrm{Ti}$ isoptope ${ }^{48} \mathrm{Ti}$ ). ${ }^{48} \mathrm{Ti}$ is only produced in a strong alpha-rich freeze-out as it occurs in SNe II. The small region of a weak alpha-rich freeze-out, indicated in Figure 4 , is not sufficient.

The tendency is not so clear for Mn. The only stable Mn isotope is ${ }^{55} \mathrm{Mn}$, typically produced from unstable ${ }^{55} \mathrm{Co}$. This isotope results (1) from incomplete Si burning with a relatively high $Y_{e}$ (e.g., 0.4985; see its production in SNe II in Fig. 1 in Thielemann et al. 1996 and the present Figs 19 - 21 in the incomplete Si-burning regions) or (2 from a somewhat reduced $Y_{e}$ (around 0.49) in complete $\mathrm{Si}$ burning (see Figs. 8-9). This can be understood within the framework of quasi-equilibrium groups in Si burning, in our case the $\mathrm{Si}$ and the Fe group. Incomplete burning leads to a small total abundance in the Fe group in comparison to the Si group. Hix \& Thielemann (1996) found that in such a case the composition in the Fe group is typically more neutron-rich than expected from the global $Y_{e}$. Hence, ${ }^{55}$ Co with a $Z / A=0.49$ is in incomplete burning also produced for conditions with a global $Y_{e}$ of 0.4985 . Both locations (in complete and incomplete burning) can be nicely seen in Figure 14, with the additional $Y_{e}$ information taken from Figure 5. As discussed before, the same is found in the central parts in Figures 8-9 and globally in Figures 19-21. The problem is, however, that with the exception of the DD1 models and W7, all DD models underproduce $\mathrm{Mn} / \mathrm{Fe}$ in comparison to solar, opposite to what is required from average SN II yields inferred from low-metallicity observations. It appears that the dominant source for $\mathrm{Mn}$ is the incomplete Si-burning region, which is most extended in the DD1 models.

${ }^{52} \mathrm{Cr}$ is the dominant nucleus of the $\mathrm{Cr}$ isotopes. DD2 shows a slight overabundance, DD1 a stronger overabundance. ${ }^{52} \mathrm{Cr}$ originating from ${ }^{52} \mathrm{Fe}$ is also a nucleus dominated by incomplete Si burning and therefore this behavior is understandable. The light underabundance in SNe II can thus be compensated by SNe Ia. Co from SNe II is slightly underproduced. All our calculations show an underabundance in SN Ia models. It seems that the alpharich freeze-out nucleus ${ }^{59} \mathrm{Cu}$ (decaying to the only stable Co isotope ${ }^{59} \mathrm{Co}$ ) is never produced sufficiently. However, there exists the same problem in SNe II (Nakamura et al. 1999) and possibly other (nuclear?) sources might be the origin of this behavior. ${ }^{58} \mathrm{Ni}$ originates from neutronrich central regions and partially from alpha-rich freezeout (where also ${ }^{62} \mathrm{Ni}$ is produced via ${ }^{62} \mathrm{Zn}$ decay). We have worked hard on our models to avoid an overproduction. Thus a slight underproduction in SNe II can easily be compensated. One should also have a look at Bravo et al. (1993) and Matteucci et al. (1993), who performed chemical evolution calculations and addressed some of these questions with the then existing observational and model constraints.

We finally want to return to neutron-rich Fe-group isotopes and point to a different witness of galactic evolution. While typically astronomical observations can give information only about element abundances (with a few exceptions from molecular lines in stars), there exists one source of isotopic information, the so-called isotopic anomalies in meteorites. They are usually contained in "inclusions" of primitive meteorites consisting of minerals with much higher melting temperatures than the surrounding mat- ter. This gives some indication that they originate from unprocessed "star dust" that survived temperatures in the early solar system and can give direct clues about its stellar origin. This is essentially proven for some $\mathrm{SiC}$ grains, graphites and diamonds (e.g., Zinner, Tang, \& Andrews 1989; Zinner 1995; Travaglio et al. 1999).

The nuclei ${ }^{48} \mathrm{Ca},{ }^{50} \mathrm{Ti},{ }^{54} \mathrm{Cr}$, and ${ }^{58} \mathrm{Fe}$, which play a crucial role in the central layers of SN Ia explosions, can also be found in isotopic anomalies (Lee 1988; Völkening \& Papanastassiou 1989, 1990; Loss \& Lugmair 1990). They occur, however, only in Ca-Al-rich inclusions whose history is less clear and some chemical processing in the early solar nebula has probably happened. Nevertheless, it is interesting to investigate whether these observed anomalies can lead to any indication of their origin. Harper et al. (1990) found a correlation with the r-process nucleus ${ }^{96} \mathrm{Zn}$. This would have pointed toward a SN II origin if the r-process originates from SNe II. On the other hand, Ireland (1994) found a probe where no correlation with r-process anomalies existed. Thus, no very clear indication for their SN II or SN Ia origin (or both?) presently exists. In the present paper, however, we applied the working hypothesis that their source is given by SN Ia ejecta, which is also indicated by the $Y_{e}$-constraints from SN II abundance observations (Thielemann et al. 1996). In case of an additional SN II contribution, even more stringent limits would exist.

\subsection{Ratios of SN Ia to SN II Events in the Galaxy}

We have full results for nucleosynthesis products of SNe Ia from the four models, WS15DD1-3, CS15DD1-2, W7, and W70, and the results are shown in Figures 22-24 and 12 - 13. CS30 and 50 as well as WS30 were calculated only for the central slow deflagration layers and not full delayed detonation models. This, as it affects the composition within the Fe group, might not be a sufficient quantitative basis with respect to relative abundances within the Fe group. But as the central deflagration speeds have minor influence on the total abundance of ${ }^{56} \mathrm{Ni}\left({ }^{56} \mathrm{Fe}\right)$, the different DD models are probably sufficient to determine the best $\mathrm{Si}-\mathrm{Ca} / \mathrm{Fe}$ ratios. The observational data by Gratton \& Sneden (1991) and Nissen et al. (1994) for $[\mathrm{x} / \mathrm{Fe}]$ at low metallicities $(-2<[\mathrm{Fe} / \mathrm{H}]-<1)$, x standing for elements from $\mathrm{O}$ through $\mathrm{Ca}$, show an enhancement of the alpha elements ( $\mathrm{O}$ through $\mathrm{Ca}$ ) by a factor of $2-3$ (0.3 to 0.5 dex in $[\mathrm{x} / \mathrm{Fe}])$ in comparison to Fe. This is the clear fingerprint of the exclusive contribution of SNe II in early galactic evolution. It has long been the aim of chemical evolution calculations to explain this behavior, among the most recent ones being Tsujimoto et al. (1995), Timmes, Woosley, \& Weaver (1995), Pagel \& Tautvaisine (1995, 1997), and Kobayashi et al. (1998).

Tsujimoto et al. (1995) tried to determine the ratio of SN Ia to SNe Ib+II events in the Galaxy by aiming for a best fit to an overal solar abundance pattern from $\mathrm{O}$ to $\mathrm{Ni}$. They made use of the mass-averaged SN II yields from 10 to $50 \mathrm{M}_{\odot}$ as shown in Table 3 and earlier results for $\mathrm{W} 7$. With the aid of a chemical evolution model they obtained a ratio $N_{\text {Ia }} / N_{\text {II }}=0.12$ of the total number of SNe Ia to SNe II (+Ib) that occurred in our Galaxy. This resulted from an overal best fit to the observed abundances and is consistent with the fact that the observed estimate of the SNe Ia frequency is as low as $10 \%$ of the total su- 
pernova rate. The observational constraints for this ratio range from values around 0.15 (van den Bergh \& Tammann 1991) to more recent determinations of 0.27 with errors of almost a factor of 2 (Cappelaro et al. 1997).

Rather than performing a full galactic evolution model, we want to concentrate on a typical abundance ratio, $\mathrm{Si} / \mathrm{Fe}$, and its contribution from SNe II and Ia. The averaged SNe II yields from our theoretical models summarized in Table 3 correspond to a $[\mathrm{Si} / \mathrm{Fe}]=0.347$. The results of the $20 \mathrm{M}_{\odot}$ model of Thielemann et al. (1996) would correspond to $[\mathrm{Si} / \mathrm{Fe}]=0.304$, which is close to the value of Gratton \& Sneden (1991) and Ryan, Norris, \& Beers (1996) of 0.3. One should, however, be aware of the typical uncertainties of 0.1 dex. Varying the SN Ia models among the above given list (W7, W70, DD1, DD2, and DD3) and making use of Si and Fe from the averaged SNe II ejecta or the $20 \mathrm{M}_{\odot}$ star, leads also to a required $\mathrm{Ia} / \mathrm{Ib}+\mathrm{II}$ ratio in order to obtain the solar mix of $\mathrm{Si} / \mathrm{Fe}$ with both contributions. This ratio is derived in the following way:

$$
\begin{aligned}
\left(\frac{M(\mathrm{Si})}{M(\mathrm{Fe})}\right)_{\odot} & =\frac{N_{\mathrm{Ia}} M_{\mathrm{Ia}}(\mathrm{Si})+N_{\mathrm{II}} M_{\mathrm{II}}(\mathrm{Si})}{N_{\mathrm{Ia}} M_{\mathrm{Ia}}(\mathrm{Fe})+N_{\mathrm{II}} M_{\mathrm{II}}(\mathrm{Fe})} \\
\frac{N_{\mathrm{Ia}}}{N_{\mathrm{II}}} & =\frac{M_{\mathrm{II}}(\mathrm{Si})-M(\mathrm{Si}) / M(\mathrm{Fe}) \odot M_{\mathrm{II}}(\mathrm{Fe})}{M(\mathrm{Si}) / M(\mathrm{Fe}) \odot M_{\mathrm{Ia}}(\mathrm{Fe})-M_{\mathrm{Ia}}(\mathrm{Si})}(4)
\end{aligned}
$$

The results for the obtained ratios are shown in Table 5. It is immediatey obvious that the DD1 models do not result in a physical solution. With their $\mathrm{Si}-\mathrm{Ca} / \mathrm{Fe}$ ratios being almost solar, they cannot compensate for the SNe II contribution, which have larger than solar values. There is a clear need for SNe Ia to produce ejecta with $\mathrm{Si}-\mathrm{Ca} / \mathrm{Fe}$ less than solar. Otherwise all models seem to be covered by the error bars for the $\mathrm{Ia} /(\mathrm{Ib}+\mathrm{II})$ ratios laid out by van den Bergh \& Tammann (1991) and Cappelaro et al. (1997). The DD3 values, especially for the $20 \mathrm{M}_{\odot} \mathrm{Si} / \mathrm{Fe}$ ratios being closest to the low-metallicity [Si/Fe], might somewhat indicate the lower limit. It should be noted that although the $\mathrm{Si} / \mathrm{Fe}$ ratios in the DD1 - DD3 models show a monotonous behavior (i.e., decline), this does not lead to a monotonous variation in $\mathrm{Ia} /(\mathrm{Ib}+\mathrm{II})$ ratios, owing to the form of equation (4), where differences and not the $\mathrm{Si} / \mathrm{Fe}$ ratios enter. This results in negative values for DD1 (due to a negative denominator) and goes through a maximum (when the denominator starts to be positive but is small) before decreasing to the DD3 values with an increasing denominator. This is due to the fact that Si masses decrease with the DD1 - DD3 sequence, while Fe increases.

With a low SN Ia frequency of $\mathrm{Ia} /(\mathrm{II}+\mathrm{Ib})=0.15$ and $\mathrm{W} 7$ abundances, ${ }^{56} \mathrm{Fe}$ from SNe Ia amounts to about $55 \%$ of total ${ }^{56} \mathrm{Fe}$ (see the discussion in §3.2). Larger values would increase this contribution. When assuming that SNe II do not produce neutron-rich species like ${ }^{54} \mathrm{Cr}$ and ${ }^{50} \mathrm{Ti}$ at all, we permitted for the abundance ratios between such nuclei and ${ }^{56} \mathrm{Fe}$ an overproduction factor of $\sim 4$. With the larger SN Ia frequencies of Table 5 and Cappelaro et al. (1997) such permitted overproductions would need to be further reduced and more stringent limits would apply.

\section{SUMMARY AND OUTLOOK}

From the very early days of explosive nucleosynthesis calculations, when no direct connection to astrophysical sites was possible yet, it was noticed (Trimble 1975) that the solar Fe-group composition could be reproduced with a superposition of matter from explosive Si burning with about $90 \%$ originating from a $Y_{e}=0.499$ source and $10 \%$ from a $Y_{e}=0.46$ source. We have discussed in some detail in the present paper that the central part of SNe Ia could be this second source, while Thielemann et al. (1996) have shown that SN II ejecta with $Y_{e}<0.498$ could cause serious problems.

New results by Hachisu et al. (1999a, 1999b; see also Li \& van den Heuvel 1997), which include wind losses in the interaction of binary systems, come to the conclusion that the majority of SNe Ia progenitor systems experience hydrogen accretion on white dwarfs at a rate that has them grow toward the Chandrasekhar mass through steady $\mathrm{H}-$ and He-burning. This leads to the single degenerate Chandrasekhar mass scenario (SD/Ch). Binary systems with steady H-burning on accreting white dwarfs and effective accretion rates as high as $\dot{M}>1 \times 10^{-7} \mathrm{M}_{\odot} \mathrm{yr}^{-1}$ might correspond to observed supersoft X-ray sources. This also would lead to low central ignition densities of the Chandrasekhar mass white dwarf at the thermonuclear runaway $\left(<2 \times 10^{9} \mathrm{~g} \mathrm{~cm}^{-3}\right)$, which correspond to the $\mathrm{C}$ series of the models discussed in the present paper. A small fraction can deviate from steady $\mathrm{H}$ burning and would experience weak hydrogen flashes near the end of the accretion history. Such cases would correspond to our W series of models and even higher ignition densities. Kobayashi et al. (1998) discussed metallicity effects and their influence on the delay time for the appearance of SNe Ia in galactic evolution.

Our nucleosynthesis results of the present paper are quite consistent with these scenarios and favor case $\mathrm{C}$. The reason is that too high ignition densities lead to a high degree of electron captures and small $Y_{e}$ values, which would cause an overproduction of ${ }^{54} \mathrm{Cr}$ and ${ }^{50} \mathrm{Ti}$ in excess of what is permitted for SNe Ia in a solar mix. We have to make two reservations here. New shell model calculations (Dean et al. 1998; Caurier et al. 1999) indicate that the electron capture rates could be substantially reduced in comparison to the rates by Fuller et al. $(1980,1982,1985)$ employed here. The application of these new capture rates might also permit models of our $\mathrm{W}$ series without serious deviations from the allowed central $Y_{e}$ values. In addition, such conclusions deal only with the dominant or average SN Ia events. If more neutron-rich nuclei like ${ }^{48} \mathrm{Ca}$ are also the result of SN Ia nucleosynthesis, an occasional event with a significantly higher ignition density is required, a fact also consistent with the above scenario.

Our nucleosynthesis results imply that for the dominant events the central density of the Chandrasekhar mass white dwarf at thermonuclear runaway must be as low as or lower than $\lesssim 2 \times 10^{9} \mathrm{~g} \mathrm{~cm}^{-3}$, though the exact constraint depends somewhat on the flame speed. Here is the point where nucleosynthesis predictions can also be of help for providing constraints to the supernova modeling and the burning-front velocity. A carbon deflagration wave, propagating as slow as $v_{\text {def }} / v_{\mathrm{s}} \sim 0.015$ or even slightly slower, would be the ideal choice for the neutronrich species such as ${ }^{54} \mathrm{Cr}$ and ${ }^{50} \mathrm{Ti}$; see cases CS15 and WS30. The latter case makes also clear that slightly larger ignition densities than $1.5 \times 10^{9} \mathrm{~g} \mathrm{~cm}^{-3}$ (case C) are per- 
mitted if the flame speed is increased appropriately. An acceleration of the flame speed in the outer part of the central layers is permitted to about $3 \%$ of the sound speed or slightly more, but should clearly stay below $5 \%$. For such fast burning fronts the $Y_{e}$-gradient becomes very flat and too much material in the range $Y_{e}=0.47-0.485$ is produced. This leads to dominant abundances of ${ }^{54} \mathrm{Fe}$ and ${ }^{58} \mathrm{Ni}$, a feature that was already prominent in $\mathrm{W} 7$, and causes an excessive overproduction of ${ }^{58} \mathrm{Ni}$ in galactic evolution. Our calculations were performed with a constant fraction of the sound speed. An acceleration of the burning front is expected (Khokhlov 1995; Niemeyer \& Woosley 1997) and future investigations should include such a time dependence, which is in agreement with the findings discussed above.

Finally, nucleosynthesis can also give clues about the deflagration-detonation transition (DDT). The most obvious consequence of choosing different transition densities is the amount of ${ }^{56} \mathrm{Ni}$ produced in a SN Ia event. Höflich and Khokhlov (1996) find from light curve modeling and spectra that the typical ${ }^{56} \mathrm{Ni}$ mass should be in the range $0.5-0.7 \mathrm{M}_{\odot}$. This agrees with W7. Among the DD models it would ask for a value somewhere between DD1 and DD2 (closer to DD2). Here 3, 2, and 1 stand for DDTs when densities ahead of the flame decrease to $3.0,2.2$, and 1.7 $\times 10^{7} \mathrm{~g} \mathrm{~cm}^{-3}$. DD1 is excluded for other reasons. The amount of $\mathrm{Si}-\mathrm{Ca}$ in comparison to $\mathrm{Fe}$ is too large in DD1 models in order to compensate for the well-known overproduction of Si-Ca in SNe II during galactic evolution. $\mathrm{Si} / \mathrm{Fe}$ ratios in $\mathrm{SN}$ Ia models require specific $\mathrm{Ia} /(\mathrm{II}+\mathrm{Ib})$ ratios in order to obtain a solar mix combined with SN II contributions (see Table 5). DD2 seems to be closest to the present observational constraints for this ratio by Cappelaro et al. (1997).

Small DDT densities favor larger amounts of matter that experience incomplete Si burning. Low-metallicity constraints require an overproduction of $\mathrm{Mn}$ (and $\mathrm{Cr}$ ) in $\mathrm{SNe}$ Ia. These elements are mostly made as ${ }^{55} \mathrm{Co}$ and ${ }^{52} \mathrm{Fe}$ (decaying to $\mathrm{Mn}$ and $\mathrm{Cr}$ ), which are favorably produced in incomplete Si burning and would also require a DDT between DD1 and DD2. (One should, however, realize that a fast deflagration could possibly simulate this as well see the Mn overproduction in Figure 12 - and on the other hand that these numbers would have to be rescaled or reinterpreted in multi-D calculations.) Thus, combining all requirements on the DDT from total $\mathrm{Ni}$ yields, $\mathrm{Si} / \mathrm{Fe}$ and $\mathrm{Ia} /(\mathrm{II}+\mathrm{Ib})$ ratios, as well as specific elements favored in incomplete Si-burning, we would argue for a DDT density slightly below $2.2 \times 10^{7} \mathrm{~g} \mathrm{~cm}^{-3}$, i.e., results between DD1 and DD2. One should, however, be careful with these constraints based on spherically symmetric approximations of the burning front. Full three-dimensional calculations could possibly produce the required ratio of matter from incomplete Si burning and complete Si burning with alpha-rich freeze-out in a different realization.

More extended calculations that make use of the con- clusions presented here, including a time dependence of $v_{\text {def }} / v_{\text {sound }}$, the best choice for the DDT density, and a detailed galactic evolution model, replacing our comparisons of only the global yields, will be the next step to undertake. That would also have to include further tests of the metallicity of the exploding object, a topic which has gained in importance with the cosmological interpretation of high-redshift SNe Ia. For this reason we repeat here in Figure 25a-25c some of the Figures 19-21 with a continuation to smaller mass fractions and purely plotted as a function of velocity, in order to magnify the behavior of the outer layers. The differences in velocities between CDD and WDD models are negligibly small in these plots.

As mentioned before, the ${ }^{54} \mathrm{Fe}$ (in the outer layers not affected by electron captures but only by the neutron excess due to the initial metallicity) ranging in velocities up to $15,000-19,000 \mathrm{~km} \mathrm{~s}^{-1}$ in the models DD1-DD3, is a strong indicator of metallicity (see Fig. 14). The minima in $\mathrm{S}, \mathrm{Ar}$, and $\mathrm{Ca}$, if observed in spectra, with positions between 16,000 and 21,000 $\mathrm{km} \mathrm{s}^{-1}$ in the DD1-DD3 models, could give further clues on the deflagration-detonation transition. And finally, any unburned intermediate mass elements at higher velocities would give a clear indication of the metallicity of the accreted matter. Our calculations and plots include only initial compositions of ${ }^{12} \mathrm{C},{ }^{16} \mathrm{O}$, and ${ }^{22} \mathrm{Ne}$. Solar abundances of Ca, Ar, S, Si, or Fe would correspond to mass fractions of $1.2 \times 10^{-4}, 1.6 \times 10^{-4}$, $7.6 \times 10^{-4}, 1.3 \times 10^{-3}$, or $2.3 \times 10^{-3}$.

Thus, any future investigations of very early time spectra, leading to abundance observations at high velocities, would provide strong constraints on the SNe Ia mechanism and the relation to the metallicity of the individual $\mathrm{SNe}$ Ia explosion.

\section{ACKNOWLEDGMENTS}

This work has been supported in part by the grantin-Aid for Scientific Research (05242102, 06233101) and COE research (07CE2002) of the Ministry of Education, Science and Culture in Japan, a fellowship of the Japan Society for the Promotion of Science for Japanese Junior Scientists (6728), the Swiss Nationalfonds (20-47252.96 and 2000-53798.98), the US National Science Foundation (grant PHY94-07194), and the DOE (contract DE-AC0596OR22464). Part of the computations were carried out on a Fujitsu VPP-500 at the Institute of Physical and Chemical Research (RIKEN) and the Institute of Space and Astronautical Science (ISAS), and a Fujitsu VPP-300 at the National Astronomical Observatory in Japan (NAO, Tokyo). We want to thank all participants of the NATO workshop on Thermonuclear Supernovae in Aiguablava, where the idea to the present research began. Some of us (K.I., K.N., W.R.H., and F.K.T.) thank the ITP at the University of California, Santa Barbara, for hospitality and inspiration during the supernova program. The paper was completed during the SNe Ia workshop at the Aspen Center for Physics (June 1999).

\section{REFERENCES}

Arnett, W.D. 1996, Nucleosynthesis and Supernovae (Princeton: Princeton Univ. Press)

Arnett, W.D., \& Livne, E. 1994a, ApJ, 427, 314

Arnett, W.D., \& Livne, E. 1994b, ApJ, 427, 330
Aufderheide, M., Fushiki, I., Woosley, S.E., Hartmann, D. 1994, ApJS, 91, 389

Bao, Z. Y., Käppeler, F. 1987, At. Data Nucl. Data Tables, 36, 411

Barkat, Z., \& Wheeler, J.C. 1990, ApJ, 355, 602

Branch, D., 1998, ARA\&A, 36, 17 
Branch, D., Doggett, J.B., Nomoto, K., \& Thielemann, F.-K. 1985, ApJ, 294, 619

Branch, D., Livio, M., Yungelson, L.R., Boffi, F.R., \& Baron, E. 1995, PASP, 107, 717

Branch, D., Tammann, G.A. 1992, ARA\&A 30, 359

Bravo, E., Isern, J., \& Canal, R. 1993, A\&A, 269, 187

Bravo, E., Isern, J., Canal, R., \& Labay, J. 1992, A\&A, 257, 534

Bychkov, V.V., \& Liberman, M.A., 1995a, ApJ, 451, 711

Canal, R., Ruiz-Lapuente, P., \& Isern, J. 1997, in Thermonuclear Supernovae, ed. P. Ruiz-Lapuente, R. Canal, \& J. Isern, (Dordrecht:Kluwer), 257

Cappellaro, E., Turatto, M., Tsvvetkov, D.Yu., Bartunov, O.S., Pollas, C., Evans, R. \& Hamuy, M. 1997, A\&A, 322, 431

Caughlan, G.R., \& Fowler, W.A. 1988, At. Data Nucl. Data Tables, 40,283

Caurier, E., Langanke, K., Martinez-Pinedo, G., Nowacki, F. 1999, Phys. Rev. C, submitted

Dean, D.J., Langanke, K., Chatterjee, L., Radha, P.B., \& Strayer, M.R. 1998, Phys. Rev. C, 58, 536

Di Stefano, R., Nelson, L.A., Lee, W., Wood, H., Rappaport, S. 1997, in Thermonuclear Supernovae, ed. P. Ruiz-Lapuente, R. Canal, \& J. Isern, (Dordrecht:Kluwer), 147

Fisher, A., Branch, D., Hö̈lich, P., \& Khokhlov, A. 1995, ApJ, 447, L73

Fuller, G.M., Fowler, W.A., \& Newman, M. 1980, ApJS, 42, 447

Fuller, G.M., Fowler, W.A., \& Newman, M. 1982, ApJS, 48, 279

Fuller, G.M., Fowler, W.A., \& Newman, M. 1985, ApJ, 293, 1

Gratton, R.G. 1989, A\&A, 208, 171

Gratton, R.G., \& Sneden, C. 1988, A\&A, 204, 193

Gratton, R.G., \& Sneden, C. 1991, A\&A, 241, 501

Hachisu, I., Kato, M., \& Nomoto, K. 1996, ApJ, 470, L97

Hachisu, I., Kato, M., \& Nomoto, K. 1999a, ApJ, 522, 487

Hachisu, I., Kato, M., Nomoto, K., \& Umeda, H. 1999b, ApJ, 519, 314

Hamuy, M., Phillips, M.M., Maza, J., Suntzeff, R.A., \& Aviles, R. 1995, AJ, 109,

Harkness, R.P. 1991, in SN 1987A and Other Supernovae, ed. I.J., Danziger \& K. Kjär (Garching:ESO), 447

Harper, C.L., Nyquist, L.H., Shih, C.-Y., Wiesmann, H. 1990, in Nuclei in the Cosmos, ed. H. Oberhummer, W. Hillebrandt (Garching, MPI für Physik und Astrophysik), MPA/P4, 138

Hartmann, D., Woosley, S.E., \& El Eid, M.F. 1985, ApJ, 297, 837

Hashimoto, M., Nomoto, K., \& Thielemann, F.-K. 1989, A\&A, 210, L5

Hashimoto, M. 1995, Prog. Theor. Phys., 94, 663

Hashimoto, M., Nomoto, K., Tsujimoto, T., \& Thielemann, F.K. 1996, in IAU Colloq. 145, Supernovae and Supernova Remnants, ed. R. McCray \& Z. Wang (Cambridge:Cambridge University Press), 157

Hatano, K., Branch, D., Fisher, A., Baron, E., \& Filippenko, A.V. 1999, ApJ Lett., submitted

Hillebrandt, W., \& Niemeyer, J.C. 1997, in Thermonuclear Supernovae, ed. P. Ruiz-Lapuente, R. Canal, \& J. Isern, (Dordrecht:Kluwer), 337

Hix, W.R., \& Thielemann F.-K. 1996, ApJ, 460, 869

Hix, W.R., \& Thielemann F.-K. 1999a, ApJ, 511, 862

Hix, W.R., \& Thielemann F.-K. 1999b, J. Climate Appl. Meteor., in press

Höflich, P., Dominguez, I., Thielemann, F.-K., \& Wheeler, J.C. 1999, in Proc. 42nd Int. Astrophys. Colloq. (ESA-SP$429 ;$ ESA:Noordwijk), 243

Höflich, P., \& Khokhlov, A. 1996, ApJ, 457, 500

Höflich, P., Wheeler, J.C., \& Thielemann, F.-K. 1998, ApJ, 495, 617

Hughes, J.P., et al. 1995, ApJ, 444, L81

Hwang, U., Hughes, J.P., \& Petre, R. 1998, ApJ, 497, 833

Iben, I.,Jr. 1982, ApJ, 253, 248

Iben, I.,Jr., \& Tutukov, A.V. 1984, ApJS, 54, 335

Ichimaru, S. 1993, Rev. Mod. Phys., 65, 255

Ireland, T.R. 1994, ApJ, 434, L79

Jeffery, D., Leibundgut, B., Kirshner, R.P., Benetti, S., Branch, D., \& Sonneborn, G. 1992, ApJ, 397, 304

Khokhlov, A.M. 1991a, A\&A, 245, 114

Khokhlov, A.M. 1991b, A\&A, 245, L25

Khokhlov, A.M. 1995, ApJ, 449, 695

Khokhlov, A.M., Müller, E., \& Höflich, P. 1992, A \& A, 253, L9

Khokhlov, A.M., Oran, E.S., \& Wheeler, J.C. 1997, ApJ, 478, 678

Kirshner, R.P., et al. 1993, ApJ, 415, 589

Kobayashi, C., Tsujimoto, T., Nomoto, K., Hachisu, I., \& Kato, M. 1998, ApJ, 503, L155

Landau, L.D., \& Lifshitz, E.M. 1987, Fluid Mechanics (Oxford:Pergamon)

Lee, T. 1988, in Meteorites and the Early Solar System, ed. J.F. Kerridge, \& M.S. Matthews (Tuscon:Univ. Arizona Press), 1063

Leibundgut, B., Kirshner R.P., Filippenko, A.V., Shields, J.C., Foltz, C.B., Phillips, M.M., \& Sonneborn, G. 1991a, ApJ, 371, L23
Lentz, E.J., Baron, E., Branch, D., Hauschildt, P.H., \& Nugent, P.E., 1999, ApJ, in press

Li, X.D., \& van den Heuvel, E.P.J. 1997, A\&A, 322, L9

Liu, W.H., Jeffery, D.J., \& Schultz, D.R. 1997, ApJ, 483, L107

Livne, E. 1993, ApJ, 406, L17

Livne, E., \& Arnett, W.D. 1995, ApJ, 452, 62

Loss, R.D., Lugmair, G.W. 1990, ApJ, 360, L59

Magain, P. 1987, A\&A, 179, 176

Magain, P. 1989, A\&A, 209, 211

Matteucci, F., Raiteri, C.M., Busso, M., Gallino, R., \& Gratton, R. 1993, A\&A, 272, 421

Mazzali, P., Cappelaro, E., Danziger, I.J., Turatto, M., \& Benetti, S. 1998, ApJ, 499, L49

Mazzali, P., Lucy, L.B., Danziger, I.J., Gouiffes, C., Cappellaro, E.R., \& Turatto, M. 1993, A\&A, 269, 423

Meikle, W.P.W., et al. 1996, MNRAS, 281, 263

Meyer, B.S., Krishnan, T.D., \& Clayton, D.D. 1996, ApJ, 462, 825

Miyata, E., Tsunemi, H., Kohmura, T., Suzuki, S., \& Kumagai, S. 1998, PASJ, 50, 257

Nakamura, T., Umeda, H., Nomoto, K., Thielemann, F.-K., \& Burrows, A. 1999, ApJ, 517, 193

Niemeyer, J.C. 1999, ApJ, 523, L57

Niemeyer, J.C., \& Hillebrandt W. 1995a, ApJ, 452, 769

Niemeyer, J.C., Hillebrandt W., \& Woosley, S.E. 1996, ApJ, 471, 903

Niemeyer, J.C., \& Kerstein, A.R. 1997, NewA, 2, 239

Niemeyer, J.C., \& Woosley, S.E. 1997, ApJ, 475, 740

Nissen, P.E., Gustafsson, B., Edvardsson, B., \& Gilmore, G. 1994, A\&A, 285, 440

Nomoto, K. 1982a, ApJ, 253, 798

Nomoto, K. 1982b, ApJ, 257, 780

Nomoto, K., \& Hashimoto, M. 1988, Phys. Rep., 163, 13

Nomoto, K., \& Iben, I.,Jr. 1985, ApJ, 297, 531

Nomoto, K., Iwamoto, K., \& Kishimoto, N. 1997, Science, 276, 1378

Nomoto, K., Hashimoto, M., Tsujimoto, T., Thielemann, F.-K. Kishimoto, N., Kubo, Y., \& Nakasato, N. 1997a, Nucl. Phys. A, $161,79 \mathrm{c}$

Nomoto, K., Iwamoto, K., Nakasato, N., Thielemann., F.K., Brachwitz, F., Tsujimoto, T., Kubo. Y., \& Kishimoto, N. 1997b, Nucl. Phys. A, 621, 467c

Nomoto, K., et al. 1997c, in Thermonuclear Supernovae, ed. P. RuizLapuente, R. Canal, \& J. Isern, (Dordrecht:Kluwer), 349

Nomoto, K., \& Kondo, Y. 1991, ApJ, 367, L19

Nomoto, K., Nariai, K., \& Sugimoto, D. 1979, PASJ, 31, 287

Nomoto, K., Sugimoto, D., \& Neo, S. 1976, Ap\&SS, 39, L37

Nomoto, K., Thielemann, F.-K., \& Yokoi, K. 1984, ApJ, 286, 644

Nomoto, K., Yamaoka, H., Shigeyama, T., Kumagai, S., \& Tsujimoto, T. 1994, in Les Houches, Session LIV, Supernovae, ed. S. Bludman, R.Mochovitch, \& Zinn-Justin(Amsterdam:Elsevier), 199

Nomoto, K., Yamaoka, H., Shigeyama, T., \& Iwamoto, K. 1996a, in IAU Colloq. 145, Supernovae and Supernova Remnants, ed. R. McCray \& Z. Wang (Cambridge:Cambridge University Press), 49

Nugent, P., Baron, E., Branch, D., Fisher, A., \& Hauschildt, P.H. 1997, ApJ, 485, 812

Paczyński, B. 1973, Acta Astron, 23, 1

Pagel, B.E.J., \& Trautvaisiene, G. 1995, MNRAS, 276, 505

Pagel, B.E.J., \& Trautvaisiene, G. 1997, MNRAS, 288, 108

Perlmutter, S., et al. 1997, ApJ, 483, 565

Perlmutter, S., et al. 1999, ApJ, 517, 565

Phillips, M.M., Hamuy, M., Maza, J., Ruiz, M.T., Carney, B.W., \& Graham, J.A. 1990, PASP, 102, 299

Rappaport, S., Di Stefano, R., \& Smith, J.D., 1994 ApJ, 426, 692

Rauscher, T., Applegate, J.H., Cowan, J.J., Thielemann, F.-K., \& Wiescher, M. 1994, ApJ, 429, 499

Renzini, A. 1996, in IAU Colloq. 145, Supernovae and Supernova Remnants, ed. R. McCray \& Z. Wang (Cambridge:Cambridge University Press), 77

Riess, A.G., Press, W.H., \& Kirshner, R.P. 1995, ApJ, 438, L17

Riess, A.G., et al. 1999, AJ, 117, 707

Ruiz-Lapuente, P. 1997, in Thermonuclear Supernovae, ed. P. RuizLapuente, R. Canal, \& J. Isern, (Dordrecht:Kluwer), 681

Ryan, S.G., Norris, J.E., \& Beers, T.C. 1996, ApJ, 471, 254

Saio, H., \& Nomoto, K. 1985, A\&A, 150, L21

Saio, H., \& Nomoto, K. 1998, ApJ, 500, 388

Shigeyama, T., Nomoto, K., Yamaoka, H., \& Thielemann, F.-K. 1992, ApJ, 386, L13

Thielemann, F.-K., Arnould, M., \& Truran, J. W. 1987, in Advances in Nuclear Astrophysics, ed. E. Vangioni-Flam (Gif sur Yvette:Editions Frontière), 525

Thielemann, F.-K., Nomoto, K., \& Hashimoto, M. 1996, ApJ, 460, 408

Thielemann, F.-K., Nomoto, K., Iwamoto, K., \& Brachwitz, F. 1997, in Thermonuclear Supernovae, ed. P. Ruiz-Lapuente, R. Canal, \& J. Isern (Dordrecht:Kluwer), 485

Thielemann, F.-K., Nomoto, K., \& Yokoi, K. 1986, A\&A, 158, 17 
Timmes, F., \& Woosley, S.E. 1992, ApJ, 396, 649

Timmes, F., Woosley, S.E., \& Weaver, T.A. 1995, ApJS, 98, 617

Travaglio, C., Gallino, R., Amarari, S., Zinner, E., Woosley, S.E., \& Lewis, R. 1999, ApJ, 510, 325

Trimble, V. 1975, Rev. Mod. Phys., 47, 877

Tsujimoto, T., Nomoto, K., Yoshii, Y., Hashimoto, M., Yanagida, S., \& Thielemann, F.-K. 1995, MNRAS, 277, 945

Umeda, H., Nomoto, K., Yamaoka, H, \& Wanajo, S. 1999, ApJ, 513, 861

Unno, W. 1968, PASJ, 19, 140

van den Bergh, S., \& Tammann, G. 1991, ARA\&A, 29, 363

van den Heuvel, E.P.J., Bhattacharya, D., Nomoto, K., \& Rappaport, S.A. 1992, A\&A, 262, 97

van Wormer, L., Görres, J., Iliadis, C., Wiescher, M., \& Thielemann, F.-K. 1994, ApJ, 432, 267

Völkening, J. \& Papanastassiou, D.A. 1989, ApJ, 347, L43

Völkening, J. \& Papanastassiou, D.A. 1990, Lunar and Planetary Science XXI(Houston:Lunar.Planet.Inst.), No 1276

Webbink, R.F. 1984, ApJ, 277, 355
Wheeler, J.C., Harkness, R.P., Khokhlov, A.M., \& Höflich, P. 1995, Phys. Rep., 53, 221

Woosley, S.E. 1997a, in Thermonuclear Supernovae, ed. P. RuizLapuente, R. Canal, \& J. Isern (Dordrecht:Kluwer), 313

Woosley, S.E. 1997b, ApJ, 476, 801

Woosley, S.E., \& Weaver, T.A. 1986, ARA\&A, 24, 205

Woosley, S.E., \& Weaver, T.A. 1994a, ApJ, 423, 371

Woosley, S.E., \& Weaver, T.A. 1994b, in Les Houches, Session LIV, Supernovae, ed. S.A.Bludman, R.Mochovitch, \& J.ZinnJustin(Amsterdam:Elsevier), 63

Yamaoka, H., Shigeyama, T., Nomoto, K., \& Thielemann, F.-K. 1992, ApJ, 393, L55

Yoshii, Y., Tsujimoto, T., \& Nomoto, K. 1996, ApJ, 462, 266

Zhao, G., \& Magain, P. 1990, A\&A 238, 242

Zinner, E. 1995, in Nuclei in the Cosmos III, ed. M. Busso, R. Gallino, \& C.M. Raiteri (New York:AIP Press), 567

Zinner, E., Tang, M., \& Anders, E. 1989, Geochim. Cosmochim. Acta, 53,3273 

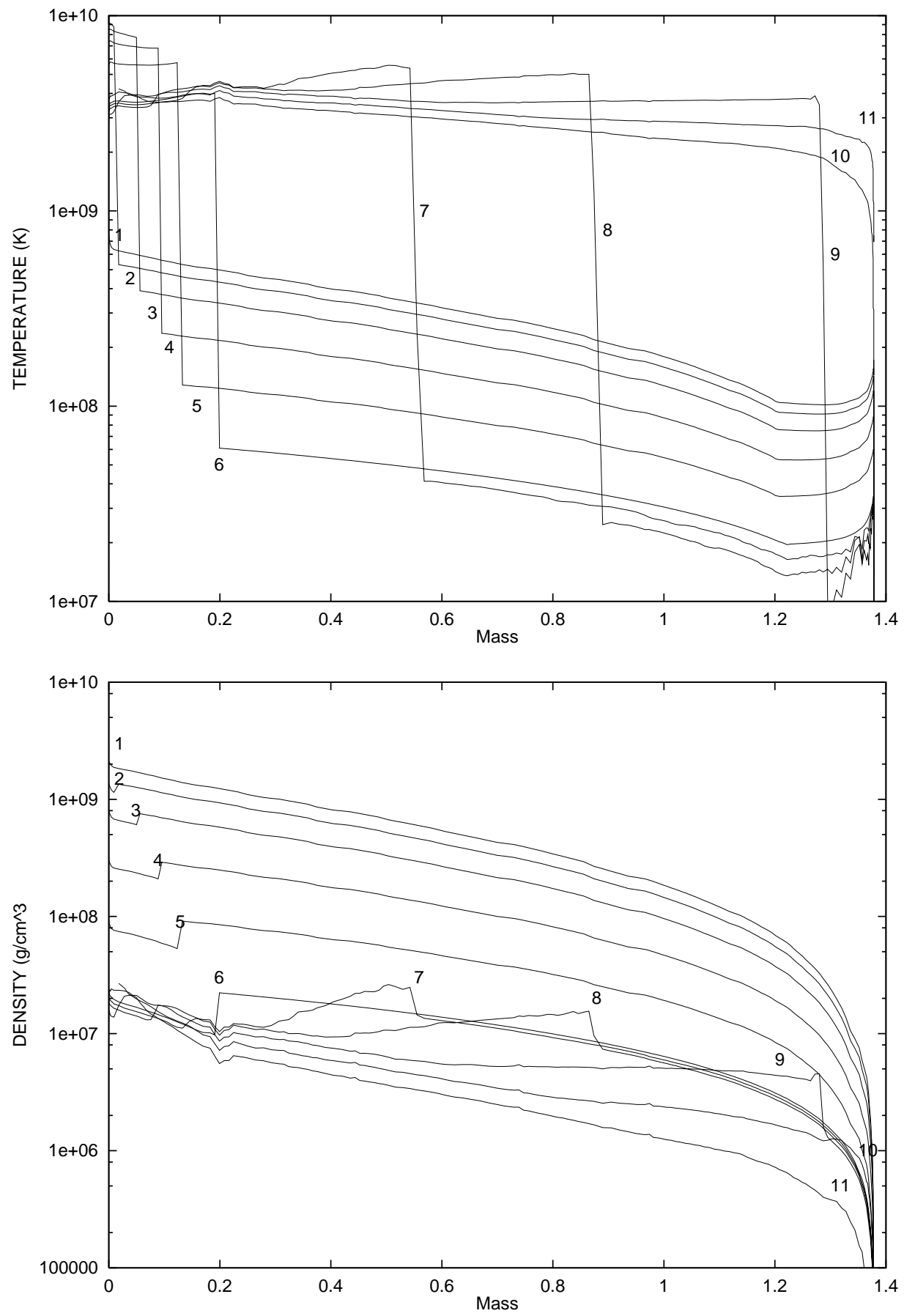

Fig. 1. - Temperatures and densities for the deflagration wave (WS15) and delayed detonation (DD1) as a function of radial mass coordinate $M_{r}$. The labels indicate different instances in time, 1-6 correspond to the deflagration phase, 7-11 to the propagation of the detonation after the deflagration-detonation transition. 

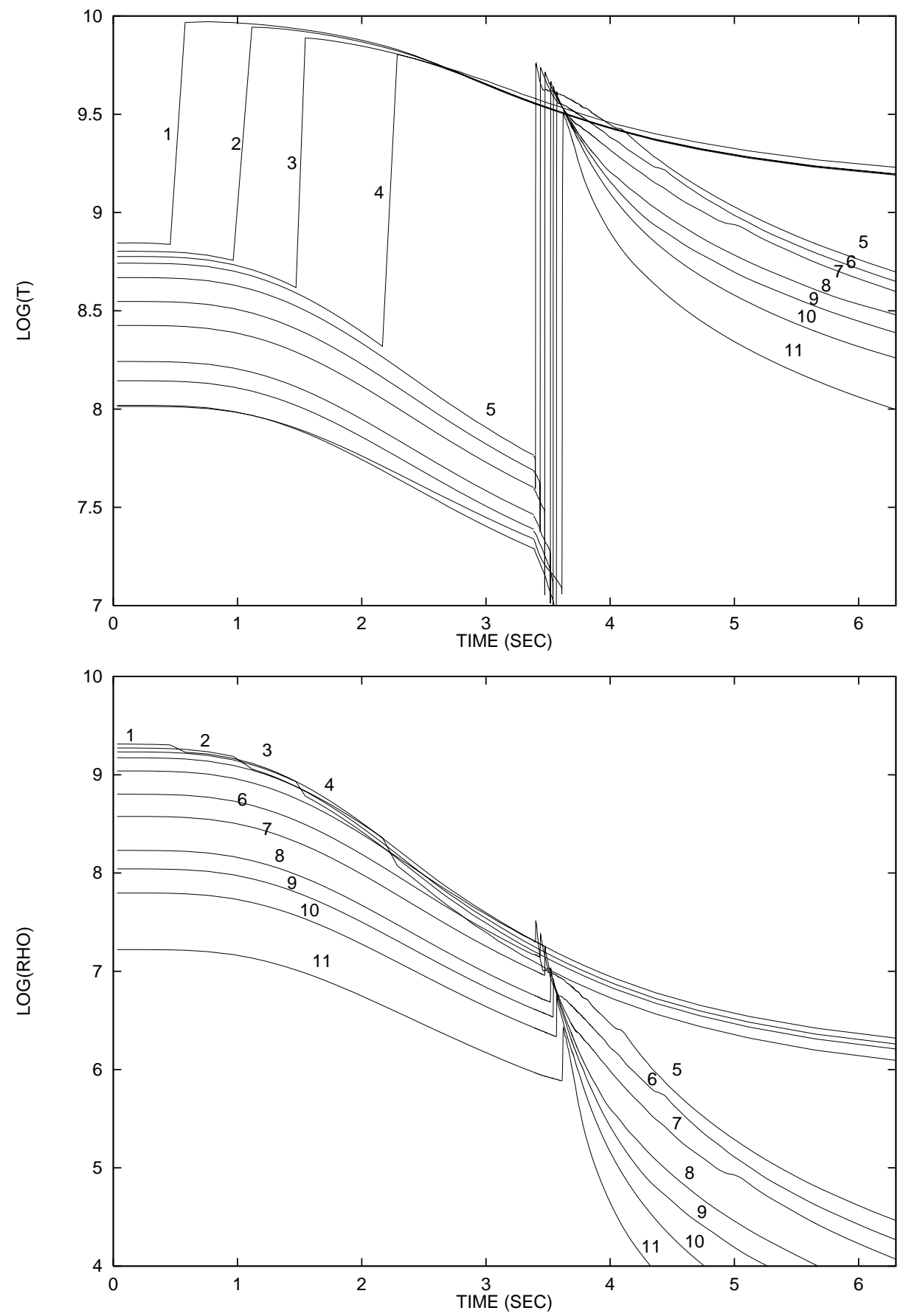

FIG. 2.- The distribution of temperatures and densities as a function of time during the passage of the slow deflagration (WS15) and the subsequent delayed detonation (DD1). The labeled numbers indicate different mass zones. The prominent spike, occurring for labels $\geq 5$, shows the action of the detonation and its fast propagation (small time differences between different mass zones). 


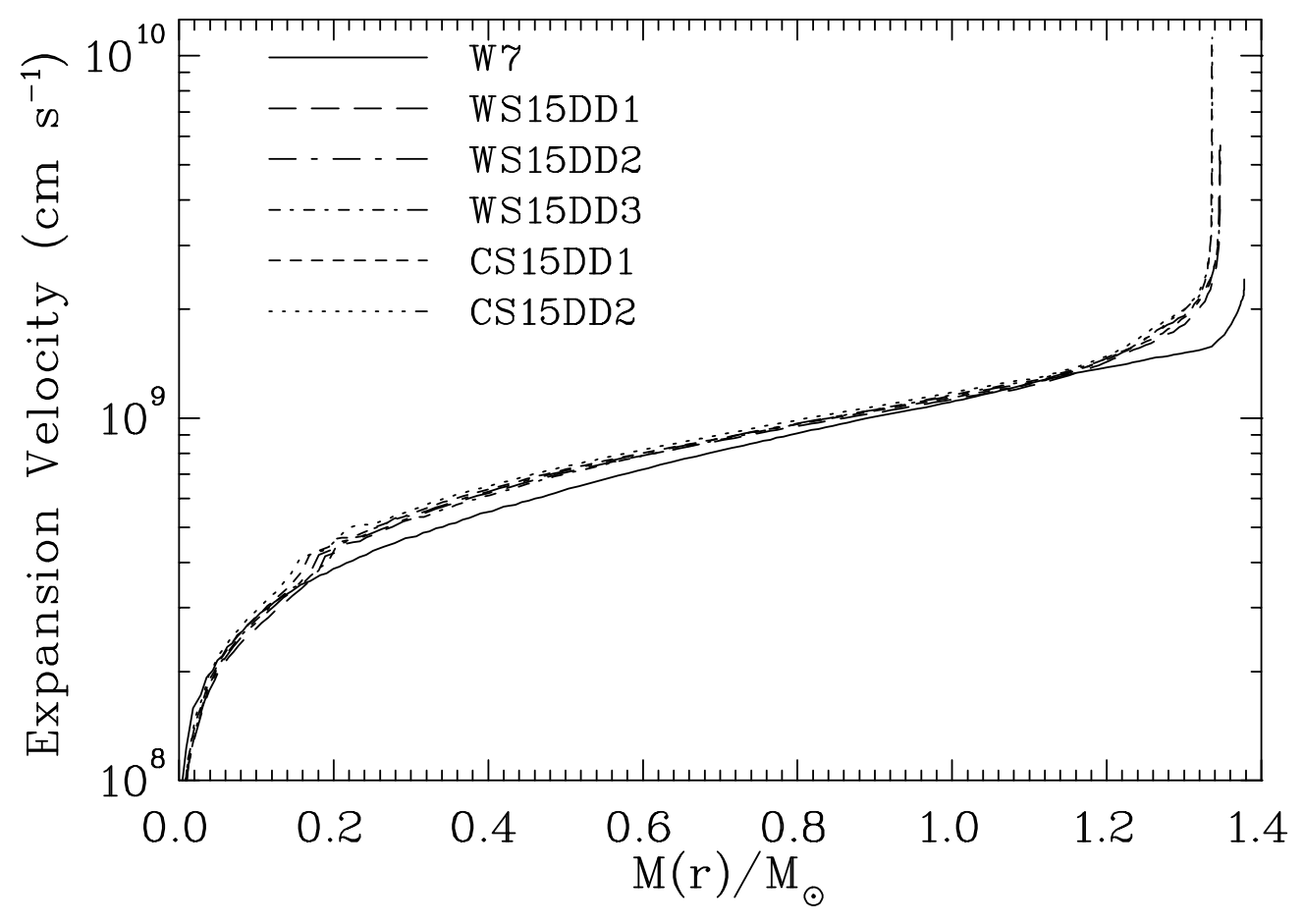

Fig. 3.- The distribution of expansion velocities after the passage of the slow deflagration and the subsequent delayed detonation. 


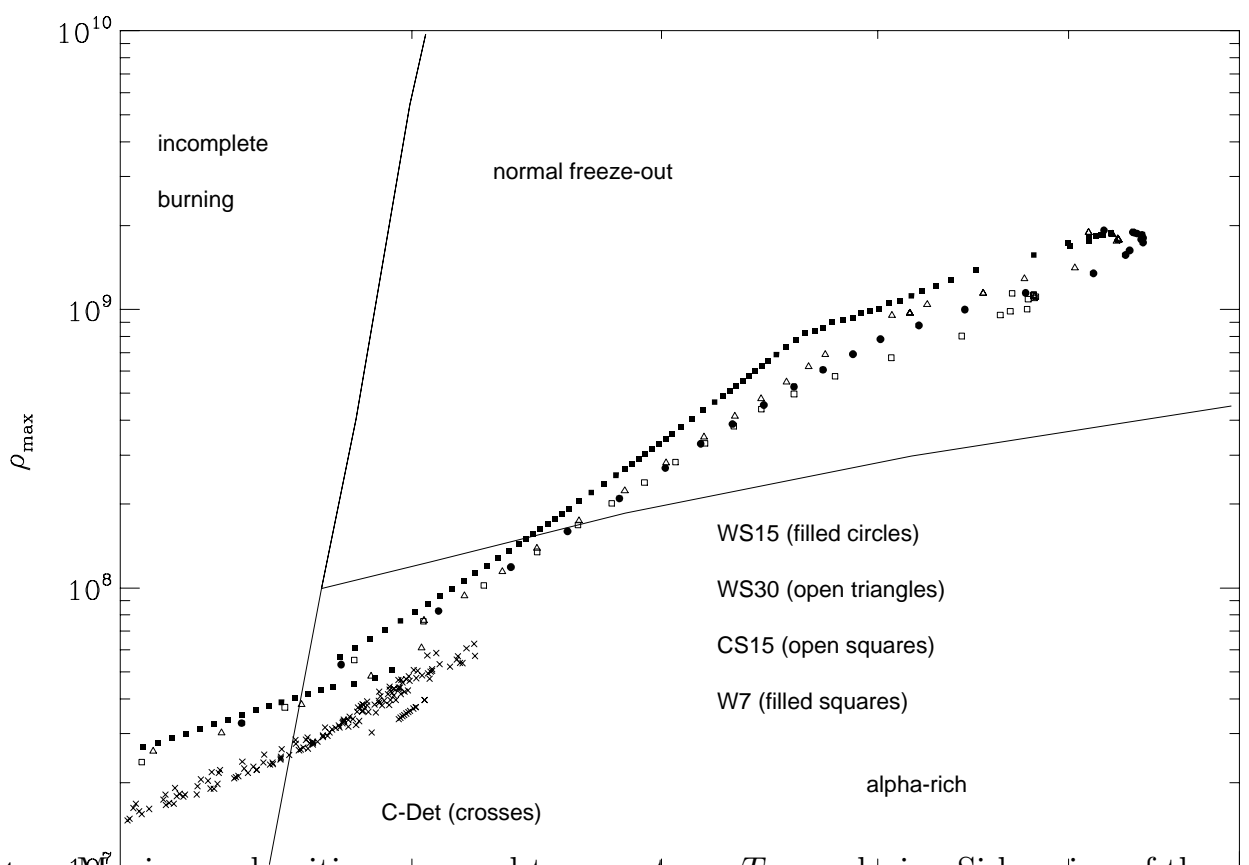

Fig. 4.- 1DFiaximum densities $\rho_{\max }$ and temperatures $T_{9 \text {,max }}$ during Si-burning of the central layers obtained during the propagation of the burning front. The model $\mathrm{W}_{\mathrm{T}}$ is compared to three slow deflagrations which start (for a density of $2.1 \times 10^{9} \mathrm{~g} \mathrm{~cm}^{-3}$ ) with burning front velocities of $1.5 \%$ (slow deflagration WS15) and 3\% (WS30) of the sound speed and for an ignition density of $1.37 \times 10^{9} \mathrm{~g} \mathrm{~cm}^{-3}$ with a burning front velocity of $1.5 \%$ of sound (CS15). The crosses (C-det) correspond to carbon detonations of sub-Chandrasekhar mass models. They will not be discussed here, but it is obvious that the smaller temperatures and densities lead to negligible amounts of electron captures.

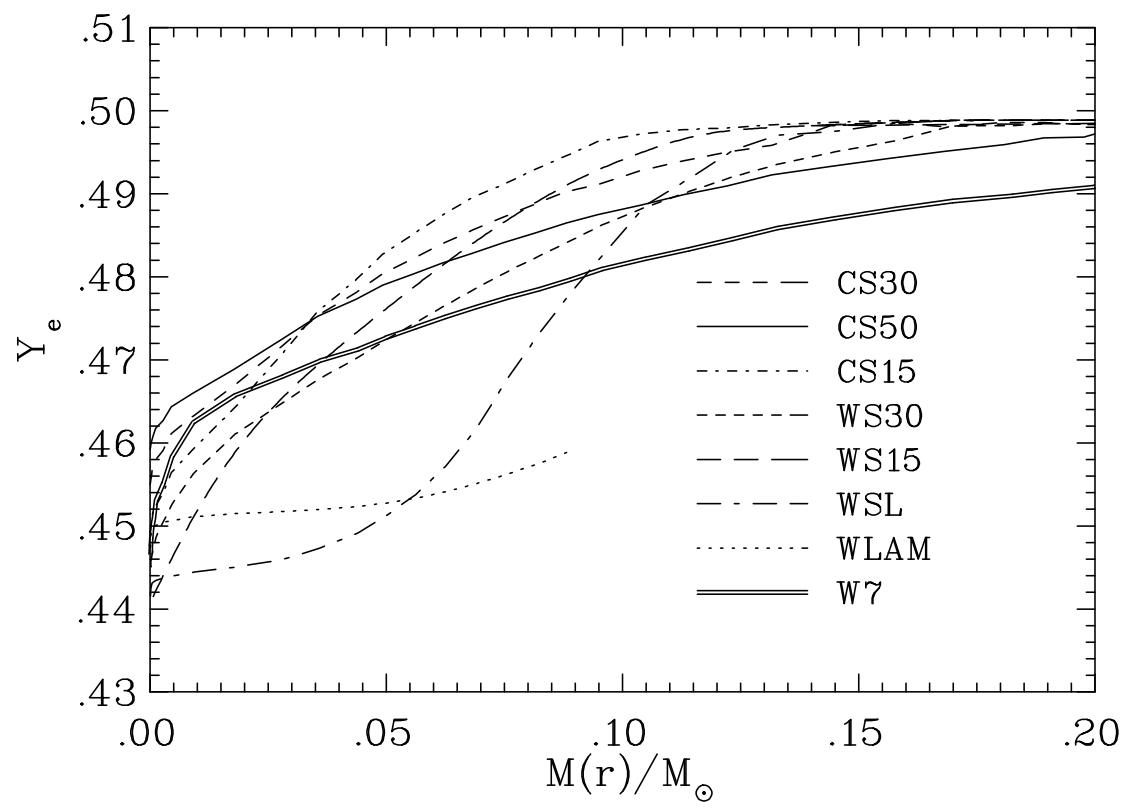

Fig. 5.- $Y_{e}$, the total proton to nucleon ratio and thus a measure of electron captures on free protons and nuclei, after freeze-out of nuclear reactions as a function of radial mass for different models. In general it can be recognized that small burning front velocities lead to steep $Y_{e}$-gradient which flatten with increasing velocities (see the series of models CS15, CS30, and CS50 or WS15, WS30, and W7). Lower central ignition densities shift the curves up (C vs. W), but the gradient is the same for the same propagation speed. This only changes when the $Y_{e}$ attained via electron captures during explosive burning is smaller than for stable Fe-group nuclei. Then, $\beta^{-}$-decay during the expansion to smaller temperatures and densities will reverse this effect (see models WSL and WLAM discussed in more detail in the text). 


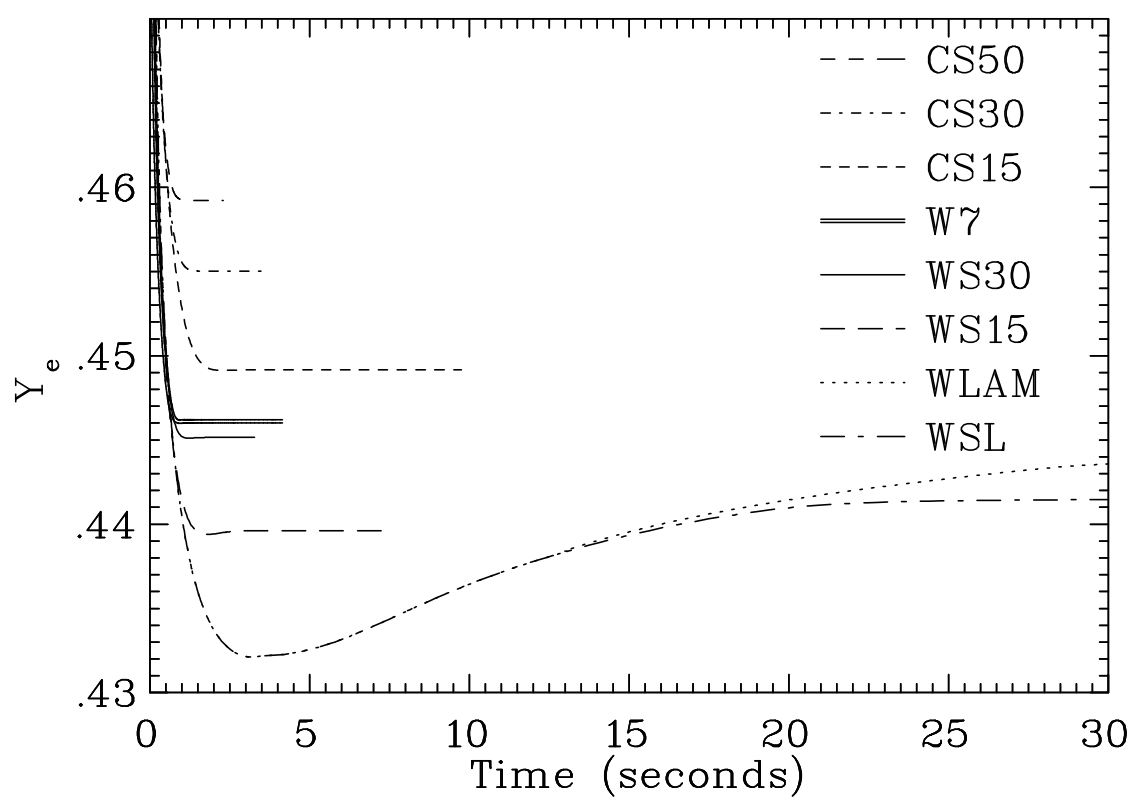

FIG. 6. $-Y_{e}(t)$ for the central mass zones in CS, WS, WSL, WSLAM and W7 models. We see that $Y_{e}$ attains the lowest values for the highest ignition densities and slowest burning front speeds. This tendency continues for the extreme cases of laminar burning front models, but the slow expansion in these models permits fast $\beta^{-}$-decays of short-lived nuclei in NSE or QSE equilibria before charged-particle freeze-out.

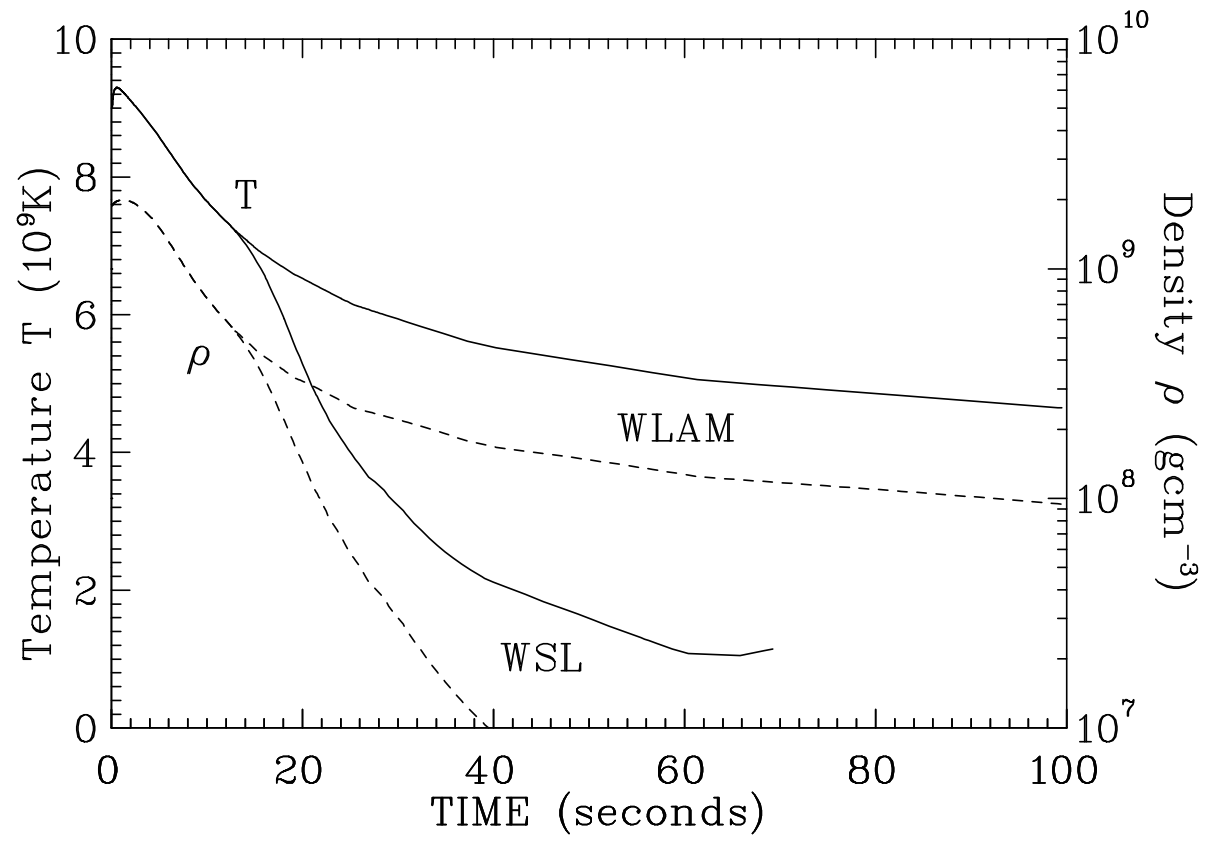

FIG. 7.- $\rho(t)$ and $T(t)$ for the central mass zones in WSL and WSLAM models. The bifurctaion between WSL and WLAM marks the point where the burning front was accelerated beyond the laminar speed in WSL, resulting in a faster expansion. 

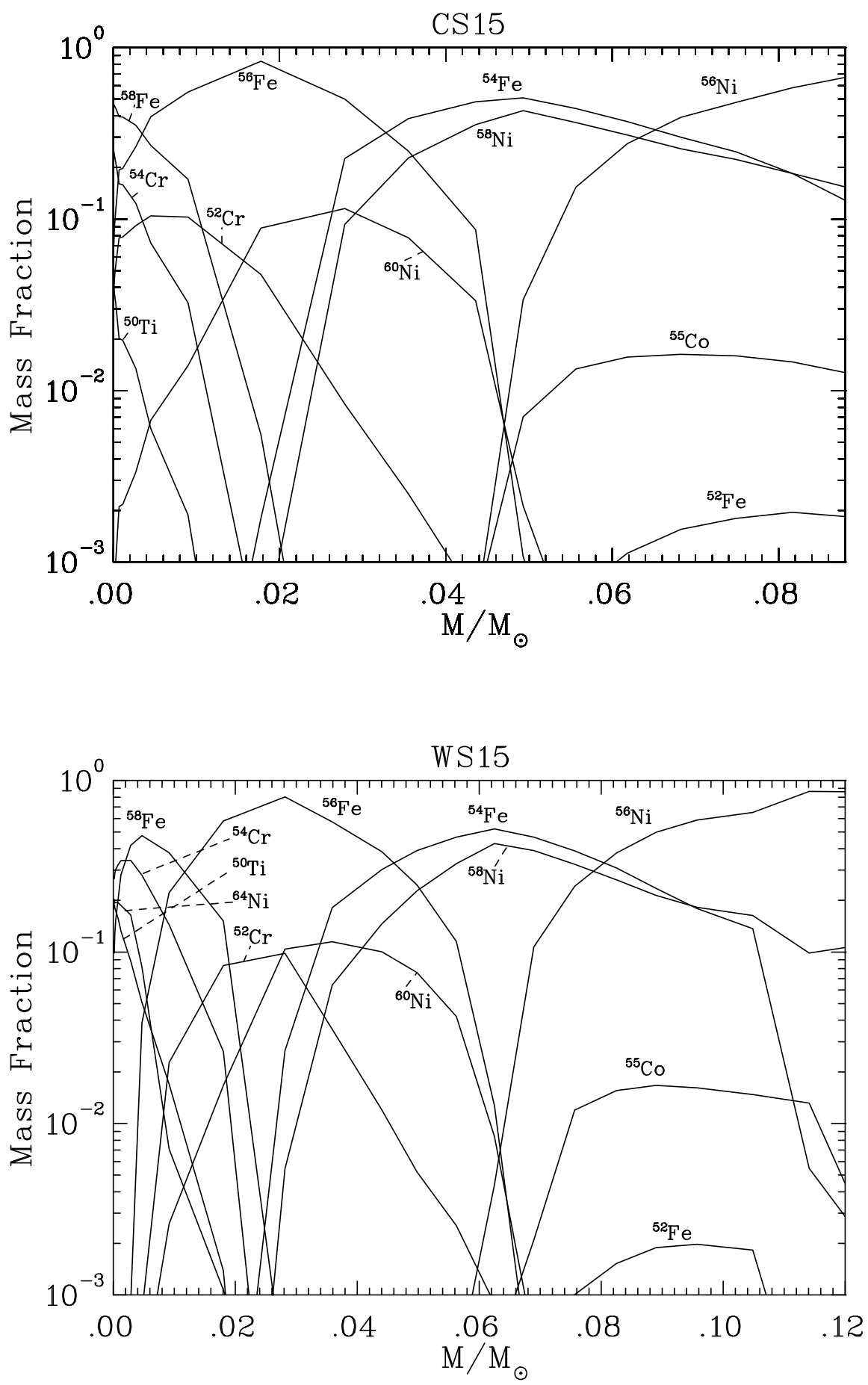

Fig. 8. - Abundance plots (mostly of Fe-group nuclei) for the cases CS15 and WS15. Shown are the locations of the nuclei overproduced in $\mathrm{W} 7,{ }^{54} \mathrm{Fe}$ and ${ }^{58} \mathrm{Ni}$, corresponding to $Y_{e}$ values of $0.47-0.485$. Due to the $Y_{e}$-gradients which are steeper than for $\mathrm{W} 7$, the amount of matter in a given $Y_{e}$-range is reduced, but also smaller central values are attained, giving rise to more neutron-rich nuclei. A $Y_{e}$ of about $0.46 \approx 26 / 56$ (which was also attained in W7) causes no problems and leads to a large abundance of stable ${ }^{56} \mathrm{Fe}$ (not from ${ }^{56} \mathrm{Ni}$ decay). Values in the range of 0.44 to 0.46 result also in ${ }^{50} \mathrm{Ti}$, ${ }^{54} \mathrm{Cr},{ }^{58} \mathrm{Fe}$, and ${ }^{62,64} \mathrm{Ni} .{ }^{48} \mathrm{Ca}$ with $\mathrm{Z} / \mathrm{A} \approx 0.42$ is only produced if $Y_{e}$ approaches values smaller than 0.44 (see Hartmann et al. 1985). 

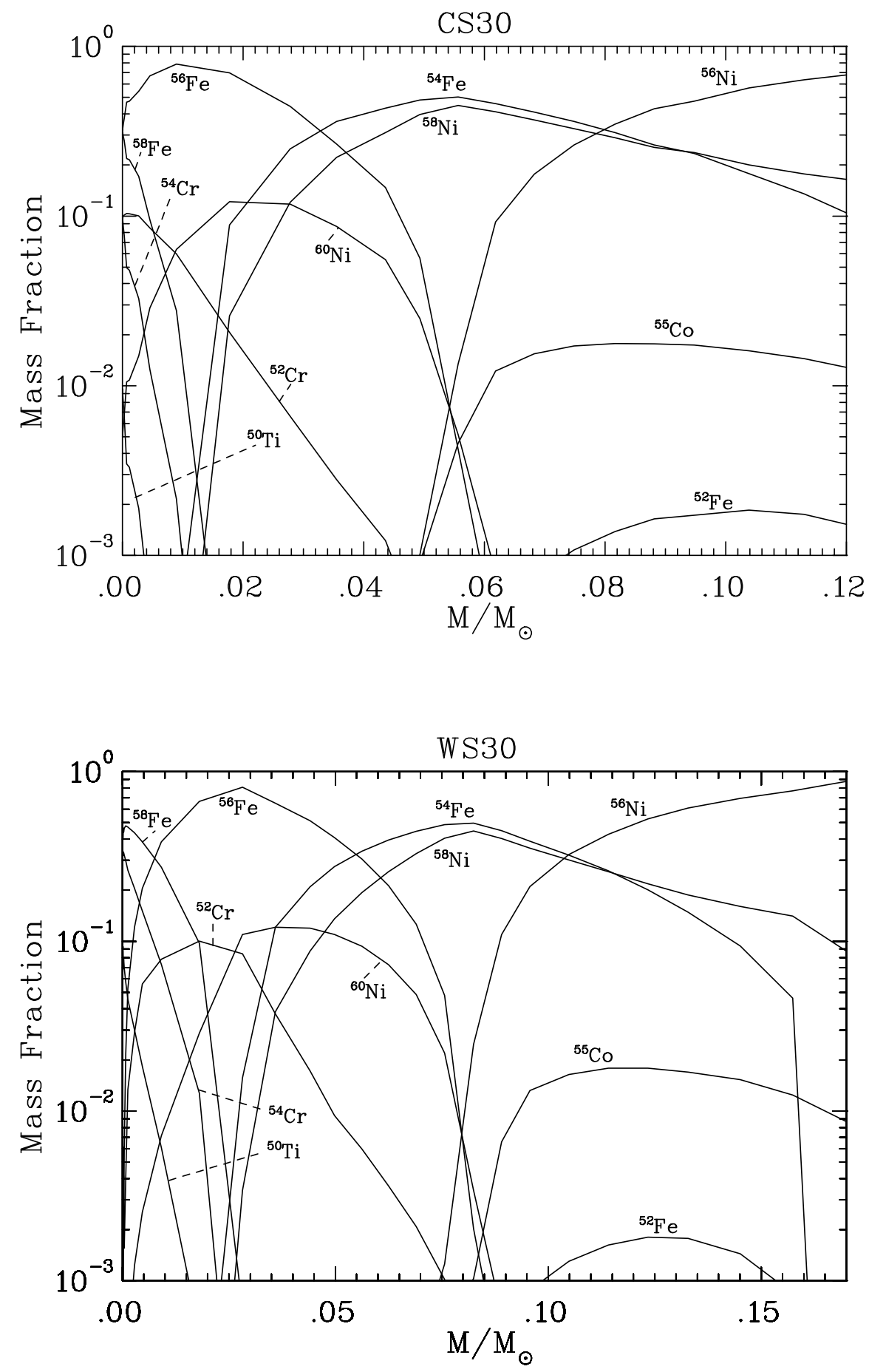

Fig. 9.- Abundance plots (mostly of Fe-group nuclei) for the cases Same as 8 for the cases CS30 and WS30. 

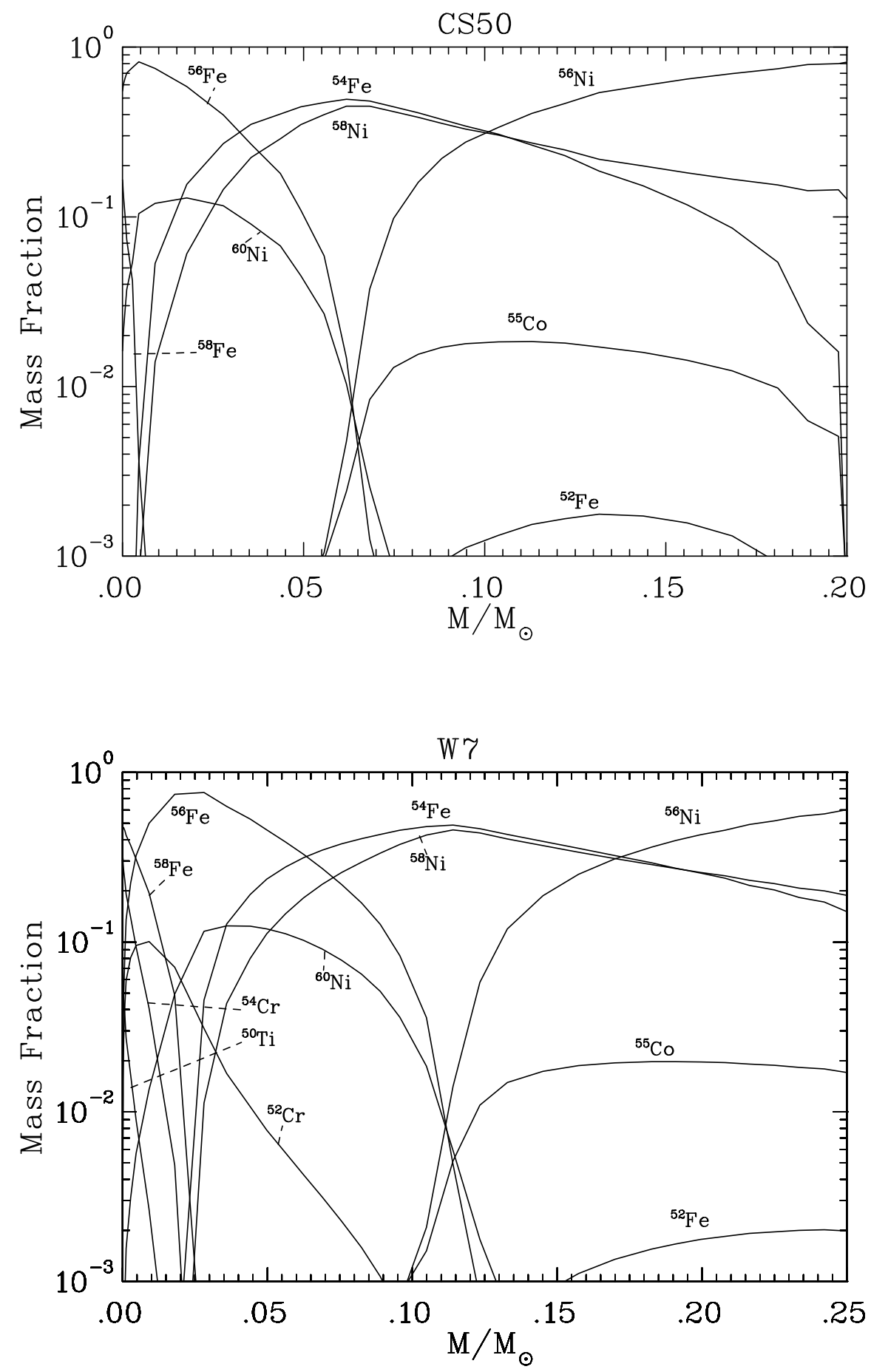

FIG. 10.- Same as 8 for the cases CS50 and W7. One sees that the low $Y_{e}$ region becomes more extended with increasing burning front velocities, while the central values increase somewhat. ${ }^{55} \mathrm{Co}$ and ${ }^{52} \mathrm{Fe}$ are not plotted here for W7 but are are shown in Figure 14. 
WSL

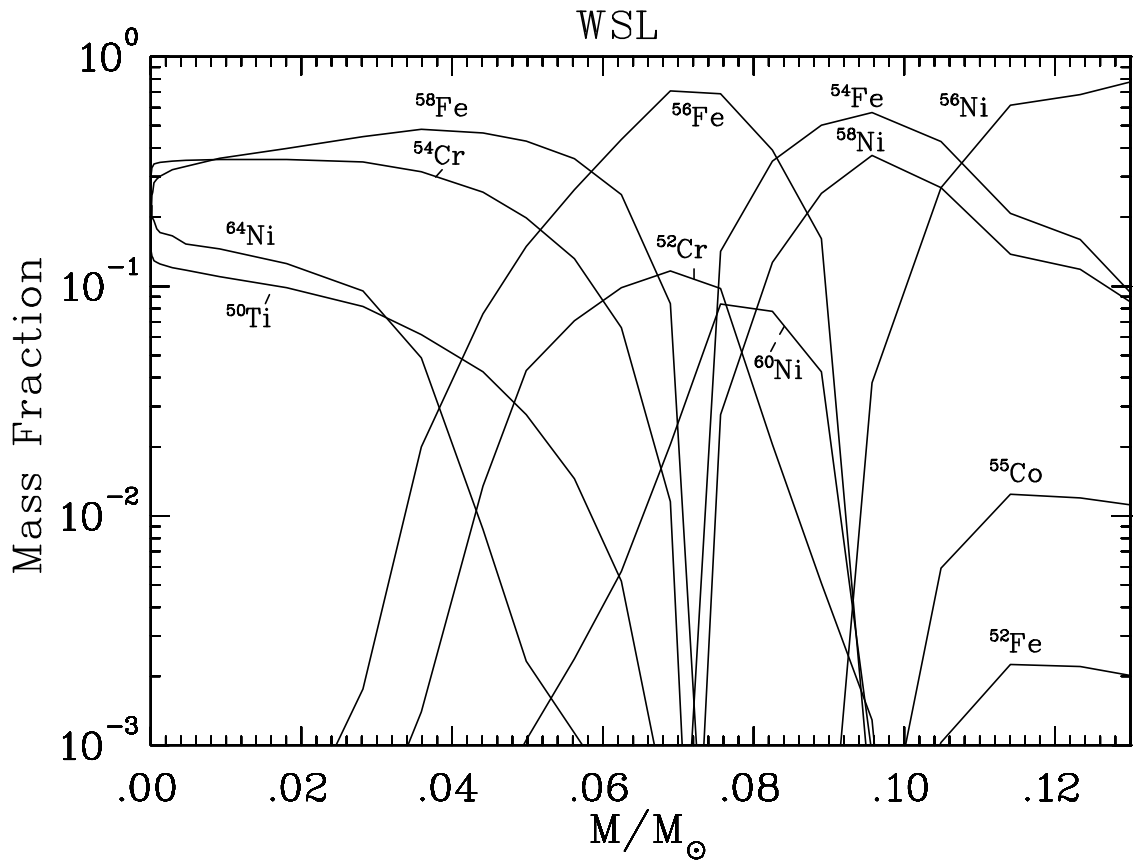

WLAM

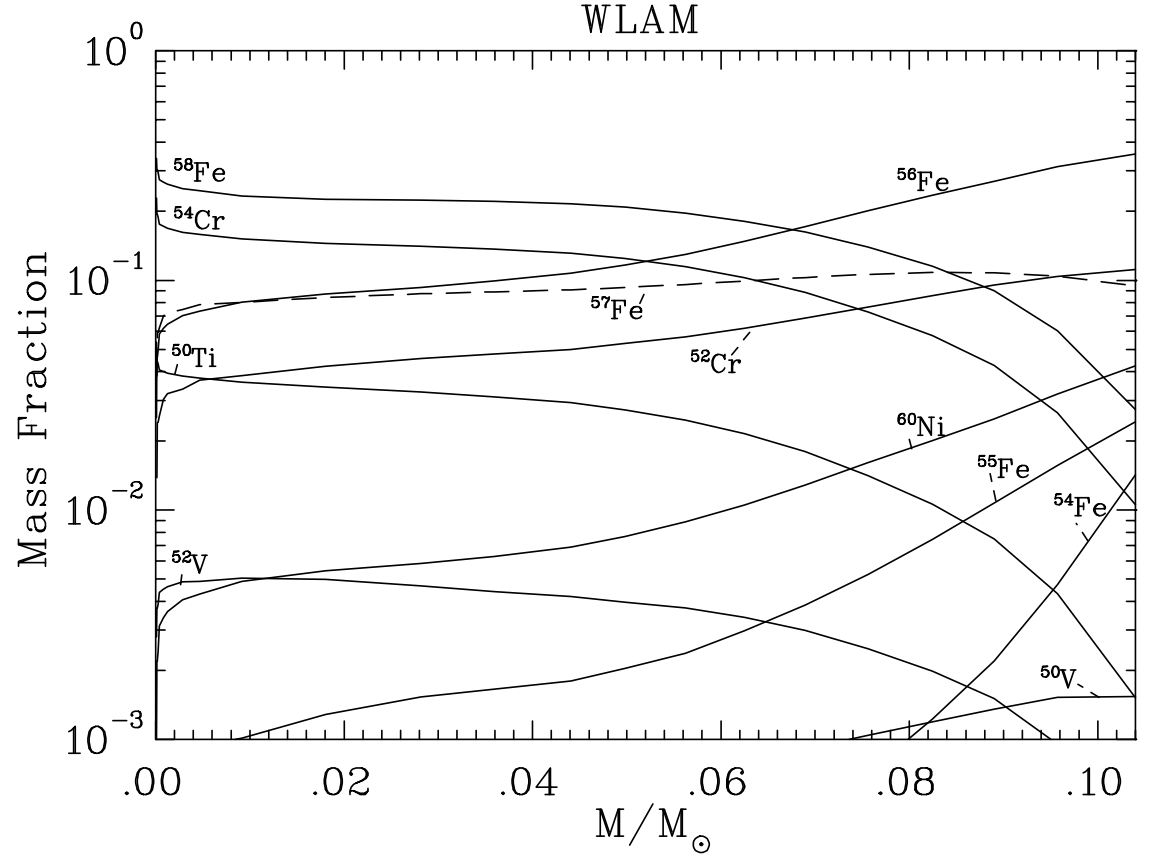

Fig. 11. - Same as 8 for the cases WSL and WLAM. WLAM has an almost constant $Y_{e}\left(M_{r}\right)$ with values close to those of WSL at $0.05 \mathrm{M}_{\odot}$ (see also Figure 5). 


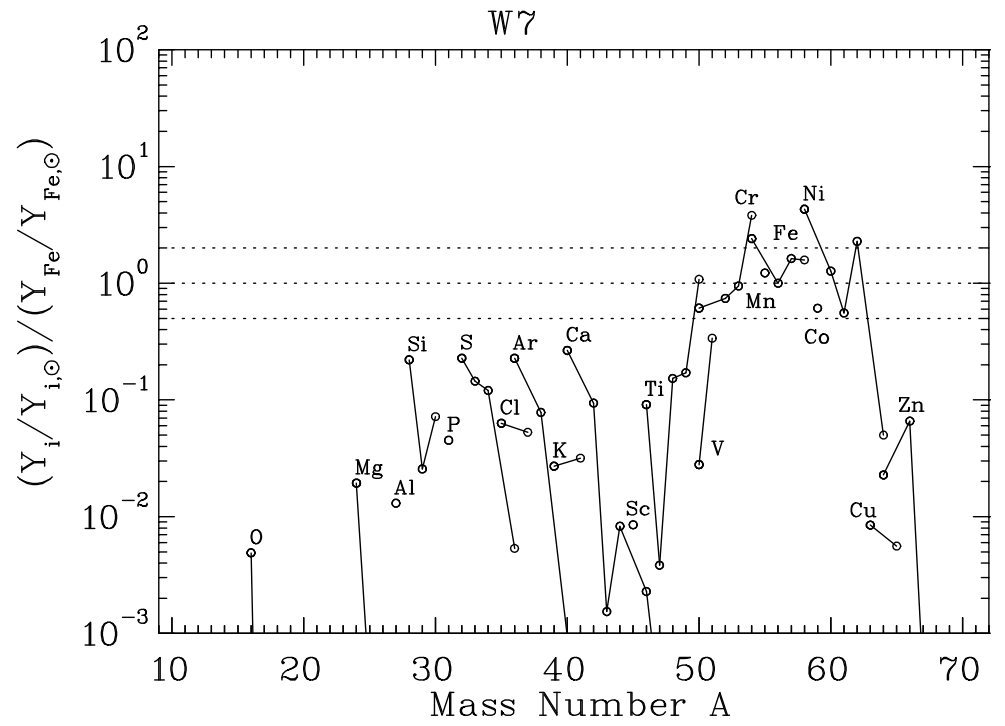

FIG. 12.- Ratio of abundances to solar predicted in model W7 (this is a recalculation of the 1986 model [Thielemann, Nomoto \& Yokoi 1986] with presently available updated reaction rates and a screened NSE treatment for temperatures beyond $6 \times 10^{9} \mathrm{~K}$, as described in Hix \& Thielemann 1996). Isotopes of one element are connected by lines. The ordinate is normalized to ${ }^{56} \mathrm{Fe}$. Intermediate mass elements exist, but are underproduced by a factor of 2-3 in comparison to Fe-group elements. Among the Fe-group, ${ }^{54} \mathrm{Cr}$ and ${ }^{58} \mathrm{Ni}$ are overproduced by a factor of 4 , which exceeds the permitted factor of $\sim 3$.

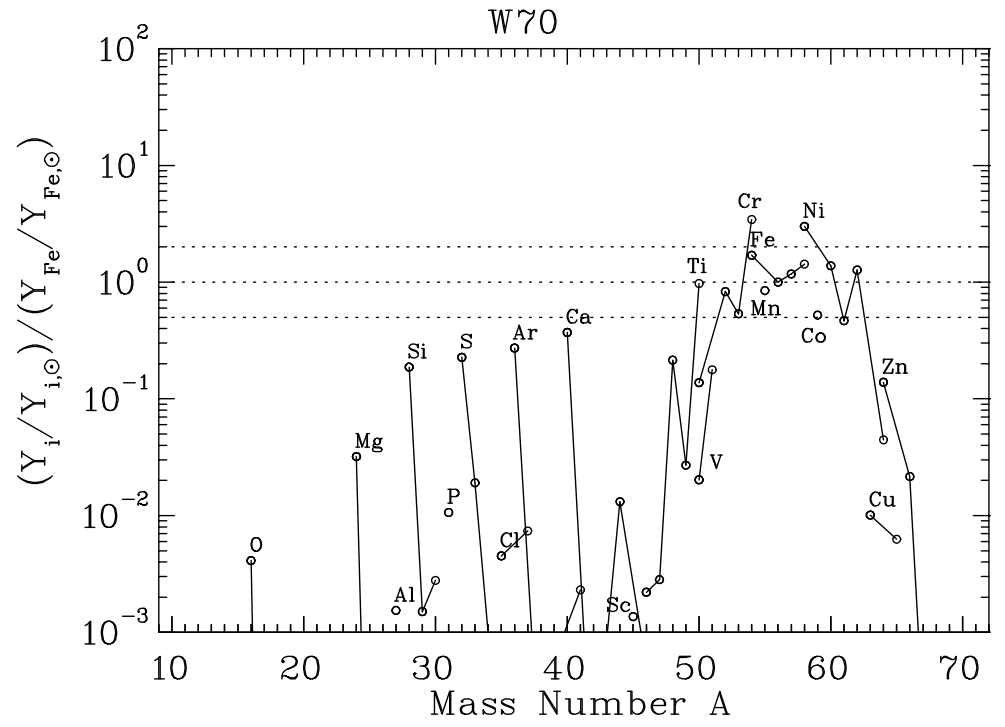

FIg. 13.- Same as Figure 12, i.e. a deflagration model treated exactly like W7, but with an initial composition corresponding to zero metallicity $\left(50 \%\right.$ mass fractions of ${ }^{12} \mathrm{C}$ and ${ }^{16} \mathrm{O}$ without ${ }^{22} \mathrm{Ne}$ admixture, i.e. $\left.Y_{e}=0.5\right)$. The production of neutron-rich species ${ }^{54} \mathrm{Fe},{ }^{58} \mathrm{Ni}$, and ${ }^{62} \mathrm{Ni}$ are smaller than the original $\mathrm{W} 7$ model. Noticeable is also the almost complete absence of intermediate odd-Z elements which are produced in explosive oxygen burning for a $Y_{e}$ smaller than 0.5. The even- $Z$ intermediate mass elements are also effected. They are not set off by a constant factor in comparison to ${ }^{56} \mathrm{Fe}$, as seen in Figure 12 for $\mathrm{W} 7$, but increase with mass. The increasing deviation between $N=Z$ and a line in the nuclear chart with $Y_{e}<0.5$, with increasing nuclear mass, does not occur. 

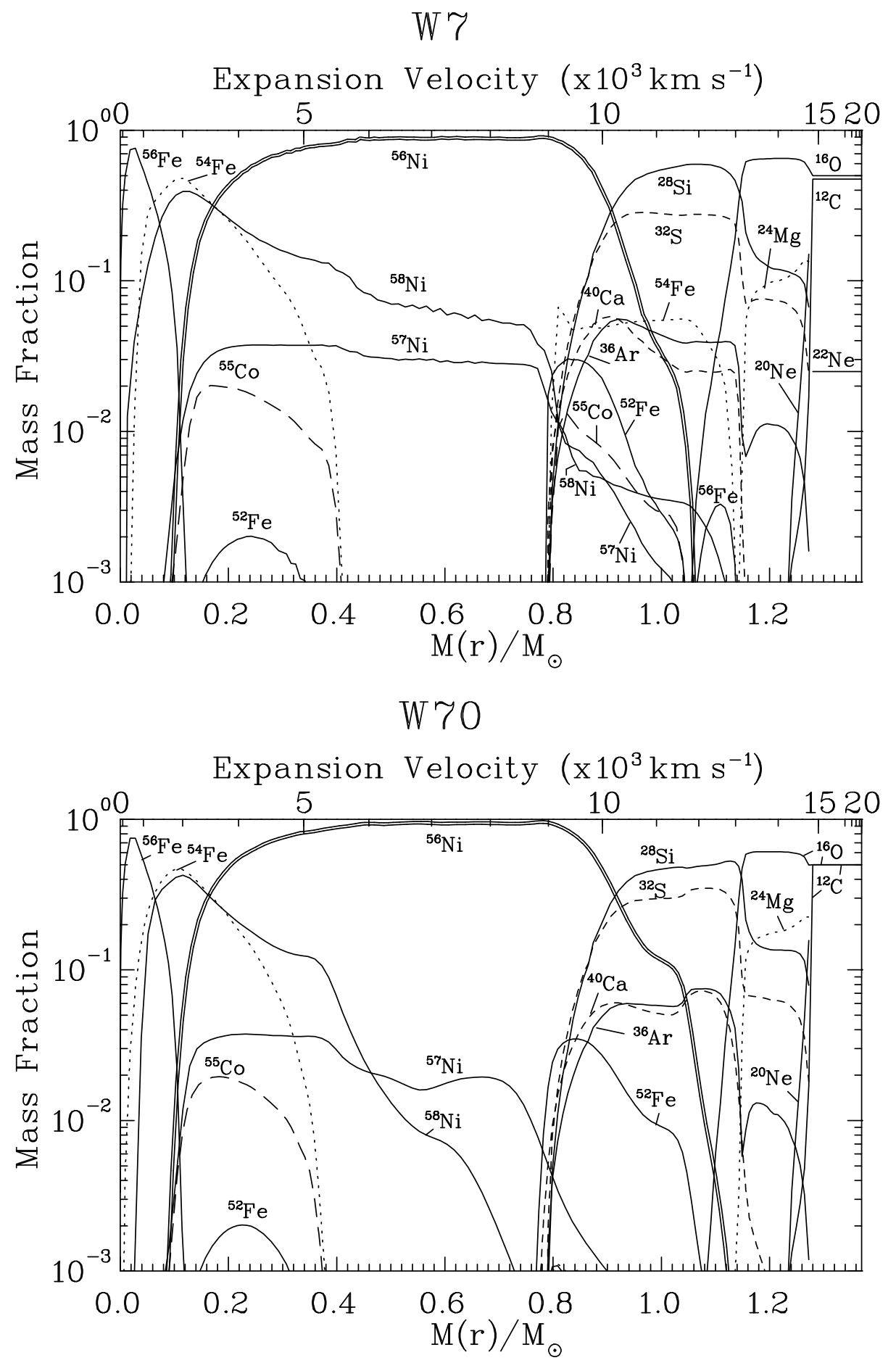

FIG. 14.- Composition of $\mathrm{W} 7$ and $\mathrm{W} 70$. The major changes are given in the mass zones outside the central $0.3 \mathrm{M} \odot$, where $Y_{e}$ is enherited from the initial metallicity in form of ${ }^{22} \mathrm{Ne}$ and not due to electron captures. This has effects on the Fe-group composition in the alpha-rich freeze-out region (on ${ }^{58,57} \mathrm{Ni}$ and ${ }^{52} \mathrm{Fe}$, the latter two decaying to ${ }^{57} \mathrm{Fe}$ and ${ }^{52} \mathrm{Cr}$ ) and the incomplete Si-burning region $\left({ }^{58,57} \mathrm{Ni},{ }^{55} \mathrm{Co}\right.$, and ${ }^{54,52} \mathrm{Fe}$, the unstable species decaying to ${ }^{57} \mathrm{Fe},{ }^{55} \mathrm{Mn}$, and $\left.{ }^{52} \mathrm{Cr}\right)$. ${ }^{58,57} \mathrm{Ni},{ }^{55} \mathrm{Co}$, and ${ }^{54} \mathrm{Fe}$ decrease below the plot limits in the incomplete burning region while ${ }^{52} \mathrm{Fe}(\mathrm{N}=\mathrm{Z})$ increases. 

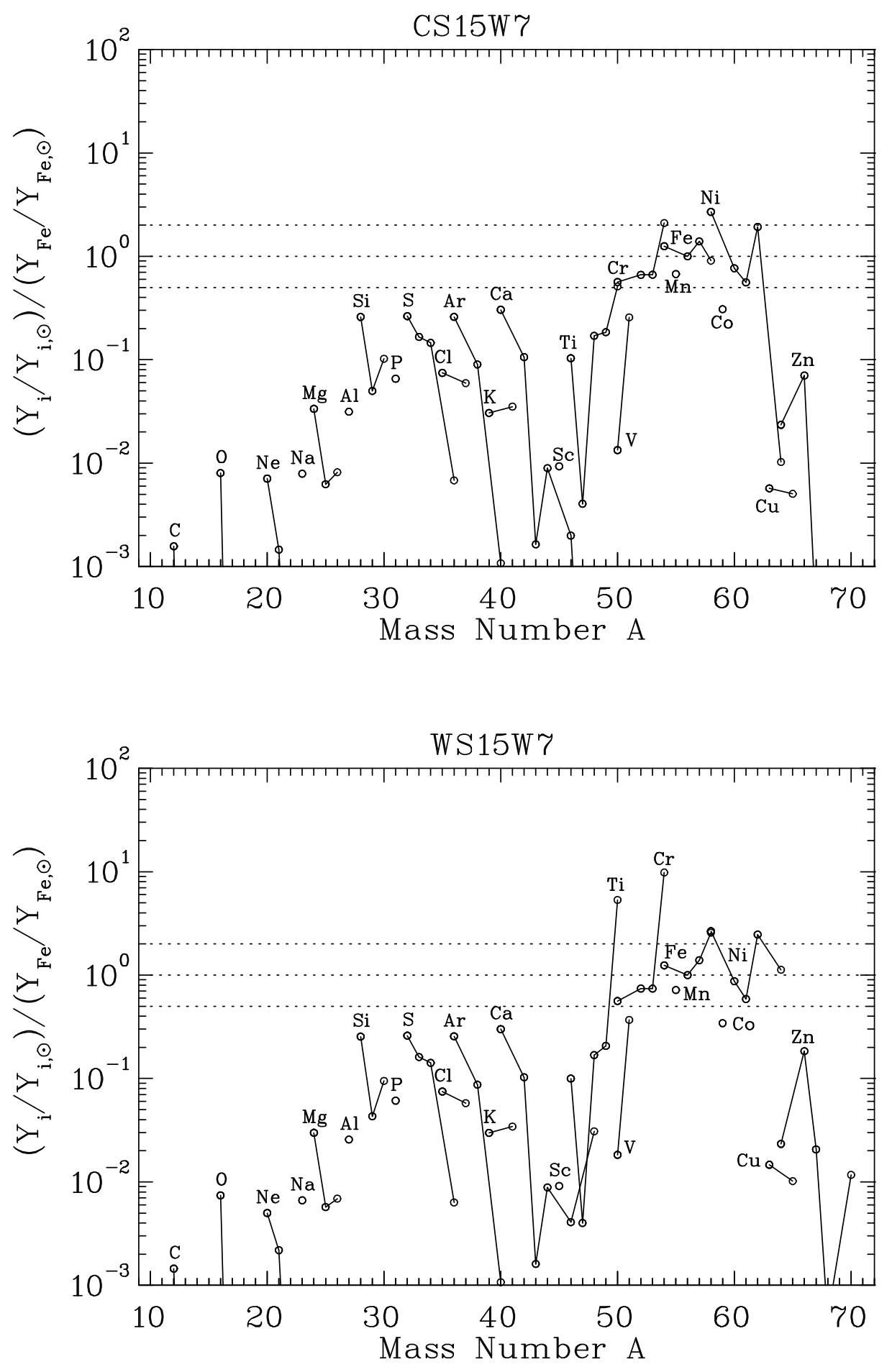

FIG. 15.- Comparison to solar for SN Ia compositions which are made up of the slow deflagrations CS15 and WS15 in the central layers and a W7 composition in the outer layers. This is mainly a test for the Fe-group composition, especially ${ }^{50} \mathrm{Ti},{ }^{54} \mathrm{Cr},{ }^{58} \mathrm{Fe}$, and ${ }^{64} \mathrm{Ni}$. 

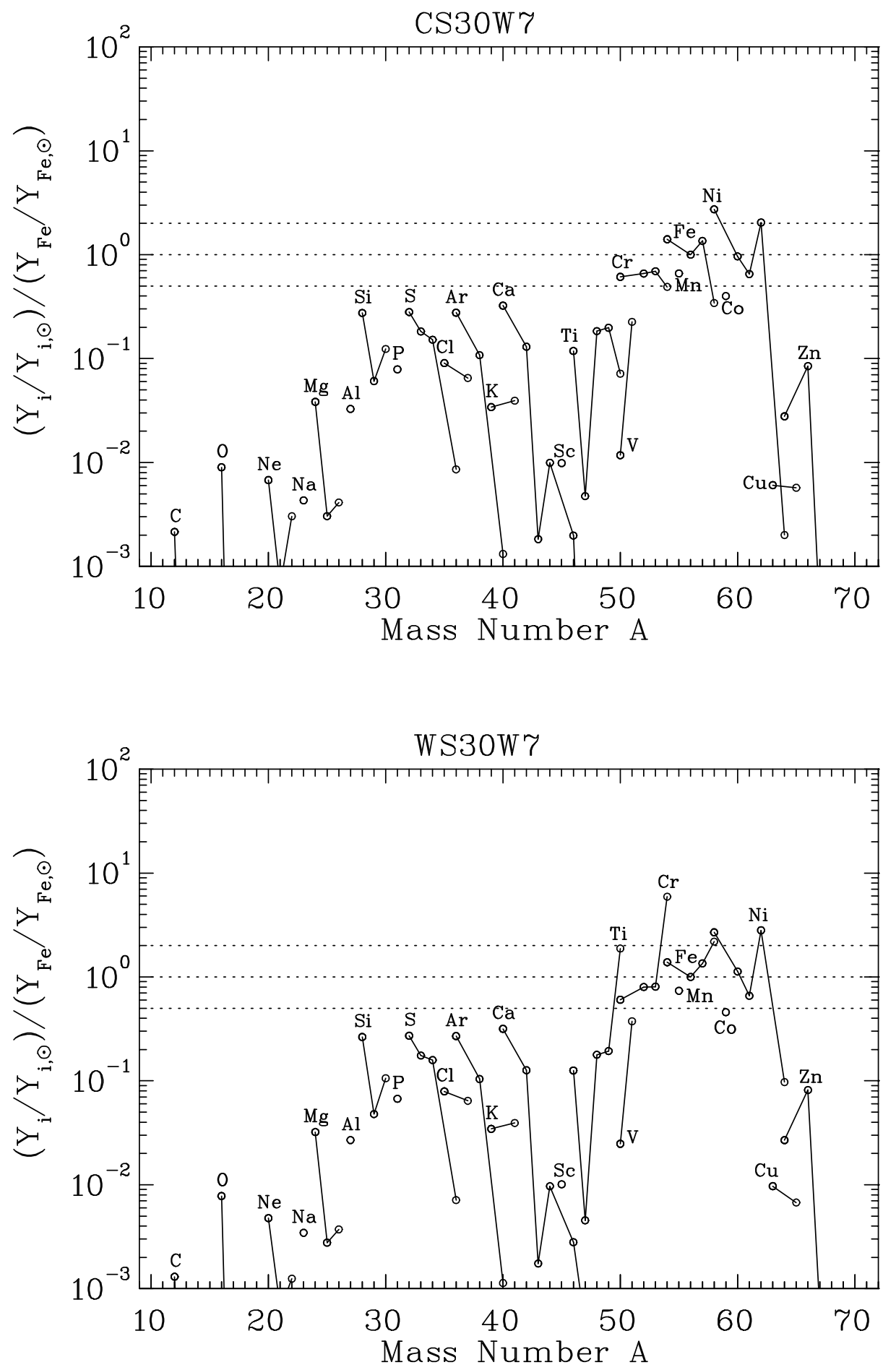

FIG. 16. - Comparison to solar for a SN Ia composition made up from CS30 and WS30 in the central layers and W7 in the outer layers. The neutron-rich species decline in comparison with CS15 and WS15. 


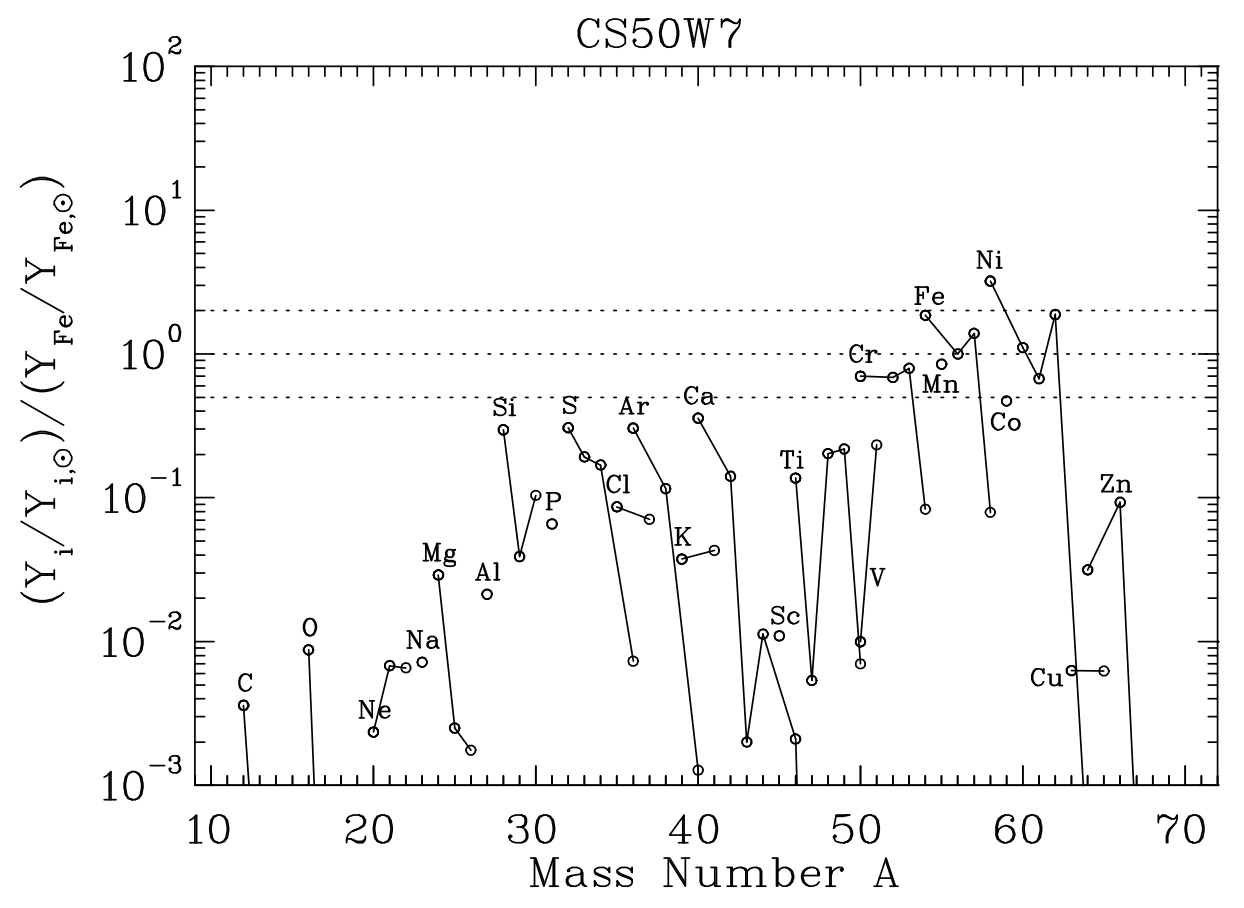

FIG. 17. - Comparison to solar for CS50 with a W7 composition in the outer layers. A further decrease of (very) neutronrich species is visible, but ${ }^{54} \mathrm{Fe}$ and ${ }^{58} \mathrm{Ni}$, originating from intermediate $Y_{e}$ regions, increase to excessive values due to the flatter $Y_{e}$-gradient of faster deflagrations (see Figure 5). 


\section{WSLW7}

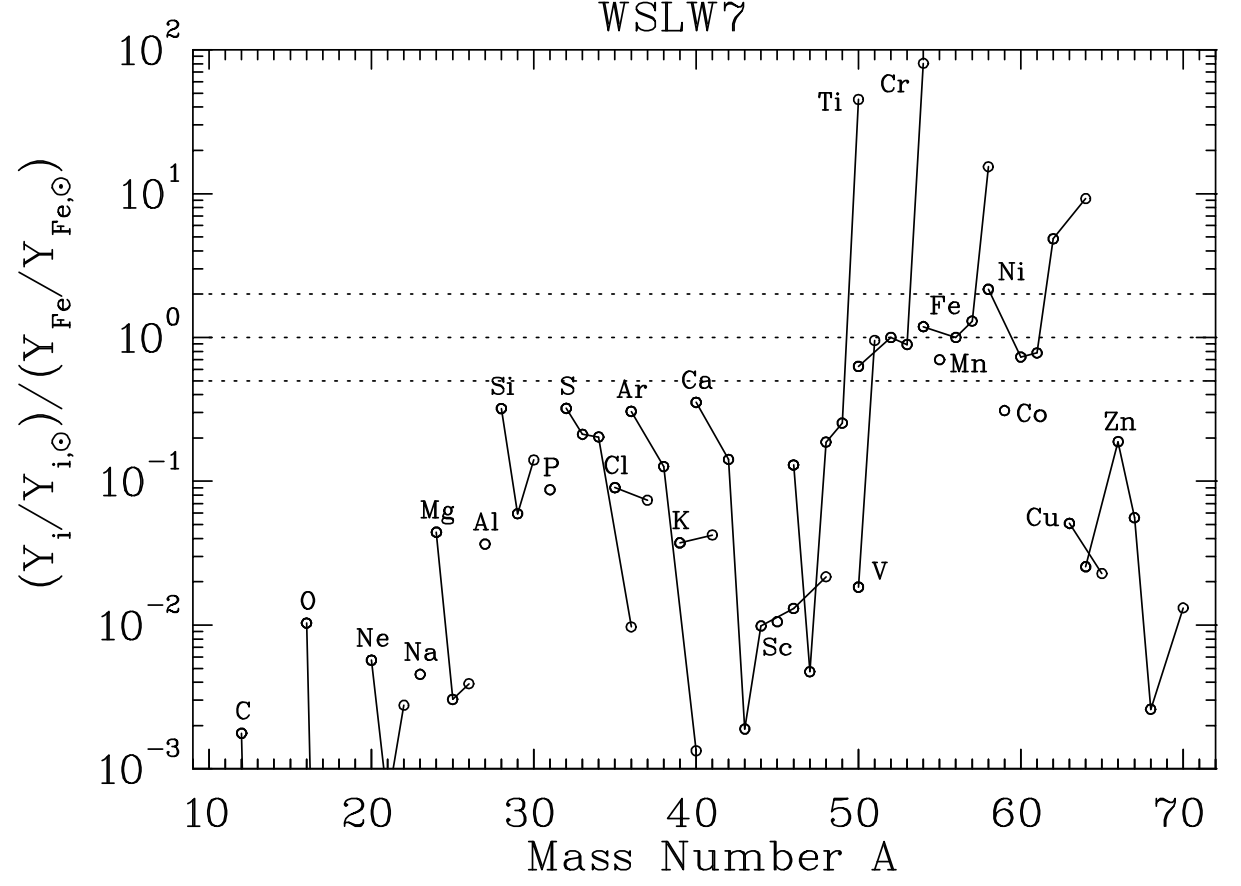

\section{WLAMWr}

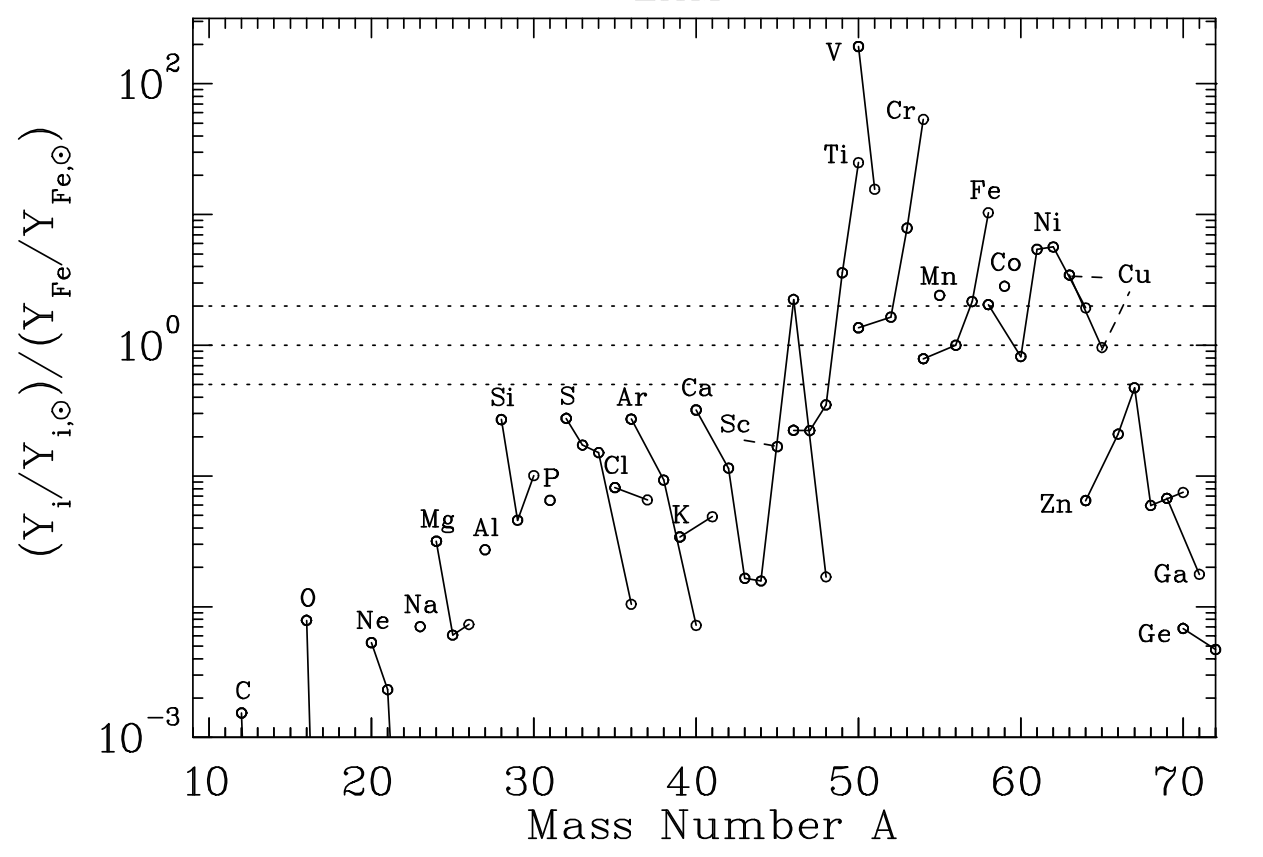

FIG. 18.- Comparison to solar for WSL and WLAM with a W7 composition in the outer layers. Excessive ratios for neutron-rich species are observed due to extended low $Y_{e}$-regions out to $0.06 \mathrm{M}_{\odot}$ (see Figure 5 ). 

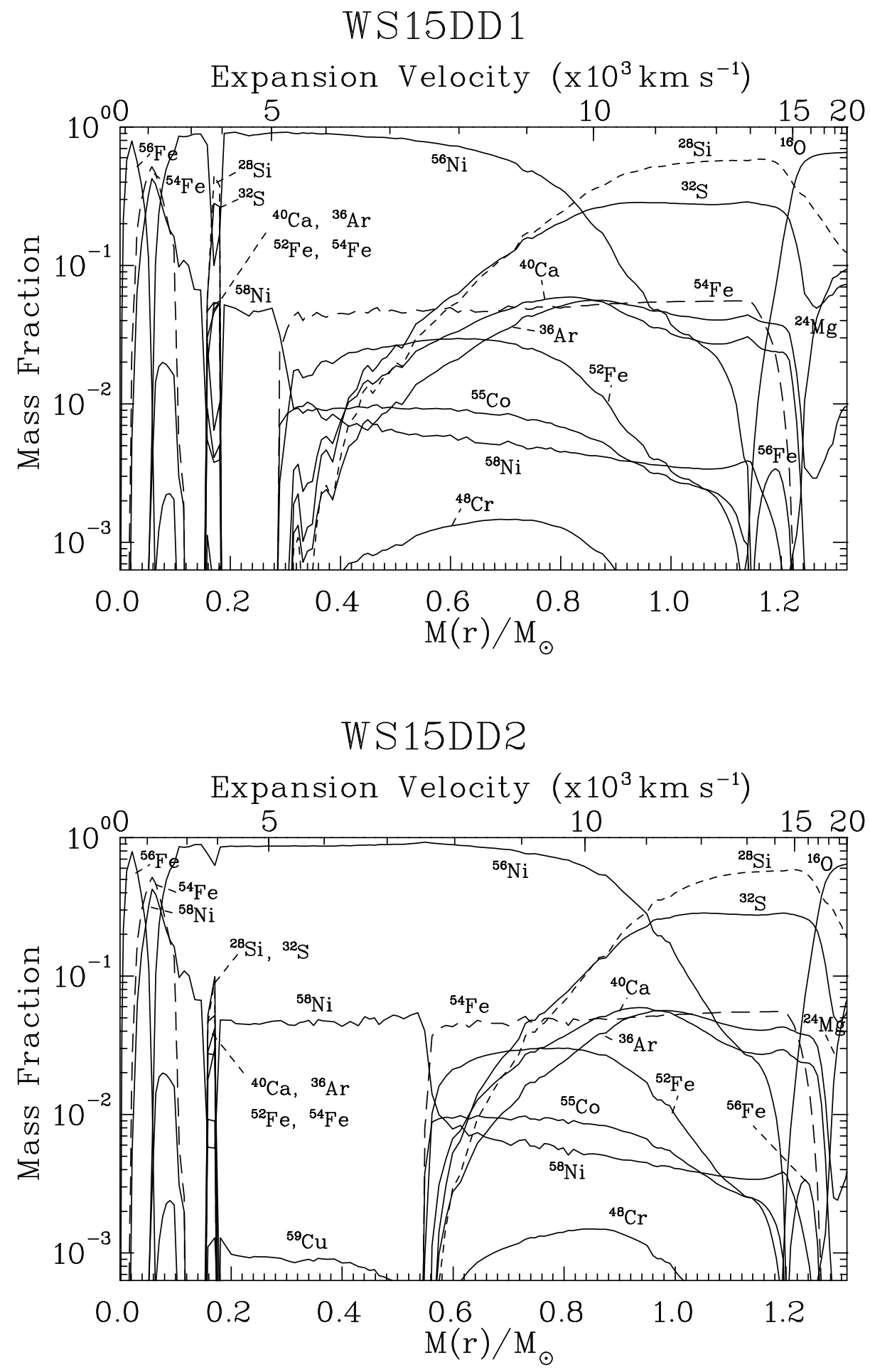

FIG. 19.- Composition of WS15DD1 and WS15DD2 against the expansion velocity and $M(r)$. 


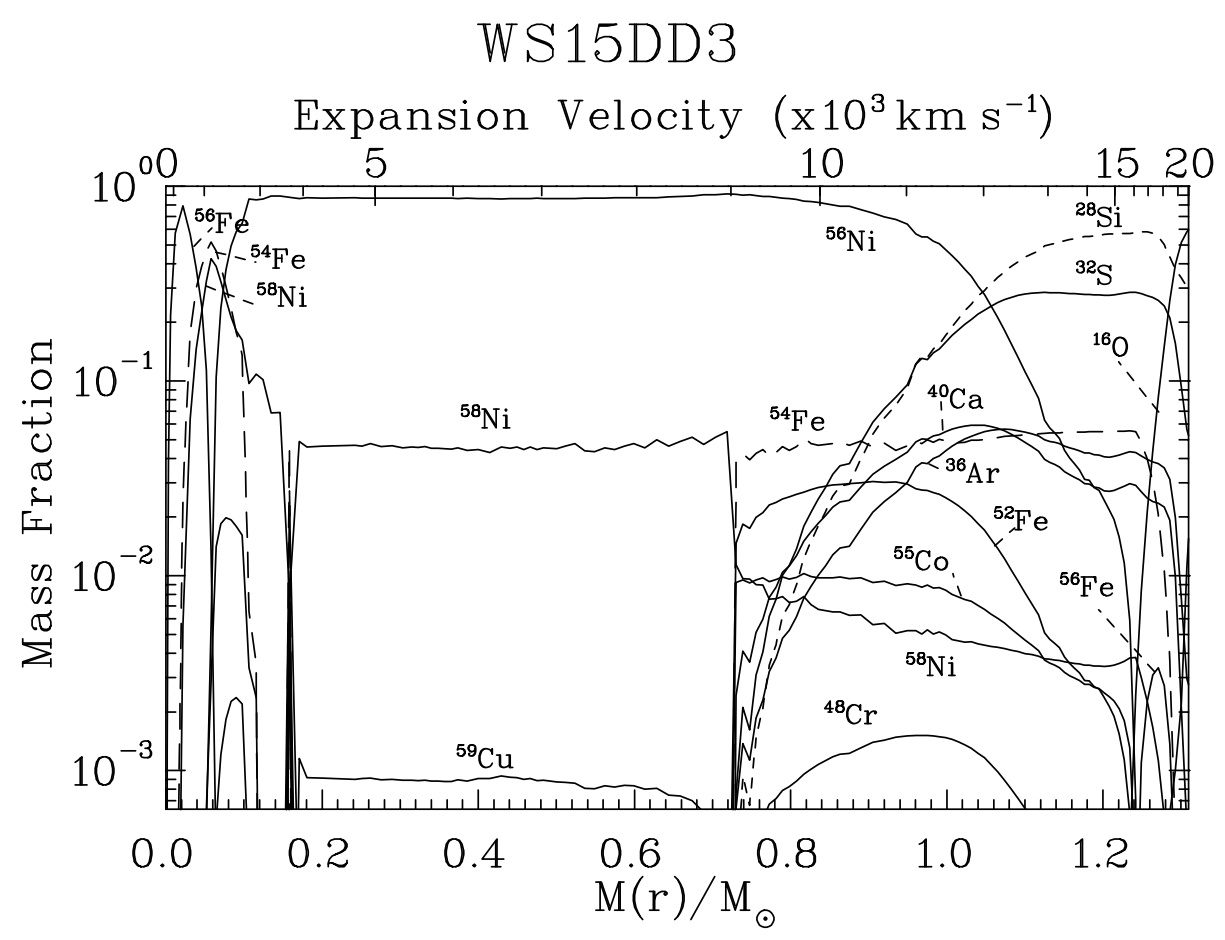

FIG. 20.- Composition of WS15DD3. In the series DD1-DD3 we see a decrease in the total amount of intermediate mass elements $(\mathrm{Si}-\mathrm{Ca})$, an increase in ${ }^{56} \mathrm{Ni}$, and a change of the ratio between matter experiencing an alpha-rich freeze-out (indicated by the ${ }^{58} \mathrm{Ni}$-plateau) and incomplete Si-burning $\left({ }^{54} \mathrm{Fe}-\right.$ plateau). 

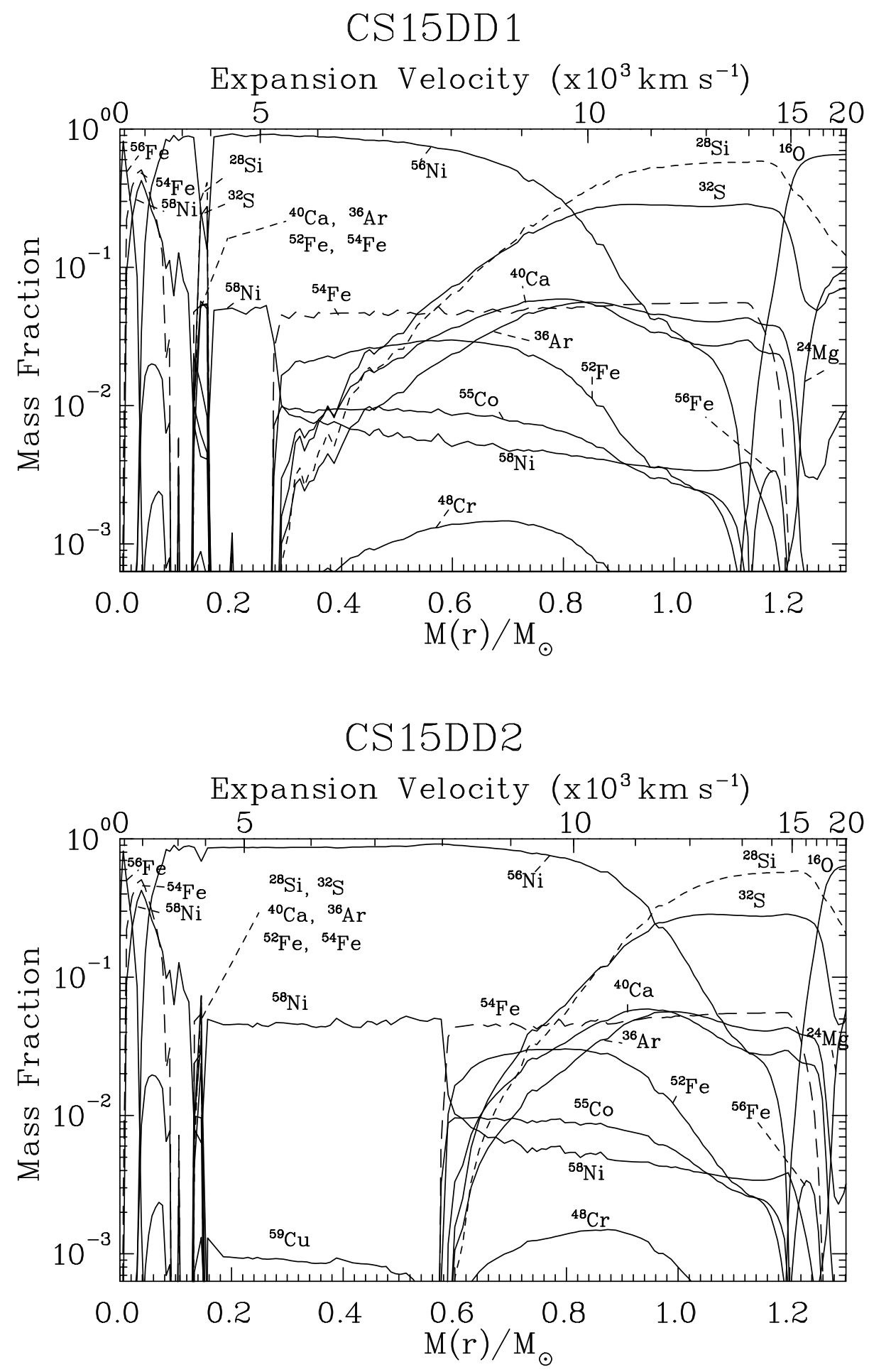

Fig. 21.- Composition of CS15DD1 and CS15DD2. The outer layers affected by the detonation behave almost identical to the WSDD series. 

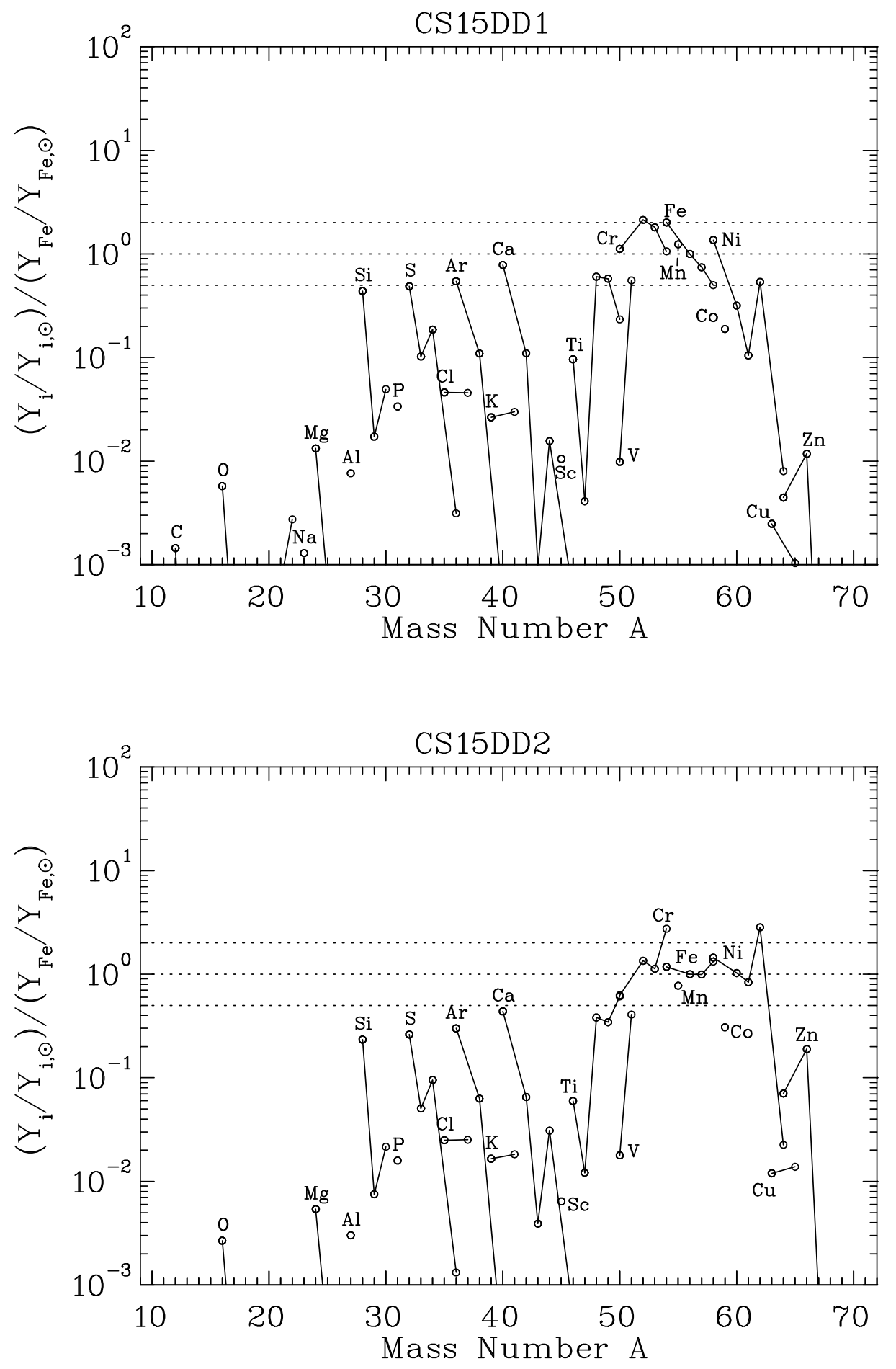

FIG. 22.- Comparison to solar for CS15DD1 and CS15DD2. A decrease of the Si-Ca/Fe ratio is observable, a decrease of e.g. ${ }^{55} \mathrm{Mn}$ and ${ }^{52} \mathrm{Cr}$ (dominated by incomplete Si-burning), and an increase of ${ }^{62} \mathrm{Ni}$ (decaying from ${ }^{62} \mathrm{Zn}$ ) and ${ }^{59} \mathrm{Co}$ (alpha-rich freeze-out). 

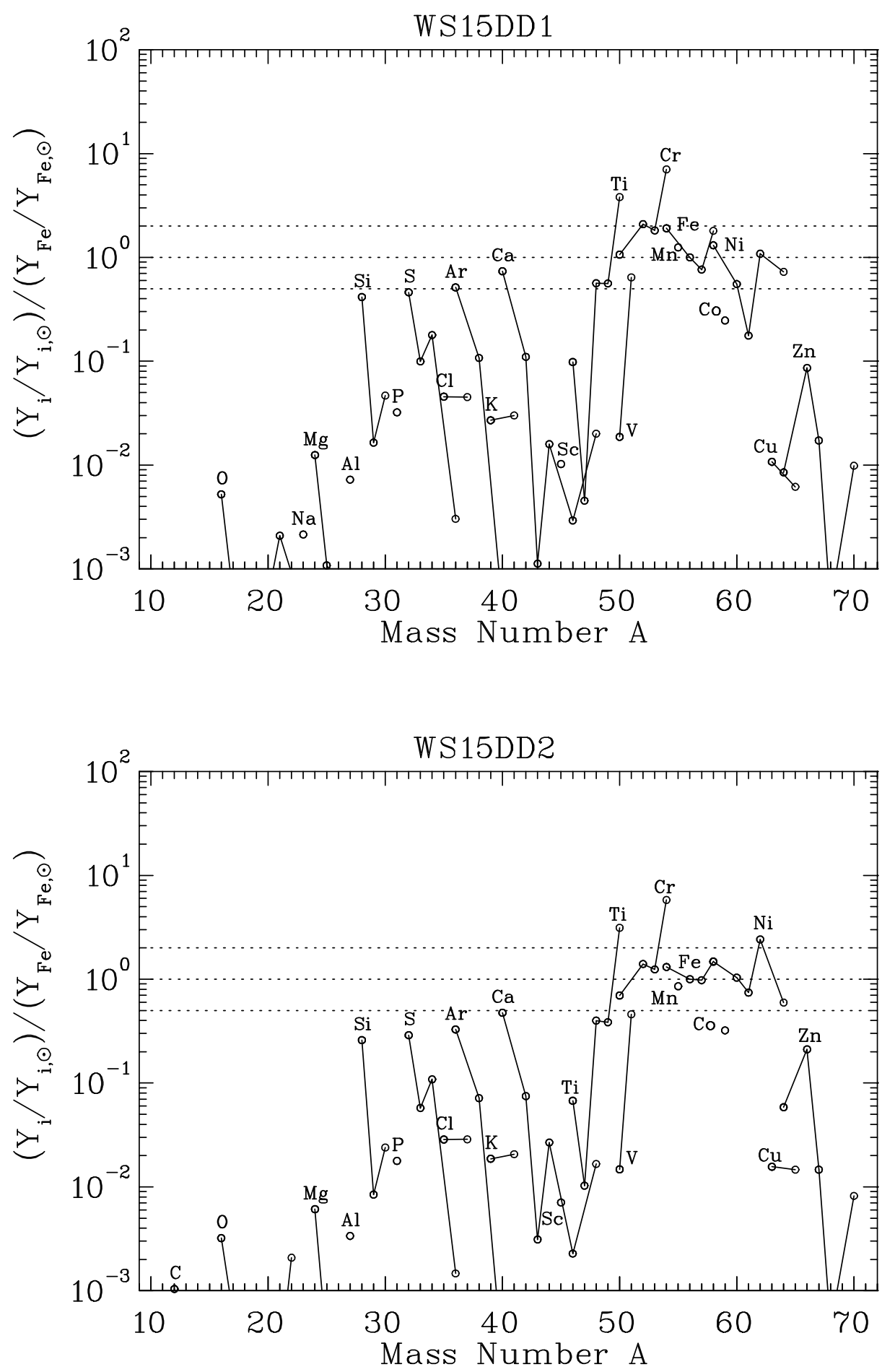

FIG. 23.- Comparison to solar for WS15DD1 and WS15DD2. 


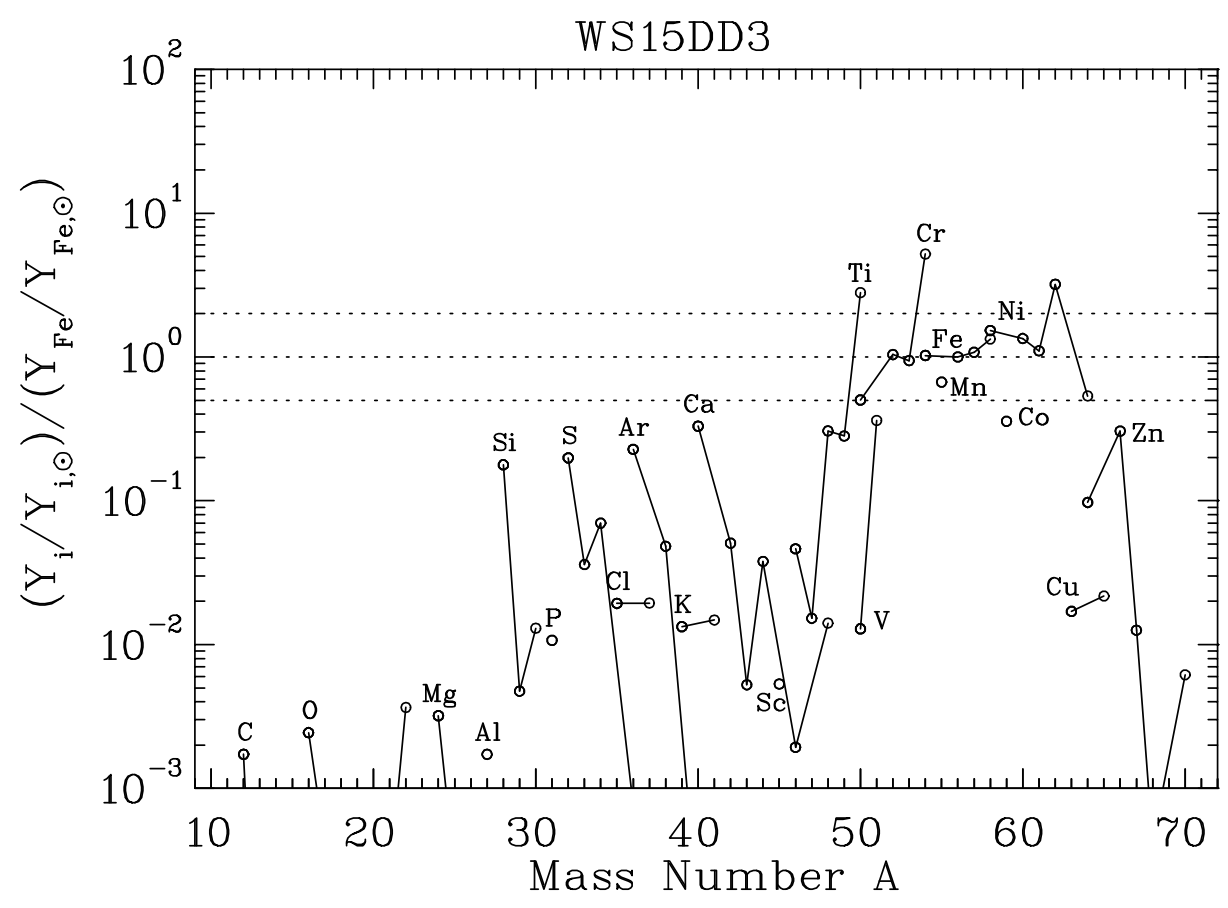

FIg. 24.- Comparison to solar for WS15DD3. In this series DD1-DD3 we see similar changes as shown in Figure 22. Differences in the neutron-rich species ${ }^{50} \mathrm{Ti},{ }^{54} \mathrm{Cr},{ }^{58} \mathrm{Fe}$, and ${ }^{62} \mathrm{Ni}$ are due to the differences between the $\mathrm{W}$ and $\mathrm{C}$ series in the central slow deflagration layers (higher ignition densities). 

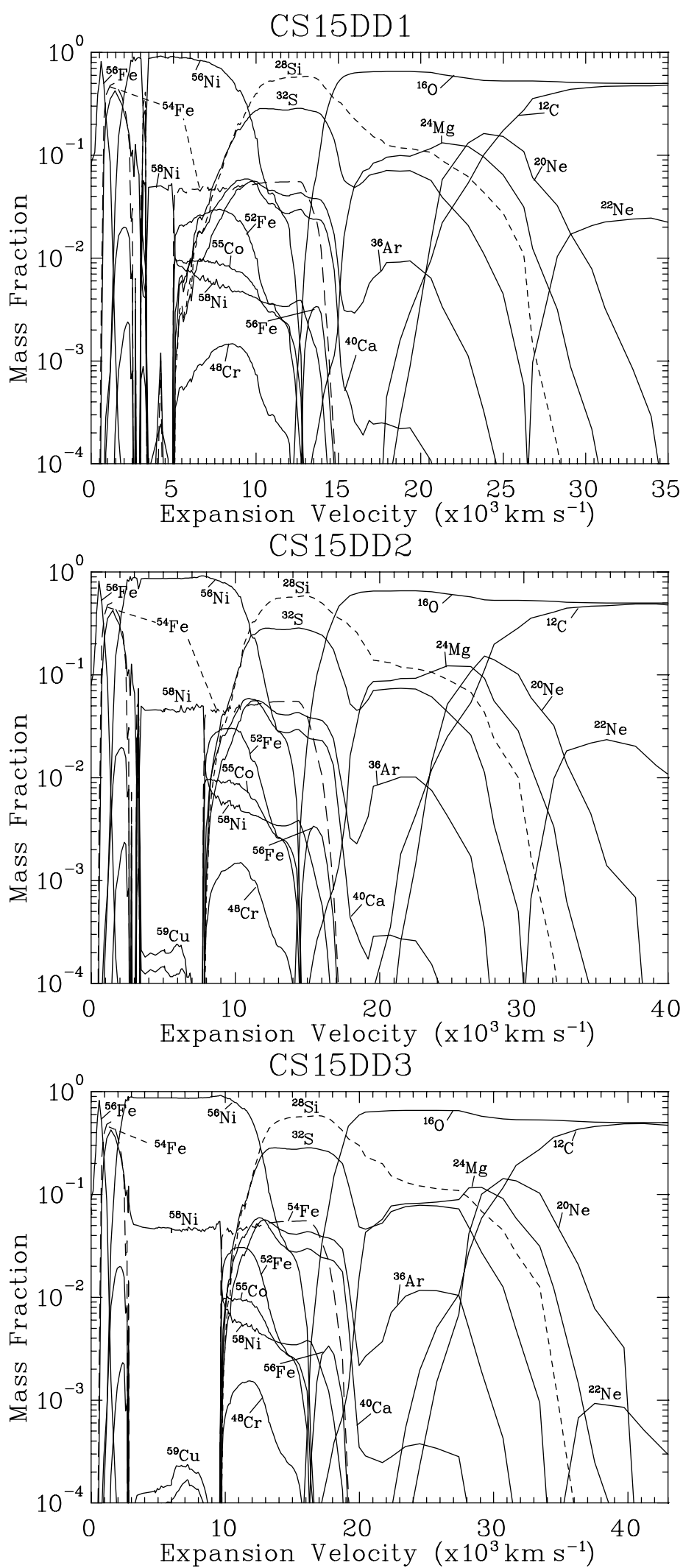

FIG. 25.- Mass fractions in the models CS15DD1-DD3 as a function of expansion velocity, which reveals more easily the outer layers affected by the deflagration-detonation transition and the quenching of nuclear burning up to unburned matter in the very outer layers. Intermediate mass elements beyond $\mathrm{Mg}$ are essentially unburned for velocities larger than 20000-30000 $\mathrm{km} \mathrm{s}^{-1}$ and would be found in their solar values scaled with the appropriate metallcity. The initial composition of the white dwarf for the present calculation consists only of ${ }^{12} \mathrm{C},{ }^{16} \mathrm{O}$, and ${ }^{22} \mathrm{Ne}$ (according to the $\mathrm{CNO}$ metallicity of the accreted matter). 
Table 1: Masses of ${ }^{56} \mathrm{Ni}$ in SN Ia Models Investigated

\begin{tabular}{crrr}
\hline Model & Mass & Energy & ${ }^{56} \mathrm{Ni} \mathrm{Mass}$ \\
\hline W7 & $1.38 \mathrm{M}_{\odot}$ & $1.3 \times 10^{51} \mathrm{ergs}$ & $0.59 \mathrm{M}_{\odot}$ \\
W70 & $1.38 \mathrm{M}_{\odot}$ & $1.3 \times 10^{51} \mathrm{ergs}$ & $0.64 \mathrm{M}_{\odot}$ \\
WS15DD1 & $1.38 \mathrm{M}_{\odot}$ & $1.33 \times 10^{51} \mathrm{ergs}$ & $0.56 \mathrm{M}_{\odot}$ \\
WS15DD2 & $1.38 \mathrm{M}_{\odot}$ & $1.40 \times 10^{51} \mathrm{ergs}$ & $0.69 \mathrm{M}_{\odot}$ \\
WS15DD3 & $1.38 \mathrm{M}_{\odot}$ & $1.43 \times 10^{51} \mathrm{ergs}$ & $0.77 \mathrm{M}_{\odot}$ \\
CS15DD1 & $1.38 \mathrm{M}_{\odot}$ & $1.34 \times 10^{51} \mathrm{ergs}$ & $0.56 \mathrm{M}_{\odot}$ \\
CS15DD2 & $1.38 \mathrm{M}_{\odot}$ & $1.44 \times 10^{51} \mathrm{ergs}$ & $0.74 \mathrm{M}_{\odot}$ \\
\hline
\end{tabular}

Table 2: Nucleosynthesis of Neutron-rich Species $\left(X_{i} / X\left({ }^{56} \mathrm{Fe}\right) /\right.$ Solar Ratio $)$

\begin{tabular}{crrrrrrrrrr}
\hline Model & $\rho_{9}$ & $v_{\text {def }} / v_{\mathrm{s}}$ & $Y_{\mathrm{e}, \mathrm{c}}$ & ${ }^{50} \mathrm{Ti}$ & ${ }^{54} \mathrm{Cr}$ & ${ }^{58} \mathrm{Fe}$ & ${ }^{64} \mathrm{Ni}$ & ${ }^{62} \mathrm{Ni}$ & ${ }^{54} \mathrm{Fe}$ & ${ }^{58} \mathrm{Ni}$ \\
\hline WSL & 2.12 & $\sim 0.01$ & 0.442 & 39 & 74 & 15 & 7 & 5 & 1.2 & 2.1 \\
WLAM & 2.12 & $\sim 0.01$ & 0.447 & 22 & 47 & 9 & 1.6 & 5 & 1.8 & 3.8 \\
WS15W7 & 2.12 & 0.015 & 0.440 & 4 & 7 & 1.7 & 0.7 & 2.1 & 1.3 & 2.7 \\
WS15DD1 & 2.12 & 0.015 & 0.440 & 4 & 7 & 1.8 & 0.7 & 1.1 & 1.9 & 1.3 \\
WS15DD2 & 2.12 & 0.015 & 0.440 & 3.1 & 6 & 1.5 & 0.6 & 2.4 & 1.3 & 1.5 \\
WS15DD3 & 2.12 & 0.015 & 0.440 & 2.8 & 5 & 1.3 & 0.5 & 3.2 & 1.0 & 1.5 \\
WS30W7 & 2.12 & 0.03 & 0.445 & 1.3 & 4 & 1.5 & 0.1 & 2.5 & 1.3 & 2.6 \\
CS15W7 & 1.37 & 0.015 & 0.449 & 0.5 & 2 & 0.9 & 0.01 & 1.9 & 1.3 & 2.7 \\
CS15DD1 & 1.37 & 0.015 & 0.449 & 0.2 & 1.1 & 0.5 & 0.01 & 0.5 & 2.0 & 1.4 \\
CS15DD2 & 1.37 & 0.015 & 0.449 & 0.6 & 2.7 & 1.3 & 0.01 & 2.8 & 1.2 & 1.4 \\
CS30W7 & 1.37 & 0.03 & 0.455 & 0.07 & 0.5 & 0.3 & 0.002 & 2.0 & 1.4 & 2.7 \\
CS50W7 & 1.37 & 0.05 & 0.459 & 0.003 & 0.04 & 0.04 & 0.0004 & 1.8 & 1.8 & 3.2 \\
W7 & 2.12 & up to 0.3 & 0.446 & 1.1 & 3.8 & 1.6 & 0.05 & 2.3 & 2.4 & 4.3 \\
W70 & 2.12 & up to 0.3 & 0.446 & 0.97 & 3.4 & 1.4 & 0.04 & 1.3 & 1.7 & 3.0 \\
\hline
\end{tabular}


Table 3: Nucleosynthesis products of SN II and Ia models

\begin{tabular}{|c|c|c|c|c|c|c|c|c|}
\hline \multicolumn{9}{|c|}{ Synthesized mass $\left(\mathrm{M}_{\odot}\right)$} \\
\hline Species & $\begin{array}{c}\text { Type II } \\
10-50 \mathrm{M}_{\odot}\end{array}$ & W7 & W70 & WDD1 & $\begin{array}{l}\text { Type Ia } \\
\text { WDD2 }\end{array}$ & WDD3 & CDD1 & CDD2 \\
\hline${ }^{12} \mathrm{C}$ & $7.93 \mathrm{E}-02$ & $4.83 \mathrm{E}-02$ & $5.08 \mathrm{E}-02$ & $5.42 \mathrm{E}-03$ & $8.99 \mathrm{E}-03$ & $1.66 \mathrm{E}-02$ & $9.93 \mathrm{E}-03$ & $5.08 \mathrm{E}-03$ \\
\hline${ }^{13} \mathrm{C}$ & $3.80 \mathrm{E}-09$ & $1.40 \mathrm{E}-06$ & $1.56 \mathrm{E}-09$ & $5.06 \mathrm{E}-07$ & $3.30 \mathrm{E}-07$ & $3.17 \mathrm{E}-07$ & $8.46 \mathrm{E}-07$ & $4.16 \mathrm{E}-07$ \\
\hline${ }^{14} \mathrm{~N}$ & $1.56 \mathrm{E}-03$ & $1.16 \mathrm{E}-06$ & $3.31 \mathrm{E}-08$ & $2.84 \mathrm{E}-04$ & $2.69 \mathrm{E}-04$ & $1.82 \mathrm{E}-04$ & $9.06 \mathrm{E}-05$ & $9.03 \mathrm{E}-05$ \\
\hline${ }^{15} \mathrm{~N}$ & $1.66 \mathrm{E}-08$ & $1.32 \mathrm{E}-09$ & $4.13 \mathrm{E}-07$ & $9.99 \mathrm{E}-07$ & $5.32 \mathrm{E}-07$ & $1.21 \mathrm{E}-07$ & $2.53 \mathrm{E}-07$ & $2.47 \mathrm{E}-07$ \\
\hline${ }^{16} \mathrm{O}$ & 1.80 & $1.43 \mathrm{E}-01$ & $1.33 \mathrm{E}-01$ & $8.82 \mathrm{E}-02$ & $6.58 \mathrm{E}-02$ & $5.58 \mathrm{E}-02$ & $9.34 \mathrm{E}-02$ & $5.83 \mathrm{E}-02$ \\
\hline${ }^{17} \mathrm{O}$ & $9.88 \mathrm{E}-08$ & $3.54 \mathrm{E}-08$ & $3.33 \mathrm{E}-10$ & $3.77 \mathrm{E}-06$ & $4.58 \mathrm{E}-06$ & $3.60 \mathrm{E}-06$ & $9.55 \mathrm{E}-07$ & $1.01 \mathrm{E}-06$ \\
\hline${ }^{18} \mathrm{O}$ & $4.61 \mathrm{E}-03$ & $8.25 \mathrm{E}-10$ & $2.69 \mathrm{E}-10$ & $6.88 \mathrm{E}-07$ & $6.35 \mathrm{E}-07$ & $2.39 \mathrm{E}-07$ & $2.08 \mathrm{E}-07$ & $1.92 \mathrm{E}-07$ \\
\hline${ }^{19} \mathrm{~F}$ & $1.16 \mathrm{E}-09$ & $5.67 \mathrm{E}-10$ & $1.37 \mathrm{E}-10$ & $1.70 \mathrm{E}-09$ & $4.50 \mathrm{E}-10$ & $2.30 \mathrm{E}-10$ & $5.83 \mathrm{E}-10$ & $4.24 \mathrm{E}-10$ \\
\hline${ }^{20} \mathrm{Ne}$ & $2.12 \mathrm{E}-01$ & $2.02 \mathrm{E}-03$ & $2.29 \mathrm{E}-03$ & $1.29 \mathrm{E}-03$ & $6.22 \mathrm{E}-04$ & $4.55 \mathrm{E}-04$ & $1.16 \mathrm{E}-03$ & $6.05 \mathrm{E}-04$ \\
\hline${ }^{21} \mathrm{Ne}$ & $1.08 \mathrm{E}-03$ & $8.46 \mathrm{E}-06$ & $2.81 \mathrm{E}-08$ & $1.16 \mathrm{E}-05$ & $1.39 \mathrm{E}-06$ & $1.72 \mathrm{E}-06$ & $3.63 \mathrm{E}-06$ & $1.99 \mathrm{E}-06$ \\
\hline${ }^{22} \mathrm{Ne}$ & $1.83 \mathrm{E}-02$ & $2.49 \mathrm{E}-03$ & 2.15E-08 & $1.51 \mathrm{E}-04$ & $4.21 \mathrm{E}-04$ & $8.25 \mathrm{E}-04$ & $4.41 \mathrm{E}-04$ & $2.11 \mathrm{E}-04$ \\
\hline${ }^{23} \mathrm{Na}$ & $6.51 \mathrm{E}-03$ & $6.32 \mathrm{E}-05$ & $1.41 \mathrm{E}-05$ & $8.77 \mathrm{E}-05$ & $2.61 \mathrm{E}-05$ & $3.01 \mathrm{E}-05$ & $5.10 \mathrm{E}-05$ & $3.50 \mathrm{E}-05$ \\
\hline${ }^{24} \mathrm{Mg}$ & 8.83E-02 & $8.50 \mathrm{E}-03$ & $1.58 \mathrm{E}-02$ & $7.55 \mathrm{E}-03$ & $4.47 \mathrm{E}-03$ & $2.62 \mathrm{E}-03$ & $7.72 \mathrm{E}-03$ & $4.20 \mathrm{E}-03$ \\
\hline${ }^{25} \mathrm{Mg}$ & $1.44 \mathrm{E}-02$ & $4.05 \mathrm{E}-05$ & 1.64E-07 & $8.23 \mathrm{E}-05$ & $2.66 \mathrm{E}-05$ & $2.68 \mathrm{E}-05$ & $4.85 \mathrm{E}-05$ & $3.25 \mathrm{E}-05$ \\
\hline${ }^{26} \mathrm{Mg}$ & $2.01 \mathrm{E}-02$ & $3.18 \mathrm{E}-05$ & $1.87 \mathrm{E}-07$ & $6.25 \mathrm{E}-05$ & $2.59 \mathrm{E}-05$ & $1.41 \mathrm{E}-05$ & $4.96 \mathrm{E}-05$ & $2.97 \mathrm{E}-05$ \\
\hline${ }^{27} \mathrm{Al}$ & $1.48 \mathrm{E}-02$ & $9.86 \mathrm{E}-04$ & $1.13 \mathrm{E}-04$ & $4.38 \mathrm{E}-04$ & $2.47 \mathrm{E}-04$ & $1.41 \mathrm{E}-04$ & $4.45 \mathrm{E}-04$ & $2.35 \mathrm{E}-04$ \\
\hline${ }^{28} \mathrm{Si}$ & $1.05 \mathrm{E}-01$ & $1.54 \mathrm{E}-01$ & $1.42 \mathrm{E}-01$ & $2.72 \mathrm{E}-01$ & $2.06 \mathrm{E}-01$ & $1.58 \mathrm{E}-01$ & $2.77 \mathrm{E}-01$ & $1.98 \mathrm{E}-01$ \\
\hline${ }^{29} \mathrm{Si}$ & $8.99 \mathrm{E}-03$ & $9.08 \mathrm{E}-04$ & $5.79 \mathrm{E}-05$ & $5.47 \mathrm{E}-04$ & $3.40 \mathrm{E}-04$ & $2.13 \mathrm{E}-04$ & $5.52 \mathrm{E}-04$ & $3.22 \mathrm{E}-04$ \\
\hline${ }^{30} \mathrm{Si}$ & $8.05 \mathrm{E}-03$ & $1.69 \mathrm{E}-03$ & $7.12 \mathrm{E}-05$ & $1.03 \mathrm{E}-03$ & $6.41 \mathrm{E}-04$ & $3.88 \mathrm{E}-04$ & $1.05 \mathrm{E}-03$ & $6.14 \mathrm{E}-04$ \\
\hline${ }^{31} \mathrm{P}$ & $1.21 \mathrm{E}-03$ & $3.57 \mathrm{E}-04$ & $9.12 \mathrm{E}-05$ & $2.38 \mathrm{E}-04$ & $1.60 \mathrm{E}-04$ & $1.07 \mathrm{E}-04$ & $2.40 \mathrm{E}-04$ & $1.52 \mathrm{E}-04$ \\
\hline${ }^{32} \mathrm{~S}$ & $3.84 \mathrm{E}-02$ & $8.46 \mathrm{E}-02$ & $9.14 \mathrm{E}-02$ & $1.60 \mathrm{E}-01$ & $1.22 \mathrm{E}-01$ & 9.37E-02 & $1.63 \mathrm{E}-01$ & $1.17 \mathrm{E}-01$ \\
\hline${ }^{33} \mathrm{~S}$ & $1.78 \mathrm{E}-04$ & $4.24 \mathrm{E}-04$ & $6.07 \mathrm{E}-05$ & $2.74 \mathrm{H}$ & $1.92 \mathrm{E}-04$ & -04 & 2.7 & $1.79 \mathrm{E}-04$ \\
\hline${ }^{34} \mathrm{~S}$ & $2.62 \mathrm{E}-03$ & $1.98 \mathrm{E}-03$ & $1.74 \mathrm{E}-05$ & $2.76 \mathrm{E}-03$ & $2.04 \mathrm{E}-03$ & $1.46 \mathrm{E}-03$ & $2.77 \mathrm{E}-03$ & $1.90 \mathrm{E}-03$ \\
\hline${ }^{36} \mathrm{~S}$ & $1.78 \mathrm{E}-06$ & $4.18 \mathrm{E}-07$ & $3.41 \mathrm{E}-11$ & $2.23 \mathrm{E}-07$ & $1.31 \mathrm{E}-07$ & $7.44 \mathrm{E}-08$ & $2.22 \mathrm{E}-07$ & $1.25 \mathrm{E}-07$ \\
\hline${ }^{35} \mathrm{Cl}$ & $1.01 \mathrm{E}-04$ & $1.37 \mathrm{E}-04$ & $1.06 \mathrm{E}-05$ & $9.28 \mathrm{E}-05$ & $7.07 \mathrm{E}-05$ & $5.33 \mathrm{E}-05$ & $9.03 \mathrm{E}-05$ & $6.56 \mathrm{E}-05$ \\
\hline${ }^{37} \mathrm{Cl}$ & $1.88 \mathrm{E}-05$ & $3.67 \mathrm{E}-05$ & $5.56 \mathrm{E}-06$ & $2.94 \mathrm{E}-05$ & $2.26 \mathrm{E}-05$ & $1.71 \mathrm{E}-05$ & $2.86 \mathrm{E}-05$ & $2.11 \mathrm{E}-05$ \\
\hline${ }^{36} \mathrm{Ar}$ & $6.62 \mathrm{E}-03$ & $1.47 \mathrm{E}-02$ & $1.91 \mathrm{E}-02$ & $3.11 \mathrm{E}-02$ & $2.41 \mathrm{E}-02$ & $1.87 \mathrm{E}-02$ & $3.18 \mathrm{E}-02$ & $2.34 \mathrm{E}-02$ \\
\hline${ }^{38} \mathrm{Ar}$ & $1.37 \mathrm{E}-03$ & $9.50 \mathrm{E}-04$ & $6.60 \mathrm{E}-07$ & $1.23 \mathrm{E}-03$ & $9.90 \mathrm{E}-04$ & $7.44 \mathrm{E}-04$ & $1.20 \mathrm{E}-03$ & $9.26 \mathrm{E}-04$ \\
\hline${ }^{40} \mathrm{Ar}$ & $2.27 \mathrm{E}-08$ & $1.87 \mathrm{E}-08$ & $3.42 \mathrm{E}-12$ & $7.81 \mathrm{E}-09$ & $5.19 \mathrm{E}-09$ & $3.56 \mathrm{E}-09$ & $7.56 \mathrm{E}-09$ & $4.82 \mathrm{E}-09$ \\
\hline
\end{tabular}


Continued form TABLE 3 .

\begin{tabular}{|c|c|c|c|c|c|c|c|c|}
\hline \multicolumn{9}{|c|}{ Synthesized mass $\left(\mathrm{M}_{\odot}\right)$} \\
\hline Species & $\begin{array}{c}\text { Type II } \\
10-50 \mathrm{M}_{\odot}\end{array}$ & W7 & W70 & WDD1 & $\begin{array}{l}\text { Type la } \\
\text { WDD2 }\end{array}$ & WDD3 & CDD1 & CDD2 \\
\hline${ }^{39} \mathrm{~K}$ & $6.23 \mathrm{E}-05$ & $7.23 \mathrm{E}-05$ & $1.67 \mathrm{E}-06$ & $6.76 \mathrm{E}-05$ & $5.67 \mathrm{E}-05$ & $4.52 \mathrm{E}-05$ & $6.39 \mathrm{E}-05$ & $5.34 \mathrm{E}-05$ \\
\hline${ }^{41} \mathrm{~K}$ & $5.07 \mathrm{E}-06$ & $6.11 \mathrm{E}-06$ & 4.83E-07 & 5.43E-06 & $4.52 \mathrm{E}-06$ & $3.62 \mathrm{E}-06$ & $5.20 \mathrm{E}-06$ & $4.25 \mathrm{E}-06$ \\
\hline${ }^{40} \mathrm{Ca}$ & $5.77 \mathrm{E}-03$ & $1.19 \mathrm{E}-02$ & $1.81 \mathrm{E}-02$ & $3.10 \mathrm{E}-02$ & $2.43 \mathrm{E}-02$ & $1.88 \mathrm{E}-02$ & $3.18 \mathrm{E}-02$ & $2.38 \mathrm{E}-02$ \\
\hline${ }^{42} \mathrm{Ca}$ & $4.23 \mathrm{E}-05$ & $2.82 \mathrm{E}-05$ & $1.06 \mathrm{E}-08$ & $3.09 \mathrm{E}-05$ & $2.55 \mathrm{E}-05$ & $1.93 \mathrm{E}-05$ & $2.97 \mathrm{E}-05$ & $2.36 \mathrm{E}-05$ \\
\hline${ }^{43} \mathrm{Ca}$ & $1.08 \mathrm{E}-06$ & $9.64 \mathrm{E}-08$ & $6.17 \mathrm{E}-08$ & $6.60 \mathrm{E}-08$ & $2.22 \mathrm{E}-07$ & $4.18 \mathrm{E}-07$ & $5.15 \mathrm{E}-08$ & $2.96 \mathrm{E}-07$ \\
\hline${ }^{44} \mathrm{Ca}$ & $5.53 \mathrm{E}-05$ & 8.02E-06 & $1.38 \mathrm{E}-05$ & $1.44 \mathrm{E}-05$ & $2.95 \mathrm{E}-05$ & $4.66 \mathrm{E}-05$ & $1.37 \mathrm{E}-05$ & $3.62 \mathrm{E}-05$ \\
\hline${ }^{46} \mathrm{Ca}$ & $1.43 \mathrm{E}-10$ & $4.16 \mathrm{E}-09$ & 1.01E-09 & $5.01 \mathrm{E}-09$ & $4.73 \mathrm{E}-09$ & $4.47 \mathrm{E}-09$ & $8.79 \mathrm{E}-10$ & $1.18 \mathrm{E}-09$ \\
\hline${ }^{48} \mathrm{Ca}$ & $5.33 \mathrm{E}-14$ & $2.60 \mathrm{E}-09$ & $2.47 \mathrm{E}-09$ & $1.63 \mathrm{E}-06$ & $1.64 \mathrm{E}-06$ & $1.55 \mathrm{E}-06$ & $3.54 \mathrm{E}-11$ & $4.93 \mathrm{E}-10$ \\
\hline${ }^{45} \mathrm{Sc}$ & $2.29 \mathrm{E}-07$ & $2.21 \mathrm{E}-07$ & $3.85 \mathrm{E}-08$ & $2.49 \mathrm{E}-07$ & $2.09 \mathrm{E}-07$ & $1.76 \mathrm{E}-07$ & $2.47 \mathrm{E}-07$ & $2.02 \mathrm{E}-07$ \\
\hline${ }^{46} \mathrm{Ti}$ & $7.48 \mathrm{E}-06$ & $1.33 \mathrm{E}-05$ & $3.49 \mathrm{E}-07$ & $1.34 \mathrm{E}-05$ & $1.12 \mathrm{E}-05$ & $8.58 \mathrm{E}-06$ & $1.27 \mathrm{E}-05$ & $1.05 \mathrm{E}-05$ \\
\hline${ }^{47} \mathrm{Ti}$ & $2.11 \mathrm{E}-06$ & $5.10 \mathrm{E}-07$ & $4.08 \mathrm{E}-07$ & $5.65 \mathrm{E}-07$ & $1.56 \mathrm{E}-06$ & $2.57 \mathrm{E}-06$ & $4.93 \mathrm{E}-07$ & $1.95 \mathrm{E}-06$ \\
\hline${ }^{48} \mathrm{Ti}$ & $1.16 \mathrm{E}-04$ & $2.05 \mathrm{E}-04$ & $3.13 \mathrm{E}-04$ & $7.10 \mathrm{E}-04$ & $6.11 \mathrm{E}-04$ & $5.23 \mathrm{E}-04$ & 7.32E-04 & $6.20 \mathrm{E}-04$ \\
\hline${ }^{49} \mathrm{Ti}$ & $5.98 \mathrm{E}-06$ & $1.71 \mathrm{E}-05$ & $2.94 \mathrm{E}-06$ & $5.27 \mathrm{E}-05$ & $4.39 \mathrm{E}-05$ & $3.59 \mathrm{E}-05$ & $5.22 \mathrm{E}-05$ & $4.17 \mathrm{E}-05$ \\
\hline${ }^{50} \mathrm{Ti}$ & $3.81 \mathrm{E}-10$ & $1.07 \mathrm{E}-04$ & $1.04 \mathrm{E}-04$ & $3.52 \mathrm{E}-04$ & $3.51 \mathrm{E}-04$ & $3.51 \mathrm{E}-04$ & $2.08 \mathrm{E}-05$ & $7.28 \mathrm{E}-05$ \\
\hline${ }^{50} \mathrm{~V}$ & $7.25 \mathrm{E}-10$ & $1.55 \mathrm{E}-08$ & $1.22 \mathrm{E}-08$ & $9.74 \mathrm{E}-09$ & $9.33 \mathrm{E}-09$ & $9.07 \mathrm{E}-09$ & $4.94 \mathrm{E}-09$ & $1.20 \mathrm{E}-08$ \\
\hline${ }^{51} \mathrm{~V}$ & $1.00 \mathrm{E}-05$ & $7.49 \mathrm{E}-05$ & $4.27 \mathrm{E}-05$ & $1.33 \mathrm{E}-04$ & $1.16 \mathrm{E}-04$ & $1.02 \mathrm{E}-04$ & $1.11 \mathrm{E}-04$ & $1.09 \mathrm{E}-04$ \\
\hline${ }^{50} \mathrm{Cr}$ & $4.64 \mathrm{E}-05$ & $2.73 \mathrm{E}-04$ & $6.65 \mathrm{E}-05$ & $4.44 \mathrm{E}-04$ & $3.53 \mathrm{E}-04$ & $2.84 \mathrm{E}-04$ & $4.49 \mathrm{E}-04$ & $3.36 \mathrm{E}-04$ \\
\hline${ }^{52} \mathrm{Cr}$ & $1.15 \mathrm{E}-03$ & $6.36 \mathrm{E}-03$ & 7.73E-03 & $1.68 \mathrm{E}-02$ & $1.37 \mathrm{E}-02$ & $1.13 \mathrm{E}-02$ & $1.65 \mathrm{E}-02$ & $1.40 \mathrm{E}-02$ \\
\hline${ }^{53} \mathrm{Cr}$ & 1.19E-04 & $9.22 \mathrm{E}-04$ & $5.66 \mathrm{E}-04$ & 1.66E-03 & $1.38 \mathrm{E}-03$ & $1.17 \mathrm{E}-03$ & $1.59 \mathrm{E}-03$ & $1.33 \mathrm{E}-03$ \\
\hline${ }^{54} \mathrm{Cr}$ & $2.33 \mathrm{E}-08$ & $9.24 \mathrm{E}-04$ & $9.04 \mathrm{E}-04$ & $1.60 \mathrm{E}-03$ & $1.60 \mathrm{E}-03$ & $1.60 \mathrm{E}-03$ & $2.31 \mathrm{E}-04$ & $8.02 \mathrm{E}-04$ \\
\hline${ }^{55} \mathrm{Mn}$ & $3.86 \mathrm{E}-04$ & 8.87E-03 & $6.66 \mathrm{E}-03$ & $8.48 \mathrm{E}-03$ & $7.05 \mathrm{E}-03$ & $6.16 \mathrm{E}-03$ & $8.10 \mathrm{E}-03$ & $6.77 \mathrm{E}-03$ \\
\hline${ }^{54} \mathrm{Fe}$ & $3.62 \mathrm{E}-03$ & $9.55 \mathrm{E}-02$ & 7.30E-02 & $7.08 \mathrm{E}-02$ & $5.91 \mathrm{E}-02$ & $5.15 \mathrm{E}-02$ & $7.20 \mathrm{E}-02$ & $5.64 \mathrm{E}-02$ \\
\hline${ }^{56} \mathrm{Fe}$ & $8.44 \mathrm{E}-02$ & $6.26 \mathrm{E}-01$ & $6.80 \mathrm{E}-01$ & $5.87 \mathrm{E}-01$ & $7.13 \mathrm{E}-01$ & $7.95 \mathrm{E}-01$ & $5.65 \mathrm{E}-01$ & $7.57 \mathrm{E}-01$ \\
\hline${ }^{57} \mathrm{Fe}$ & $2.72 \mathrm{E}-03$ & $2.45 \mathrm{E}-02$ & $1.92 \mathrm{E}-02$ & $1.08 \mathrm{E}-02$ & $1.67 \mathrm{E}-02$ & $2.06 \mathrm{E}-02$ & $1.01 \mathrm{E}-02$ & $1.80 \mathrm{E}-02$ \\
\hline${ }^{58} \mathrm{Fe}$ & $7.22 \mathrm{E}-09$ & $3.03 \mathrm{E}-03$ & $2.96 \mathrm{E}-03$ & $3.23 \mathrm{E}-03$ & $3.23 \mathrm{E}-03$ & $3.24 \mathrm{E}-03$ & $8.63 \mathrm{E}-04$ & $3.06 \mathrm{E}-03$ \\
\hline${ }^{59} \mathrm{Co}$ & 7.27E-05 & $1.04 \mathrm{E}-03$ & $9.68 \mathrm{E}-04$ & $3.95 \mathrm{E}-04$ & $6.25 \mathrm{E}-04$ & $7.75 \mathrm{E}-04$ & $2.91 \mathrm{E}-04$ & $6.35 \mathrm{E}-04$ \\
\hline${ }^{58} \mathrm{Ni}$ & $3.63 \mathrm{E}-03$ & $1.10 \mathrm{E}-01$ & $8.34 \mathrm{E}-02$ & $3.14 \mathrm{E}-02$ & $4.29 \mathrm{E}-02$ & $4.97 \mathrm{E}-02$ & $3.15 \mathrm{E}-02$ & $4.47 \mathrm{E}-02$ \\
\hline${ }^{60} \mathrm{Ni}$ & $1.75 \mathrm{E}-03$ & $1.24 \mathrm{E}-02$ & 1.47E-02 & $5.08 \mathrm{E}-03$ & $1.15 \mathrm{E}-02$ & $1.67 \mathrm{E}-02$ & $2.81 \mathrm{E}-03$ & $1.21 \mathrm{E}-02$ \\
\hline${ }^{61} \mathrm{Ni}$ & $8.35 \mathrm{E}-05$ & $2.35 \mathrm{E}-04$ & $2.15 \mathrm{E}-04$ & $7.00 \mathrm{E}-05$ & $3.58 \mathrm{E}-04$ & $5.92 \mathrm{E}-04$ & $4.00 \mathrm{E}-05$ & $4.27 \mathrm{E}-04$ \\
\hline
\end{tabular}

Continued form TABLE 3.

\begin{tabular}{lcccccccc}
\hline \hline & \multicolumn{7}{c}{ Synthesized mass (M $\left.{ }_{\odot}\right)$} \\
\cline { 2 - 9 } & Type II & & & Type Ia & & \\
Species & $10-50 \mathrm{M}_{\odot}$ & W7 & W70 & WDD1 & WDD2 & WDD3 & CDD1 & CDD2 \\
\hline${ }^{62} \mathrm{Ni}$ & $5.09 \mathrm{E}-04$ & $3.07 \mathrm{E}-03$ & $1.85 \mathrm{E}-03$ & $1.37 \mathrm{E}-03$ & $3.69 \mathrm{E}-03$ & $5.46 \mathrm{E}-03$ & $6.51 \mathrm{E}-04$ & $4.60 \mathrm{E}-03$ \\
${ }^{64} \mathrm{Ni}$ & $3.20 \mathrm{E}-14$ & $1.70 \mathrm{E}-05$ & $1.65 \mathrm{E}-05$ & $2.32 \mathrm{E}-04$ & $2.31 \mathrm{E}-04$ & $2.32 \mathrm{E}-04$ & $2.47 \mathrm{E}-06$ & $9.29 \mathrm{E}-06$ \\
${ }^{63} \mathrm{Cu}$ & $8.37 \mathrm{E}-07$ & $2.32 \mathrm{E}-06$ & $3.00 \mathrm{E}-06$ & $2.77 \mathrm{E}-06$ & $4.88 \mathrm{E}-06$ & $5.92 \mathrm{E}-06$ & $6.14 \mathrm{E}-07$ & $3.97 \mathrm{E}-06$ \\
${ }^{65} \mathrm{Cu}$ & $4.07 \mathrm{E}-07$ & $6.84 \mathrm{E}-07$ & $8.33 \mathrm{E}-07$ & $7.08 \mathrm{E}-07$ & $2.04 \mathrm{E}-06$ & $3.38 \mathrm{E}-06$ & $1.14 \mathrm{E}-07$ & $2.05 \mathrm{E}-06$ \\
${ }^{64} \mathrm{Zn}$ & $1.03 \mathrm{E}-05$ & $1.06 \mathrm{E}-05$ & $7.01 \mathrm{E}-05$ & $3.71 \mathrm{E}-06$ & $3.10 \mathrm{E}-05$ & $5.76 \mathrm{E}-05$ & $1.87 \mathrm{E}-06$ & $3.96 \mathrm{E}-05$ \\
${ }^{66} \mathrm{Zn}$ & $8.61 \mathrm{E}-06$ & $1.76 \mathrm{E}-05$ & $6.26 \mathrm{E}-06$ & $2.16 \mathrm{E}-05$ & $6.42 \mathrm{E}-05$ & $1.04 \mathrm{E}-04$ & $2.84 \mathrm{E}-06$ & $6.11 \mathrm{E}-05$ \\
${ }^{67} \mathrm{Zn}$ & $1.52 \mathrm{E}-08$ & $1.58 \mathrm{E}-08$ & $7.28 \mathrm{E}-09$ & $6.35 \mathrm{E}-07$ & $6.55 \mathrm{E}-07$ & $6.27 \mathrm{E}-07$ & $1.69 \mathrm{E}-09$ & $4.01 \mathrm{E}-08$ \\
${ }^{68} \mathrm{Zn}$ & $3.92 \mathrm{E}-09$ & $1.74 \mathrm{E}-08$ & $1.13 \mathrm{E}-08$ & $7.44 \mathrm{E}-08$ & $8.81 \mathrm{E}-08$ & $9.42 \mathrm{E}-08$ & $3.08 \mathrm{E}-09$ & $3.03 \mathrm{E}-08$ \\
\hline
\end{tabular}


Table 4: Major Radioactive Species in SN Ia Models

\begin{tabular}{lccccccc}
\hline \hline & \multicolumn{7}{c}{ Synthesized mass $\left(\mathrm{M}_{\odot}\right)$} \\
\cline { 2 - 8 } Species & W7 & W70 & WDD1 & WDpe la & WDD3 & CDD1 & CDD2 \\
\hline${ }^{22} \mathrm{Na}$ & $1.73 \mathrm{E}-08$ & $1.08 \mathrm{E}-08$ & $2.66 \mathrm{E}-08$ & $1.33 \mathrm{E}-08$ & $3.04 \mathrm{E}-08$ & $1.68 \mathrm{E}-08$ & $9.64 \mathrm{E}-09$ \\
${ }^{26} \mathrm{Al}$ & $4.93 \mathrm{E}-07$ & $2.92 \mathrm{E}-08$ & $4.61 \mathrm{E}-07$ & $1.88 \mathrm{E}-07$ & $1.64 \mathrm{E}-07$ & $3.81 \mathrm{E}-07$ & $2.32 \mathrm{E}-07$ \\
${ }^{36} \mathrm{Cl}$ & $2.58 \mathrm{E}-06$ & $3.97 \mathrm{E}-10$ & $1.25 \mathrm{E}-06$ & $7.85 \mathrm{E}-07$ & $4.89 \mathrm{E}-07$ & $1.23 \mathrm{E}-06$ & $7.35 \mathrm{E}-07$ \\
${ }^{39} \mathrm{Ar}$ & $1.20 \mathrm{E}-08$ & $2.00 \mathrm{E}-13$ & $6.04 \mathrm{E}-09$ & $4.44 \mathrm{E}-09$ & $3.29 \mathrm{E}-09$ & $5.99 \mathrm{E}-09$ & $4.12 \mathrm{E}-09$ \\
${ }^{40} \mathrm{~K}$ & $8.44 \mathrm{E}-08$ & $5.46 \mathrm{E}-12$ & $3.29 \mathrm{E}-08$ & $2.35 \mathrm{E}-08$ & $1.73 \mathrm{E}-08$ & $3.13 \mathrm{E}-08$ & $2.15 \mathrm{E}-08$ \\
${ }^{41} \mathrm{Ca}$ & $6.09 \mathrm{E}-06$ & $4.83 \mathrm{E}-07$ & $5.42 \mathrm{E}-06$ & $4.52 \mathrm{E}-06$ & $3.62 \mathrm{E}-06$ & $5.20 \mathrm{E}-06$ & $4.25 \mathrm{E}-06$ \\
${ }^{44} \mathrm{Ti}$ & $7.94 \mathrm{E}-06$ & $1.38 \mathrm{E}-05$ & $1.44 \mathrm{E}-05$ & $2.95 \mathrm{E}-05$ & $4.65 \mathrm{E}-05$ & $1.36 \mathrm{E}-05$ & $3.62 \mathrm{E}-05$ \\
${ }^{48} \mathrm{~V}$ & $4.95 \mathrm{E}-08$ & $1.61 \mathrm{E}-08$ & $6.52 \mathrm{E}-08$ & $5.38 \mathrm{E}-08$ & $4.11 \mathrm{E}-08$ & $6.33 \mathrm{E}-08$ & $5.03 \mathrm{E}-08$ \\
${ }^{49} \mathrm{~V}$ & $1.52 \mathrm{E}-07$ & $3.23 \mathrm{E}-08$ & $1.18 \mathrm{E}-07$ & $1.03 \mathrm{E}-07$ & $8.63 \mathrm{E}-08$ & $9.99 \mathrm{E}-08$ & $8.88 \mathrm{E}-08$ \\
${ }^{53} \mathrm{Mn}$ & $2.77 \mathrm{E}-04$ & $2.48 \mathrm{E}-04$ & $1.58 \mathrm{E}-04$ & $1.54 \mathrm{E}-04$ & $1.50 \mathrm{E}-04$ & $8.47 \mathrm{E}-05$ & $9.69 \mathrm{E}-05$ \\
${ }^{60} \mathrm{Fe}$ & $7.52 \mathrm{E}-07$ & $7.19 \mathrm{E}-07$ & $7.33 \mathrm{E}-05$ & $7.33 \mathrm{E}-05$ & $7.36 \mathrm{E}-05$ & $6.23 \mathrm{E}-08$ & $2.74 \mathrm{E}-07$ \\
${ }^{56} \mathrm{Co}$ & $1.44 \mathrm{E}-04$ & $1.26 \mathrm{E}-04$ & $6.04 \mathrm{E}-05$ & $5.51 \mathrm{E}-05$ & $5.20 \mathrm{E}-05$ & $6.18 \mathrm{E}-05$ & $5.24 \mathrm{E}-05$ \\
${ }^{57} \mathrm{Co}$ & $1.48 \mathrm{E}-03$ & $1.42 \mathrm{E}-03$ & $6.43 \mathrm{E}-04$ & $6.42 \mathrm{E}-04$ & $6.36 \mathrm{E}-04$ & $4.50 \mathrm{E}-04$ & $4.47 \mathrm{E}-04$ \\
${ }^{60} \mathrm{Co}$ & $4.22 \mathrm{E}-07$ & $4.13 \mathrm{E}-07$ & $3.76 \mathrm{E}-07$ & $3.81 \mathrm{E}-07$ & $3.78 \mathrm{E}-07$ & $1.09 \mathrm{E}-07$ & $4.23 \mathrm{E}-07$ \\
${ }^{56} \mathrm{Ni}$ & $5.86 \mathrm{E}-01$ & $6.41 \mathrm{E}-01$ & $5.64 \mathrm{E}-01$ & $6.90 \mathrm{E}-01$ & $7.73 \mathrm{E}-01$ & $5.55 \mathrm{E}-01$ & $7.37 \mathrm{E}-01$ \\
${ }^{57} \mathrm{Ni}$ & $2.27 \mathrm{E}-02$ & $1.75 \mathrm{E}-02$ & $9.95 \mathrm{E}-03$ & $1.59 \mathrm{E}-02$ & $1.98 \mathrm{E}-02$ & $9.57 \mathrm{E}-03$ & $1.74 \mathrm{E}-02$ \\
${ }^{59} \mathrm{Ni}$ & $6.71 \mathrm{E}-04$ & $6.39 \mathrm{E}-04$ & $2.54 \mathrm{E}-04$ & $2.53 \mathrm{E}-04$ & $2.51 \mathrm{E}-04$ & $2.03 \mathrm{E}-04$ & $1.90 \mathrm{E}-04$ \\
${ }^{63} \mathrm{Ni}$ & $8.00 \mathrm{E}-07$ & $7.81 \mathrm{E}-07$ & $1.69 \mathrm{E}-06$ & $1.74 \mathrm{E}-06$ & $1.73 \mathrm{E}-06$ & $1.98 \mathrm{E}-07$ & $8.31 \mathrm{E}-07$ \\
\hline
\end{tabular}

Table 5: $N_{\text {Ia }} / N_{\text {II }}$ Ratios obtained for different Models of SN Ia

\begin{tabular}{crrrrr}
\hline SN II Model & W7 & W70 & DD1 & DD2 & DD3 \\
\hline $20 \mathrm{M}_{\odot}$ & 0.214 & 0.176 & 1.08 & 0.187 & 0.146 \\
$10-50 \mathrm{M}_{\odot}$ & 0.295 & 0.243 & 1.48 & 0.257 & 0.201 \\
\hline
\end{tabular}

